Supporting Information:

\title{
EFFECTS OF A CENTRAL ATOM AND PERIPHERAL SUBSTITUENTS ON PHOTOINDUSED ELECTRON TRANSFER IN THE PHTHALOCYANINE-FULLERENE DONOR-ACCEPTOR SOLUTION-PROCESSABLE DYADS
}

\author{
Ekaterina N. Ovchenkova ${ }^{1 *}$, Natatiya G. Bichan ${ }^{1 *}$, Arshak A. Tsaturyan ${ }^{2}$, Nadezhda O. \\ Kudryakova ${ }^{1}$, Matvey S. Gruzdev ${ }^{1}$, Fedor E. Gostev ${ }^{3}$, Ivan V. Shelaev ${ }^{3}$, Victor A. Nadtochenko ${ }^{3}$, \\ Tatyana N. Lomova ${ }^{1}$ \\ ${ }^{1}$ G.A. Krestov Institute of Solution Chemistry of the Russian Academy of Sciences, \\ Akademicheskaya Str. 1, Ivanovo, Russia \\ ${ }^{2}$ Institute of Physical and Organic Chemistry, Southern Federal University, Stachki Av. 194/2 \\ Rostov-on-Don, Russia \\ ${ }^{3}$ N.N. Semenov Federal Research Center for Chemical Physics, Russian Academy of Sciences, \\ Kosygina st., 4, Moscow, Russia
}

Corresponding author : enk@isc-ras.ru, bng@isc-ras.ru

Synthesis of (octakis(3,5-di-tert-butylphenoxy)phthalocyaninato)cobalt(II), (1) ....................................... 3

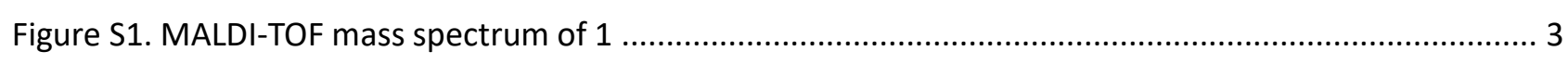

Synthesis of (Octakis(3,5-di-tert-butylphenoxy)phthalocyaninato)manganese(III) acetate, (2) ..................... 4

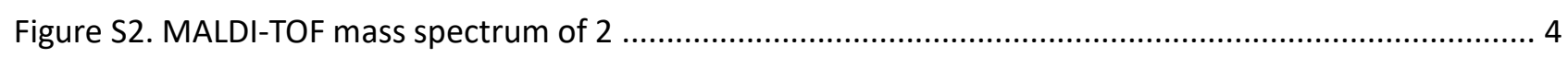

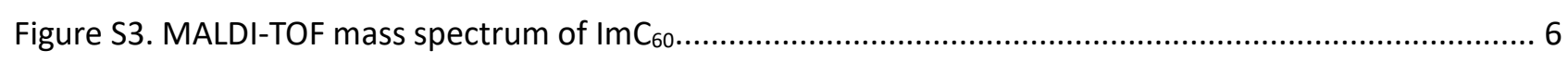

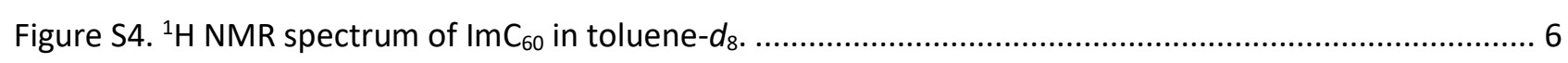

Figure S5. UV-vis spectral changes occurring during titration of 2 with imidazole $\left(C_{\mathrm{Im}}=0 \div 1.5 \times 10^{-2} \mathrm{M}\right)$ in toluene (the inset is the $\log \left(\left(A_{\mathrm{i}}-A_{0}\right) /\left(A_{\infty}-A_{\mathrm{i}}\right)\right)$ vs $\log C_{\text {Im }}$ plot used to calculate the number of Im molecules

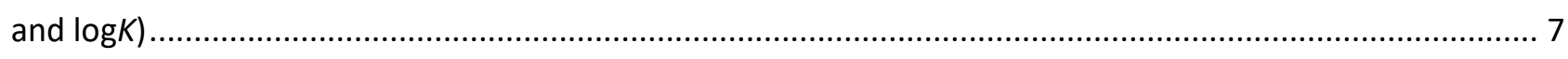

Table S1. The first-order rate constants, $\mathrm{k}_{\mathrm{obs}}$ for the reaction of 1 with $\operatorname{ImC}_{60}$ in toluene at $298 \mathrm{~K} \ldots \ldots \ldots \ldots \ldots \ldots . . . . . . .7$

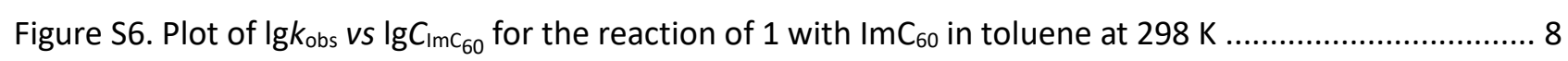

Figure S7. Visible absorption during titration of 1 with $\operatorname{ImC}_{60}\left(C_{I_{m C}}=1.43 \times 10^{-6} \div 1.74 \times 10^{-4} \mathrm{M}\right)$ in toluene at $\tau=\infty$ (in inset the corresponding spectrophotometric titration curve) (a). The plots of $\lg / \mathrm{vs} \lg C_{\operatorname{lmc}} C_{60}$ for

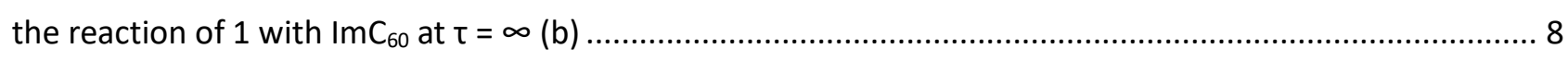

Table S2. The effective rate constants, $k_{\mathrm{obs}}$ for the reaction of 2 with $\mathrm{ImC}_{60}$ in toluene at $298 \mathrm{~K} \ldots \ldots \ldots \ldots \ldots \ldots . . . . . . . . . .8$

Figure S8. IR spectra of 1 and its dyad with $I \mathrm{ICC}_{60}$ in $\mathrm{KBr}$. The bands corresponding to vibrations of $I m C_{60}$ are denoted with asterisks

Figure S9. IR spectra of 2 and its dyad with $\mathrm{ImC}_{60}$ in $\mathrm{KBr}$. The bands corresponding to vibrations of $\mathrm{ImC} \mathrm{C}_{60}$ are denoted with asterisks 
Figure S11. Experimental (red and wine lines) and calculated (green and blue bars) UV-vis (TD-UB3LYP/6$31 \mathrm{G}$ ) spectra for compounds 1 (a) and 2 (b) in toluene.

Figure S12. Energy diagrams of the frontier $\mathrm{MO}$ (beta) levels for compounds $1,2, \operatorname{ImC}_{60}$ and its dyads in toluene calculated by using TD-UB3LYP/6-31G level of theory.

Table S3. Frontier KS orbitals compositions for the dyads.

Table S4. Selected calculated optical transitions of 1 , with oscillator strengths $(f>0.1)$, wavelengths (nm) and main orbital transition contributions (>10\%) H - HOMO, L - LUMO .

Table S5. Selected calculated optical transitions of $1: \mathrm{ImC}_{60}$, with oscillator strengths ( $\left.\mathrm{f}>0.1\right)$, wavelengths $(\mathrm{nm})$ and main orbital transition contributions (>10\%) H - HOMO, L - LUMO

Table S6. Selected calculated optical transitions of 2, with oscillator strengths $(f>0.1)$, wavelengths ( $\mathrm{nm}$ ) and main orbital transition contributions (>10\%) H - HOMO, L - LUMO .

Table S7. Selected calculated optical transitions of $2: \mathrm{ImC}_{60}$, with oscillator strengths $(\mathrm{f}>0.1)$, wavelengths $(\mathrm{nm})$ and main orbital transition contributions (>10\%) H - HOMO, L - LUMO.

Table S8. Calculated 80 electron transitions, $\wedge$ parameters values and $\Delta$-index for $1: \operatorname{ImC} C_{60}$ and $2: \operatorname{ImC}{ }_{60}$ by using B3LYP/6-31G level of theory.

Figure S13. Spin density distribution doublet states in the compounds 1 (a), $1: \operatorname{ImC} \mathrm{C}_{60}$ (c) and quintet states of 2 (b) and 2:ImC 60 (d) calculated by UB3LYP*+D3BJ/6-31G method (Cutoff at 0.01 a.u.).......

Cartesian coordinates in $\AA$ for B3LYP*+D3BJ optimized geometries

Table S9. Electrochemical redox potentials (versus the saturated calomel electrode) of investigated compounds in dichloromethane

Fig. S14. Fluorescence spectra $\left(\lambda_{\text {exc }}=395 \mathrm{~nm}\right)$ of $2\left(C_{2}=4.4 \times 10^{-6} \mathrm{~mol} \mathrm{~L}^{-1}\right), \operatorname{ImC}_{60}\left(C_{\operatorname{lmc} 60}=7.8 \times 10^{-5} \mathrm{~mol} \mathrm{~L}^{-1}\right)$ and dyad, $2: \operatorname{ImC}_{60}\left(C_{2}=4.4 \times 10^{-6} \mathrm{~mol} \mathrm{~L}^{-1}, \mathrm{C}_{\text {ImC60 }}=1.41 \times 10^{-4} \mathrm{~mol} \mathrm{~L}^{-1}\right)$.

Fig. S15. Femtosecond transient spectra of 1 at the excitation wavelength of $\lambda=690 \mathrm{~nm}$ in nitrogen saturated toluene.

Fig. S16. Femtosecond transient spectra of 2 at the excitation wavelength of $\lambda=735 \mathrm{~nm}$ in nitrogen saturated toluene. 


\section{Synthesis of (octakis(3,5-di-tert-butylphenoxy)phthalocyaninato)cobalt(II), (1)}

$\mathrm{Co}(\mathrm{AcO})_{2} \cdot 4 \mathrm{H}_{2} \mathrm{O}(42.0 \mathrm{mg}, 0.17 \mathrm{mmol})$ and (octakis(3,5-di-tert-butylphenoxy)phthalocyanine (44.0 $\mathrm{mg}, 0.02 \mathrm{mmol})$ reacted in dimethylformamide $(10 \mathrm{ml})$ at heating for $2 \mathrm{~h}$. The reaction mixture was poured into $50 \mathrm{ml}$ of methanol and the precipitate was separated by centrifugation. For purification, the product was dissolved in dichloromethane, purified by chromatography (silica $\mathrm{gel} / \mathrm{CH}_{2} \mathrm{Cl}_{2}$ ), and dried under vacuum $\left(60^{\circ} \mathrm{C}, 24 \mathrm{~h}\right)$. The individuality and the purity of the compounds were confirmed by TLC on Silufol silica gel using benzene ( $\mathrm{Rf}=0.8)$.Yield: $23 \mathrm{mg}(0.01 \mathrm{mmol}, 52 \%)$. UV-vis (toluene), $\lambda_{\max }(\log \varepsilon) \mathrm{nm}: 304$ (4.76), 340 (4.72), 607 (4.45), 646 (4.53), 673 (5.17). IR $(\mathrm{KBr}), v, \mathrm{~cm}^{-1}:$ 2964, 2905, $2868 v(\square \mathrm{C}$-H) tert-butyl groups; 1608, 1588, 1524 benzene stretching; 1457, 1415 isoindole stretching; 1363, $1348 \delta(\square \mathrm{C}$-H) tert-butyl groups; $1246 v(\mathrm{C}$-C) tert-butyl groups; 1297, 1274, 1198, 1145, 1095, 1052, 961, 903, 864, 836, 756, 726, 707, 555, 463 vibrations of macrocycles; $439 v(\mathrm{Co}-\mathrm{N}) .{ }^{1} \mathrm{H}$ NMR (toluene- $\left.d_{8}\right): 15.16(\mathrm{~s}, 8 \mathrm{H} \beta), 8.72\left(\mathrm{~s}, 16 \mathrm{H}_{\mathrm{o}}\right), 7.89\left(\mathrm{~s}, 8 \mathrm{H}_{\mathrm{p}}\right)$, 1.68 (s, 144 tert-butyl-H) ppm. MALDI-TOF MS: calc. for $\mathrm{C}_{144} \mathrm{H}_{176} \mathrm{~N}_{8} \mathrm{O}_{8} \mathrm{Co}-2203.0$, found $2205.26[\mathrm{M}+2 \mathrm{H}]^{+}$.

Figure S1. MALDI-TOF mass spectrum of 1

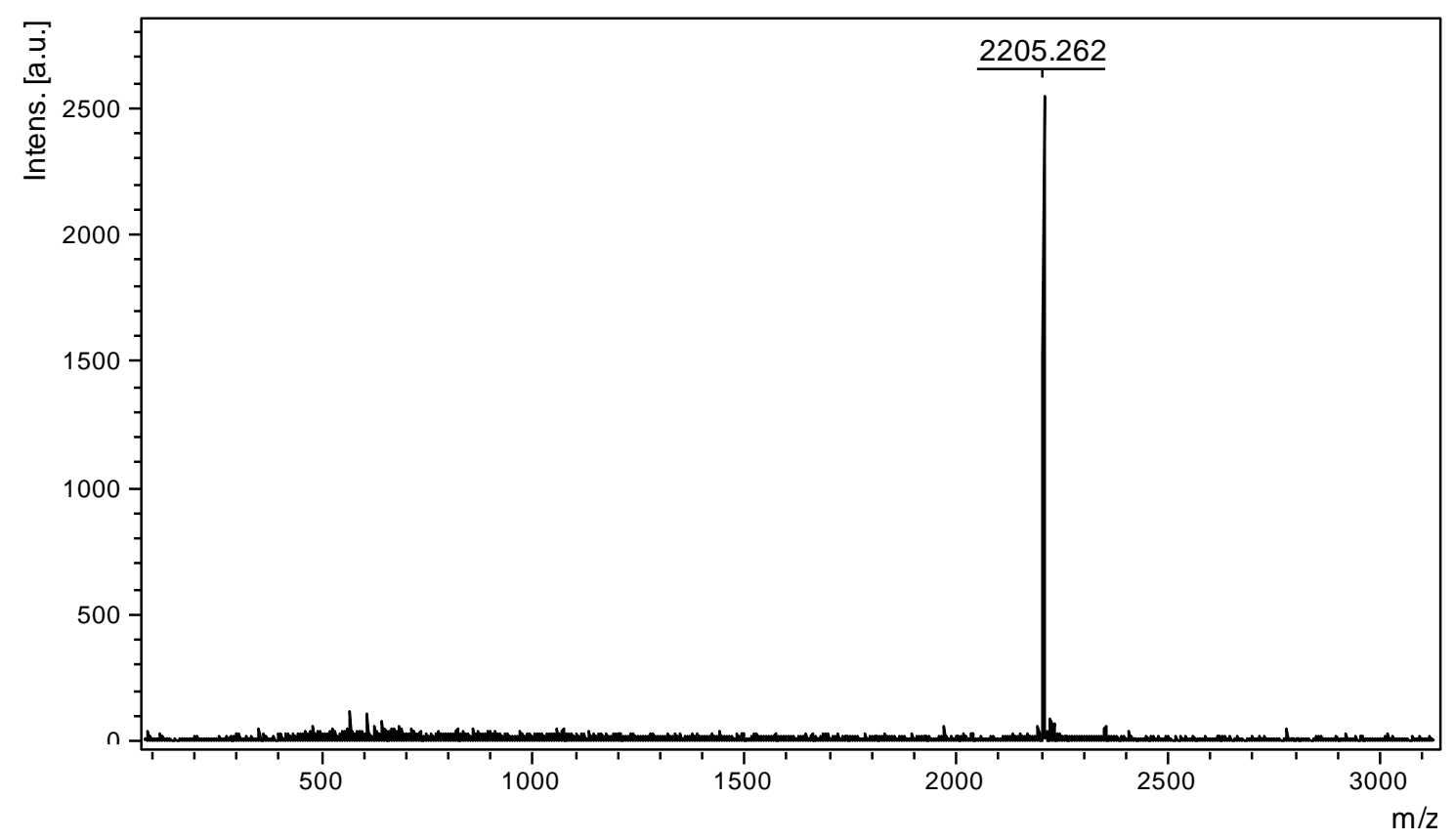


Synthesis of (Octakis(3,5-di-tert-butylphenoxy)phthalocyaninato)manganese(III) acetate, (2)

4,5-bis(3,5-di-tert-butylphenoxy)phthalonitrile $(1.25 \mathrm{~g}, 2.33 \mathrm{mmol})$ and $\mathrm{Mn}(\mathrm{AcO})_{2} \cdot 4 \mathrm{H}_{2} \mathrm{O}(0.25 \mathrm{~g}$,

$1.02 \mathrm{mmol})$ reacted in hexanol-1 $(6 \mathrm{ml})$ under reflux in the presence of DBU (6 drops) at $160^{\circ} \mathrm{C}$. The reaction mixture was poured into $60 \mathrm{ml}$ of methanol, precipitated by drop wise addition of water, and filtered. The obtained precipitate was dissolved in chloroform and chromatographed (silica gel/ $\left.\mathrm{CHCl}_{3}-1 \% \mathrm{CH}_{3} \mathrm{OH}\right)$. Yield: $0.61 \mathrm{~g}(0.27 \mathrm{mmol}, 46 \%)$. UV-vis (toluene), $\lambda_{\max }(\log \varepsilon) \mathrm{nm}$ : 407 (4.56), 655 (4.27), 727 (5.03). IR spectrum (KBr), v, cm ${ }^{-1}:$ 2963, 2928, 2868, 1725, 1609, 1586, 1508, 1459, 1421, 1407, 1384, 1363, 1337, 1296, 1246, 1199, 1119, 1084, 1042, 1002, 961, 903, 864, 745, 707. IR spectrum (CsBr), $v, \mathrm{~cm}^{-1}: 650,554,530,503,439,305 v(\mathrm{Mn}-\mathrm{N}) .{ }^{1} \mathrm{H}$ NMR $\left(\mathrm{CDCl}_{3}\right):{ }^{1} \mathrm{H} \mathrm{NMR}(\delta, \mathrm{ppm} ; \mathrm{CDCl} 3): 7.05$ (br. s), 6.97 (s), 6.74 (br. s), 2.19 (s, tert-butyl-H). MALDI-TOF MS: calc. for $\mathrm{C}_{144} \mathrm{H}_{176} \mathrm{~N}_{8} \mathrm{O}_{8} \mathrm{Mn}-2199.0$, found 2199.52 [M-AcO] $]^{+}$.

Figure S2. MALDI-TOF mass spectrum of 2

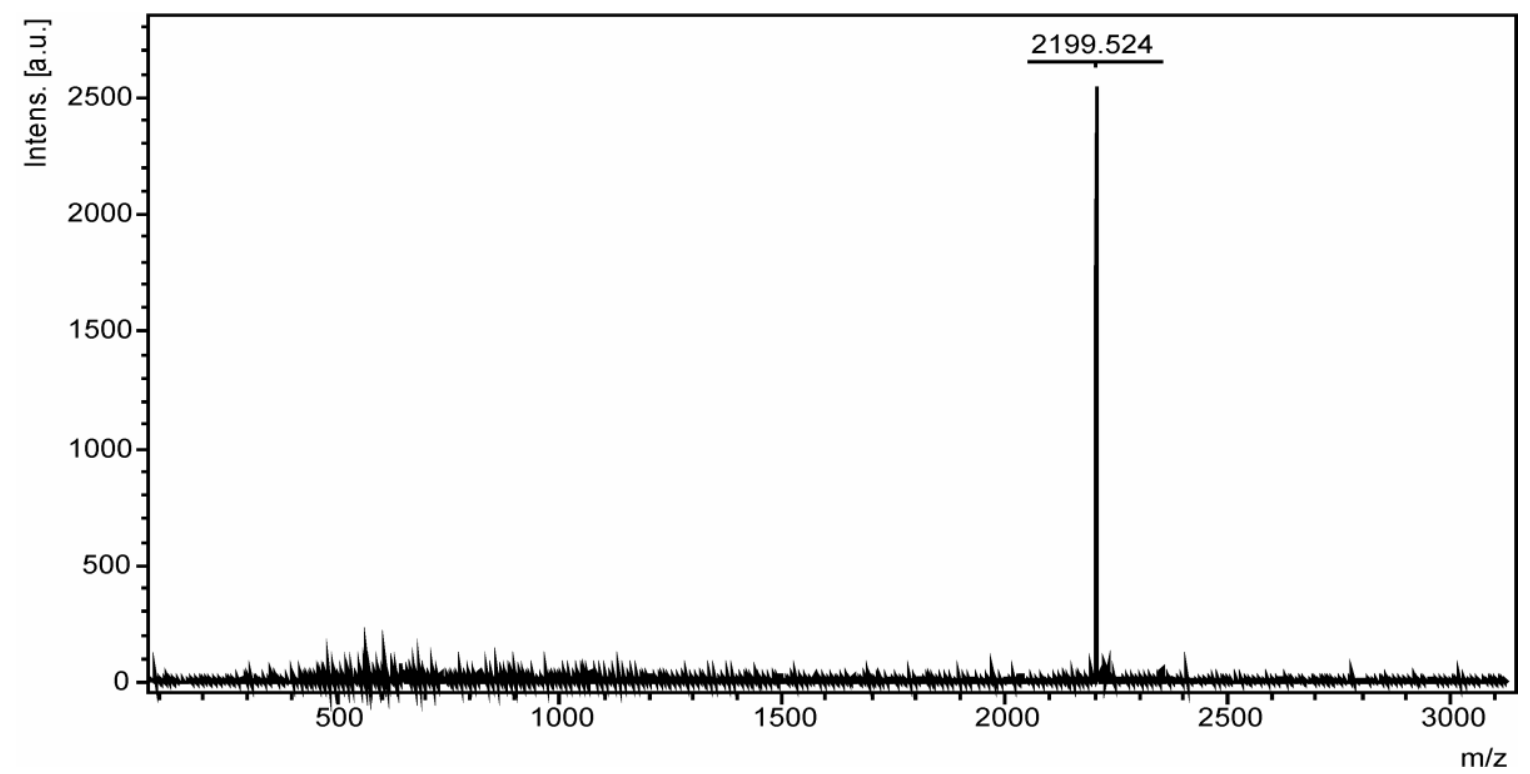




\section{Synthesis of $\quad 1^{\prime}$-N-methyl-2'-(1H-imidazol-1-yl)-phenylpyrrolidino[3',4':1,2][60]fullerene,}

ImC60 was prepared according to a general procedure of fulleropyrrolidine synthesis developed by Prato and coworkers [Prato M., Maggini M., Giacometti C., Scorrano G., Sandona G., Farnia G. Synthesis and electrochemical properties of substituted fulleropyrrolidines. Tetrahedron. 1996;52:5221-5234]. The some changes (the ratio of reagents, the reaction time, the purification method) were made to the procedure, which led to an increase in the yield of the product. A solution of $100 \mathrm{mg}(0.14 \mathrm{mmol})$ of $\mathrm{C}_{60}, 120 \mathrm{mg}(0.70 \mathrm{mmol})$ of 4-(1H-imidazol-1-yl) benzaldehyde and 40 $\mathrm{mg}(0.45 \mathrm{mmol})$ of $\mathrm{N}$-methylglycine in $90 \mathrm{ml}$ of toluene was stirred at reflux temperature for $2 \mathrm{~h}$. Then the solvent was removed in vacuum. The reaction mixture was purified over $\mathrm{Al}_{2} \mathrm{O}_{3}$ column using toluene (for the first zone containing unreacted $\mathrm{C}_{60}$ ), methanol (for the second zone containing unreacted 4-(1H-imidazol-1-yl) benzaldehyde) and toluene-ethyl acetate (4:1) (for the third zone containing $\left.\mathrm{ImC}_{60}\right)$ as eluent. Yield: $0.65 \mathrm{mg}(51 \%)$. UV-vis (toluene), $\lambda_{\max }(\log \varepsilon) \mathrm{nm}$ : 434 (3.54). IR spectrum (KBr), v, cm ${ }^{-1}: 3114,2946,2919,2839,2779,2346,2328,1610,1540$, $1521,1483,1463,1447,1429,1367,1357,1333,1320,1301,1282,1259,1243,1233,1215,1179$, 1163, 1151, 1143, 1107, 1099, 1091, 1053, 1033, 1018, 962, 908, 901, 863, 842, 833, 802, 789, 771, 767, 759, 746, 737, 727, 706, 694, 656, 617, 606, 599, 583, 575, 566, 562, 553, 544, 527, 476, 464, 430, 399, 376. ${ }^{1} \mathrm{H}$ NMR (toluene- $\left.d_{8}\right)$ : 7.60 (br.s, 1H), 7.44 (s, 1H), 7.18 (s, 1H), 7.08 (s, 1H), $6.82(\mathrm{~d}, J=8.03 \mathrm{~Hz}, 2 \mathrm{H}), 6.68(\mathrm{~s}, 1 \mathrm{H}), 4.60(\mathrm{~s}, 1 \mathrm{H}), 4.48(\mathrm{~d}, J=9.21 \mathrm{~Hz}, 1 \mathrm{H}), 3.80(\mathrm{~d}, J=9.43$ $\mathrm{Hz}, 1 \mathrm{H}), 2.49$ (s, 3H) ppm. MALDI-TOF MS: calc. for $\mathrm{C}_{72} \mathrm{H}_{13} \mathrm{~N}_{3}-920.0$, found $920.75[\mathrm{M}]^{+}$. 
Figure S3. MALDI-TOF mass spectrum of ImC 60

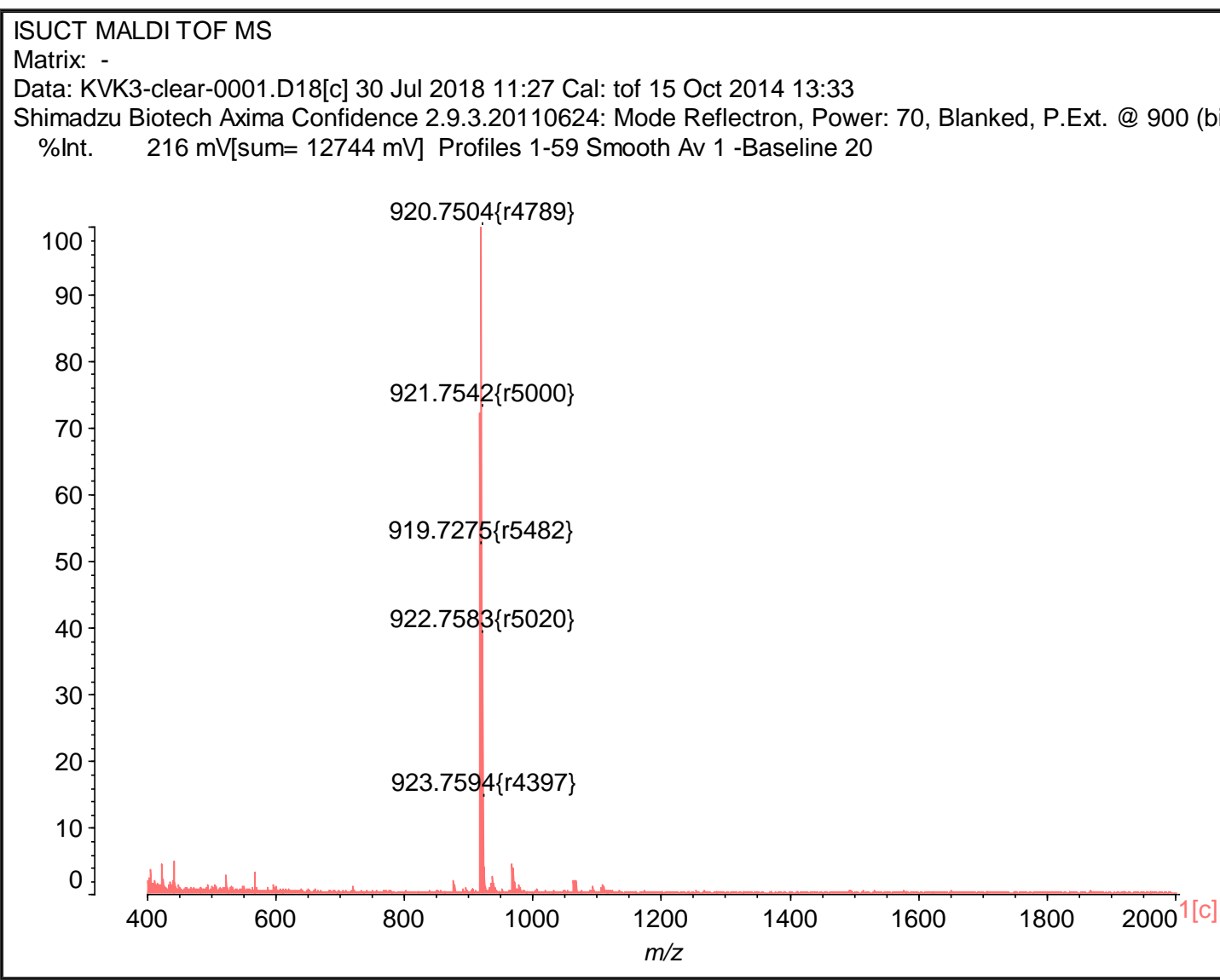

Figure S4. ${ }^{1} \mathrm{H}$ NMR spectrum of $\operatorname{ImC}_{60}$ in toluene-d8.

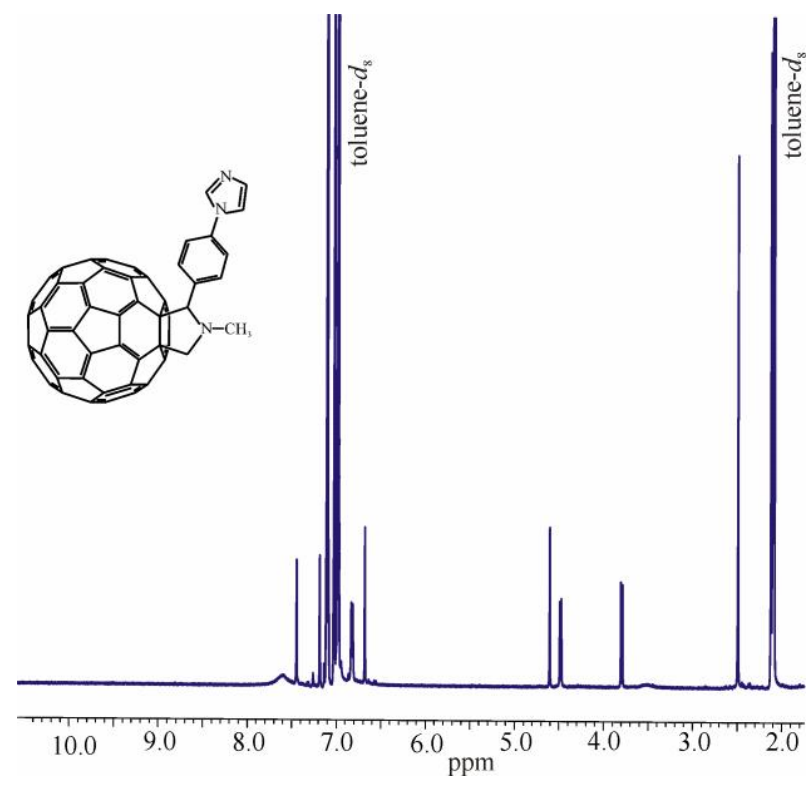


Figure S5. UV-vis spectral changes occurring during titration of 2 with imidazole $\left(C_{\operatorname{Im}}=0 \div\right.$ $\left.1.5 \times 10^{-2} \mathrm{M}\right)$ in toluene (the inset is the $\log \left(\left(A_{\mathrm{i}}-A_{0}\right) /\left(A_{\infty}-A_{\mathrm{i}}\right)\right)$ vs $\log C_{\mathrm{Im}}$ plot used to calculate the number of $\operatorname{Im}$ molecules and $\log K$ )

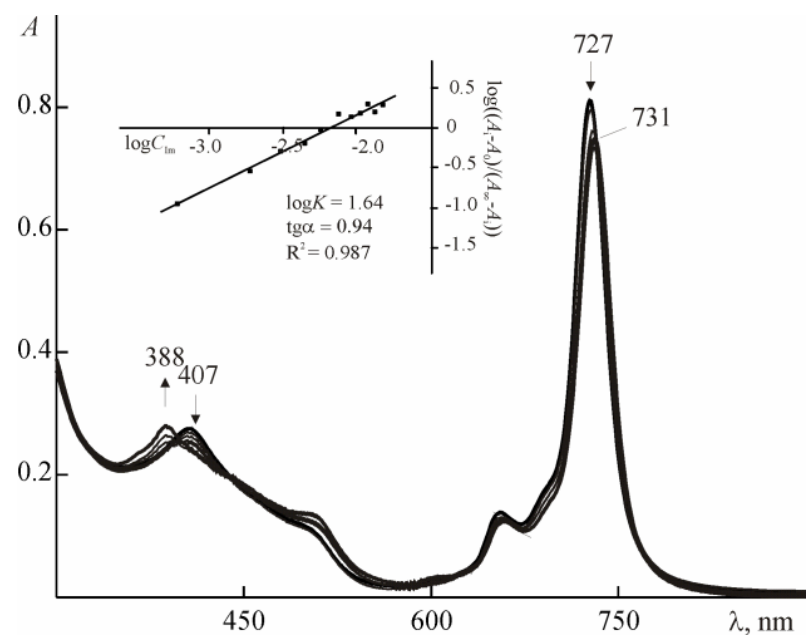

Table S1. The first-order rate constants, $k_{o b s}$ for the reaction of 1 with $\operatorname{ImC}_{60}$ in toluene at 298 K.

\begin{tabular}{l|l}
\hline$C_{\mathrm{ImC}_{60}} \times 10^{5} / \mathrm{M}$ & $\left(k_{\mathrm{obs}} \pm \delta k_{\mathrm{obs}}\right) \times 10^{5} / \mathrm{s}^{-1}$ \\
\hline 2.04 & $2.90 \pm 0.2$ \\
3.07 & $5.03 \pm 0.3$ \\
5.11 & $8.88 \pm 0.3$ \\
7.15 & $14.4 \pm 0.1$ \\
9.2 & $16.7 \pm 1.1$ \\
11.2 & $22.1 \pm 1.2$ \\
14.3 & $34.5 \pm 2.9$ \\
\hline
\end{tabular}


Figure S6. Plot of $\lg k_{0 b s} v s \lg C_{\operatorname{ImC}_{60}}$ for the reaction of 1 with $\operatorname{ImC} C_{60}$ in toluene at $298 \mathrm{~K}$

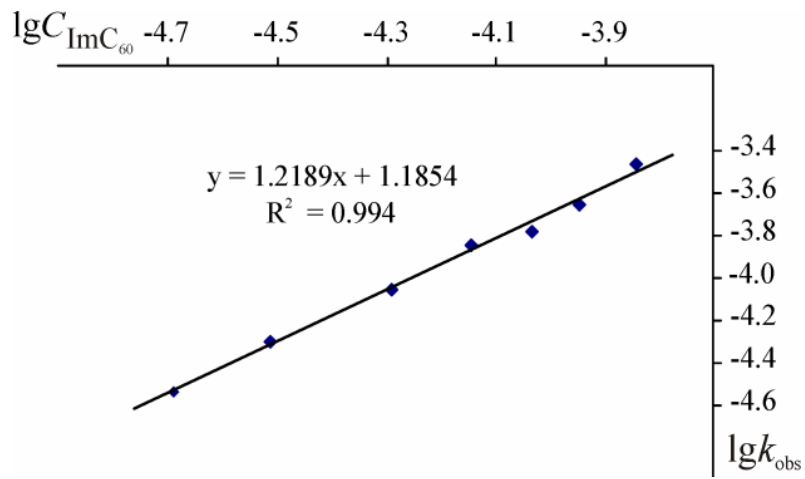

Figure S7. Visible absorption during titration of 1 with $\operatorname{ImC}_{60}\left(C_{\mathrm{ImC}_{60}}=1.43 \times 10^{-6} \div 1.74 \times 10^{-}\right.$ ${ }^{4} \mathrm{M}$ ) in toluene at $\tau=\infty$ (in inset the corresponding spectrophotometric titration curve) (a). The plots of $\lg I \mathrm{vs} \lg C_{\operatorname{ImC}_{60}}$ for the reaction of 1 with $\operatorname{ImC} \mathrm{C}_{60}$ at $\tau=\infty$ (b)
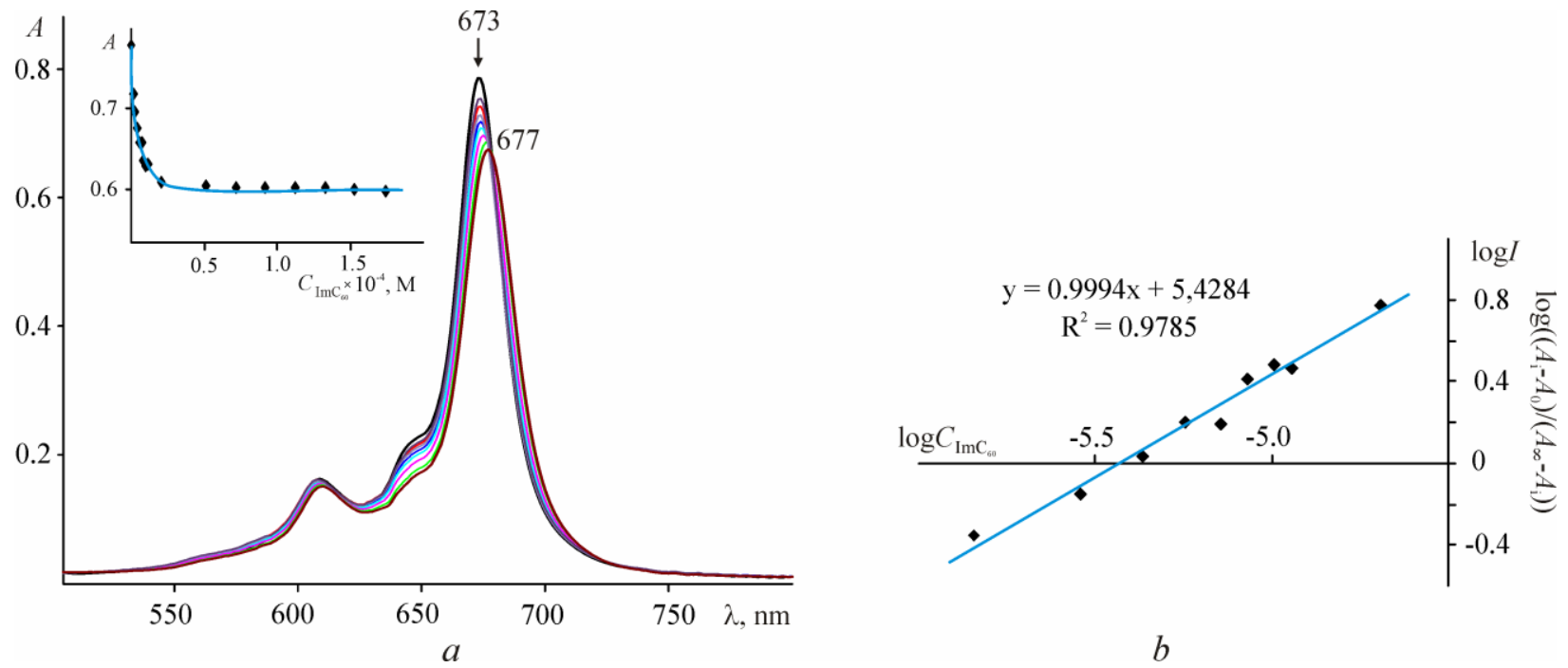

Table S2. The effective rate constants, $k_{\text {obs }}$ for the reaction of 2 with $\mathrm{ImC}_{60}$ in toluene at $298 \mathrm{~K}$ 


\begin{tabular}{cc}
\hline$C_{\mathrm{ImC}_{60}} \cdot 10^{5} / \mathrm{M}$ & $\left(k_{\mathrm{obs}} \pm \delta k_{\mathrm{obs}}\right) \times 10^{3}, \mathrm{~s}^{-1}$ \\
\hline 5.64 & $5.11 \pm 0.42$ \\
6.44 & $5.52 \pm 0.32$ \\
7.52 & $5.72 \pm 0.45$ \\
8.46 & $4.98 \pm 0.45$ \\
10.3 & $5.52 \pm 0.49$ \\
12.2 & $4.94 \pm 0.37$ \\
14.1 & $6.13 \pm 0.46$ \\
\hline
\end{tabular}

Figure S8. IR spectra of 1 and its dyad with $\mathrm{ImC}_{60}$ in $\mathrm{KBr}$. The bands corresponding to vibrations of $\mathrm{ImC}_{60}$ are denoted with asterisks

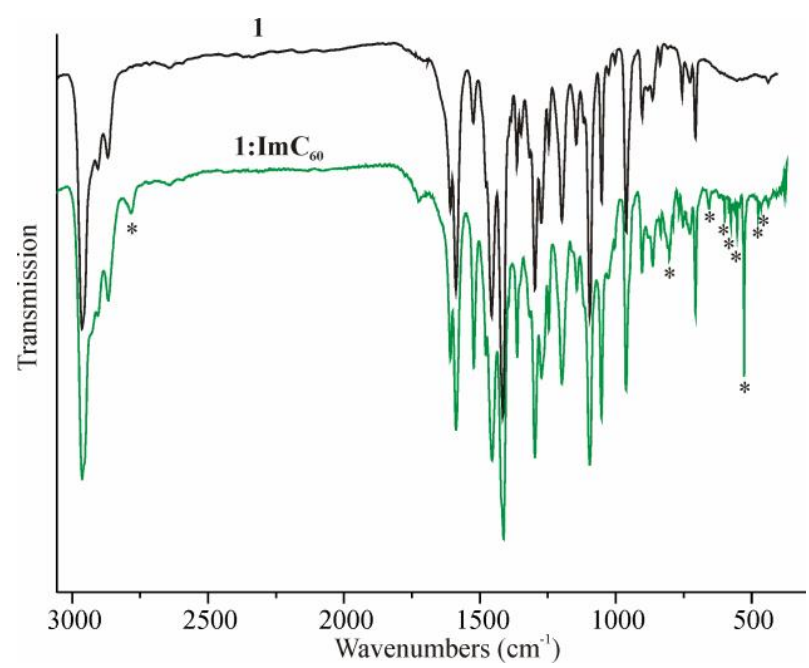

Figure S9. IR spectra of 2 and its dyad with $\mathrm{ImC}_{60}$ in $\mathrm{KBr}$. The bands corresponding to vibrations of $\mathrm{ImC}_{60}$ are denoted with asterisks

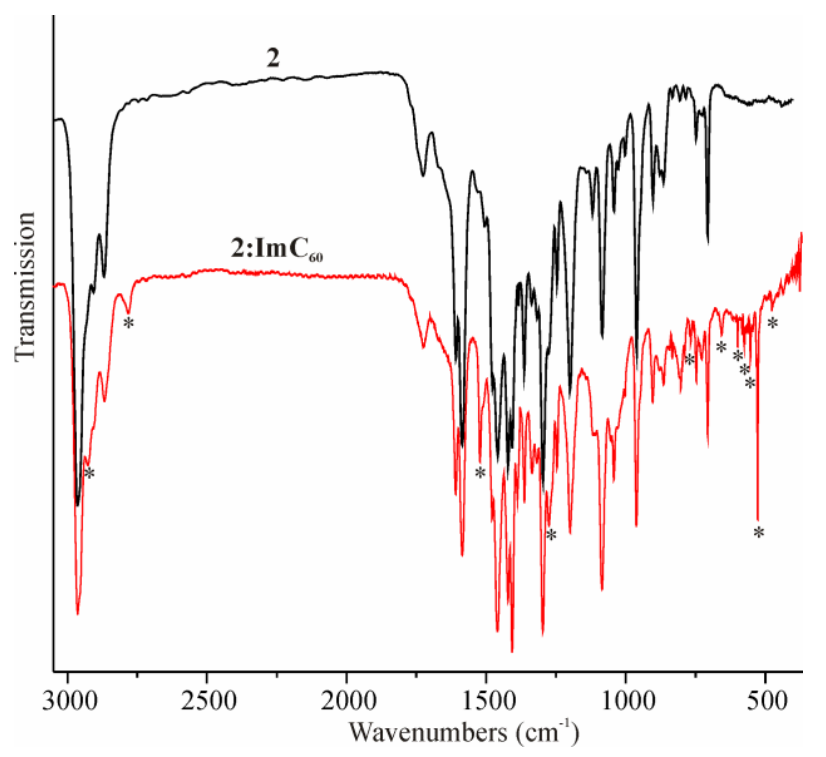


Figure S10. ${ }^{1} \mathrm{H}$ NMR spectrum of 2 (red), 2:ImC 60 (black) and $\operatorname{ImC}_{60}$ (blue) in $\mathrm{CDCl}_{3}$.

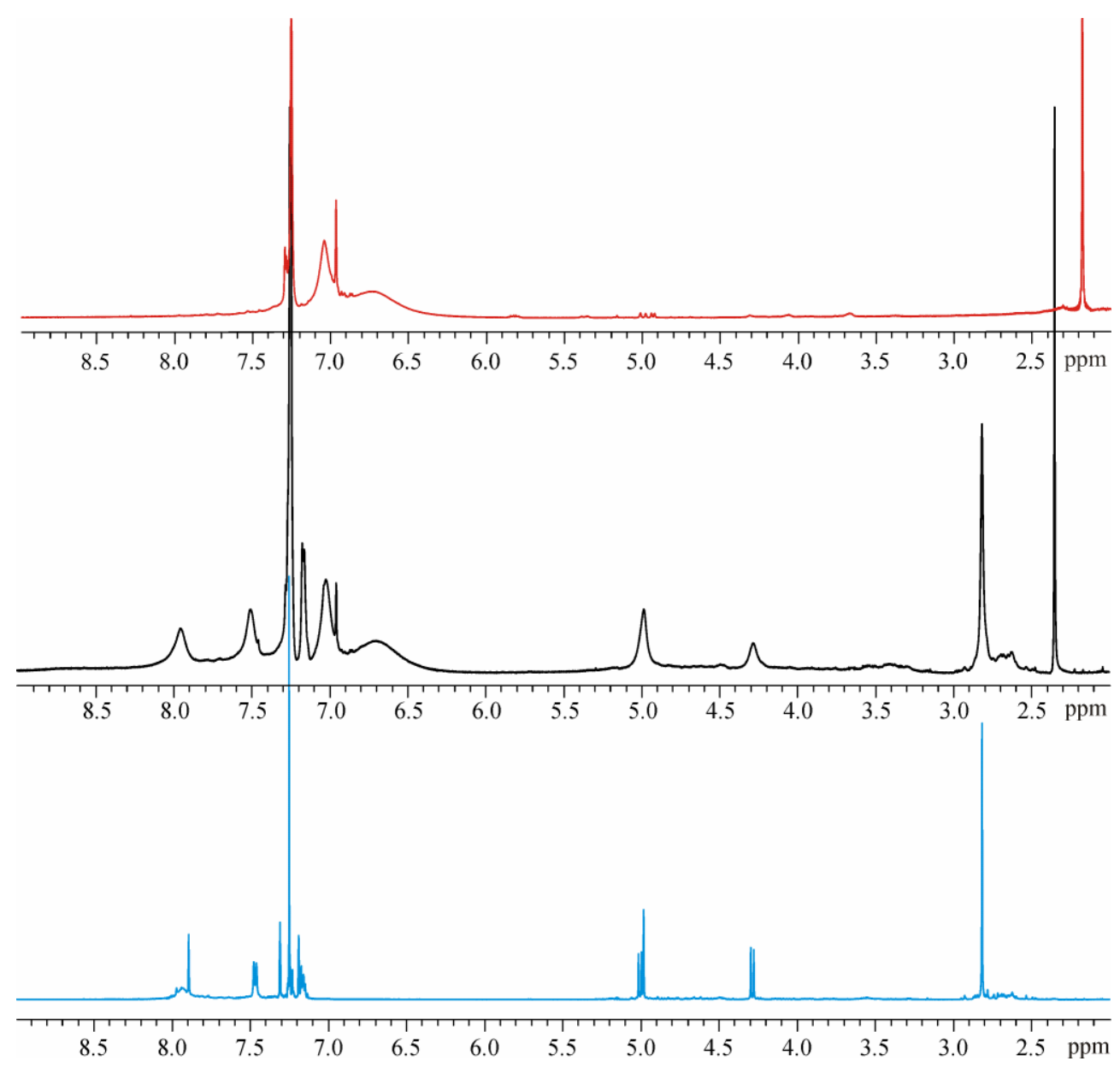

Figure S11. Experimental (red and wine lines) and calculated (green and blue bars) UV-vis (TD-UB3LYP/6-31G) spectra for compounds 1 (a) and 2 (b) in toluene.
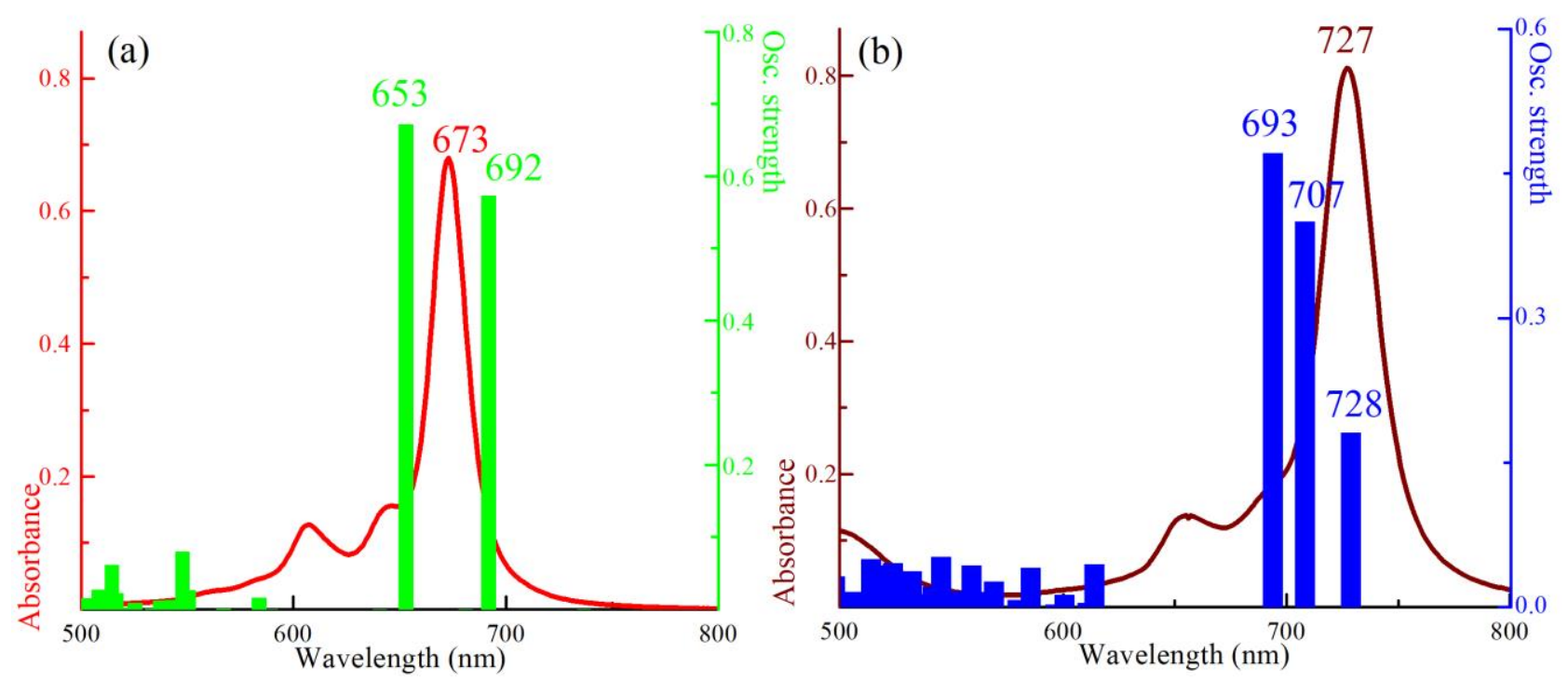
Figure S12. Energy diagrams of the frontier MO (beta) levels for compounds 1, 2, ImC 60 and its dyads in toluene calculated by using TD-UB3LYP/6-31G level of theory.

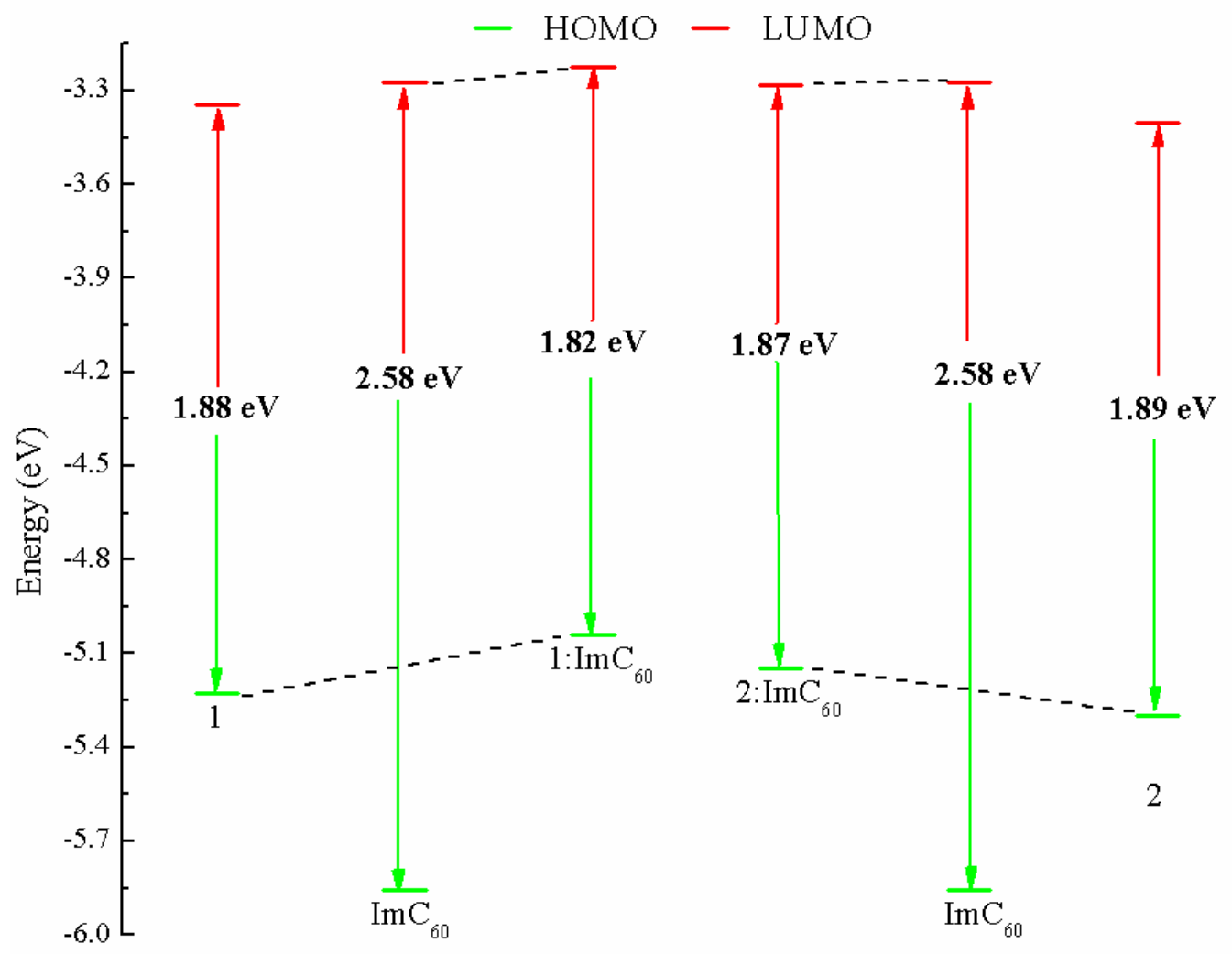

Table S3. Frontier KS orbitals compositions for the dyads.

\begin{tabular}{|c|c|c|c|c|c|c|c|}
\hline \multicolumn{8}{|c|}{$\alpha-$ orbitals } \\
\hline $\mathrm{MO}$ & $\mathrm{Co}$ & $\mathrm{Pc}$ & $\operatorname{ImC}_{60}$ & $\mathrm{Mn}$ & Pc & $\operatorname{ImC}_{60}$ & $\mathrm{AcO}$ \\
\hline LUMO+4 & 4 & 95 & 1 & 0 & 4 & 96 & 0 \\
\hline LUMO+3 & 5 & 94 & 1 & 2 & 97 & 1 & 0 \\
\hline LUMO+2 & 0 & 2 & 98 & 2 & 95 & 3 & 0 \\
\hline LUMO+1 & 0 & 1 & 99 & 0 & 2 & 98 & 0 \\
\hline LUMO & 0 & 1 & 99 & 0 & 1 & 99 & 0 \\
\hline HOMO & 0 & 99 & 1 & 42 & 13 & 13 & 32 \\
\hline HOMO-1 & 25 & 71 & 4 & 1 & 96 & 1 & 2 \\
\hline HOMO-2 & 35 & 55 & 10 & 3 & 12 & 1 & 84 \\
\hline HOMO-3 & 41 & 45 & 14 & 0 & 96 & 1 & 3 \\
\hline HOMO-4 & 9 & 84 & 7 & 0 & 54 & 46 & 0 \\
\hline \multicolumn{8}{|c|}{$\beta$ - orbitals } \\
\hline MO & $\mathrm{Co}$ & $\mathrm{Pc}$ & $\operatorname{ImC}_{60}$ & $\mathrm{Mn}$ & Pc & $\mathrm{ImC}_{60}$ & $\mathrm{AcO}$ \\
\hline LUMO+4 & 7 & 92 & 1 & 0 & 2 & 98 & 0 \\
\hline LUMO+3 & 8 & 90 & 2 & 4 & 78 & 16 & 2 \\
\hline LUMO+2 & 0 & 2 & 98 & 1 & 13 & 86 & 0 \\
\hline LUMO+1 & 0 & 1 & 99 & 6 & 89 & 4 & 1 \\
\hline LUMO & 0 & 1 & 99 & 0 & 4 & 96 & 0 \\
\hline
\end{tabular}




\begin{tabular}{|l|l|l|l|l|l|l|l|}
\hline HOMO & 0 & 99 & 1 & 0 & 98 & 0 & 2 \\
\hline HOMO-1 & 60 & 38 & 2 & 2 & 12 & 0 & 86 \\
\hline HOMO-2 & 52 & 41 & 7 & 0 & 99 & 0 & 1 \\
\hline HOMO-3 & 1 & 96 & 3 & 0 & 52 & 48 & 0 \\
\hline HOMO-4 & 1 & 96 & 3 & 0 & 87 & 12 & 1 \\
\hline
\end{tabular}

Table S4. Selected calculated optical transitions of 1 , with oscillator strengths $(f>0.1)$, wavelengths $(\mathrm{nm})$ and main orbital transition contributions (>10\%) H - HOMO, L - LUMO

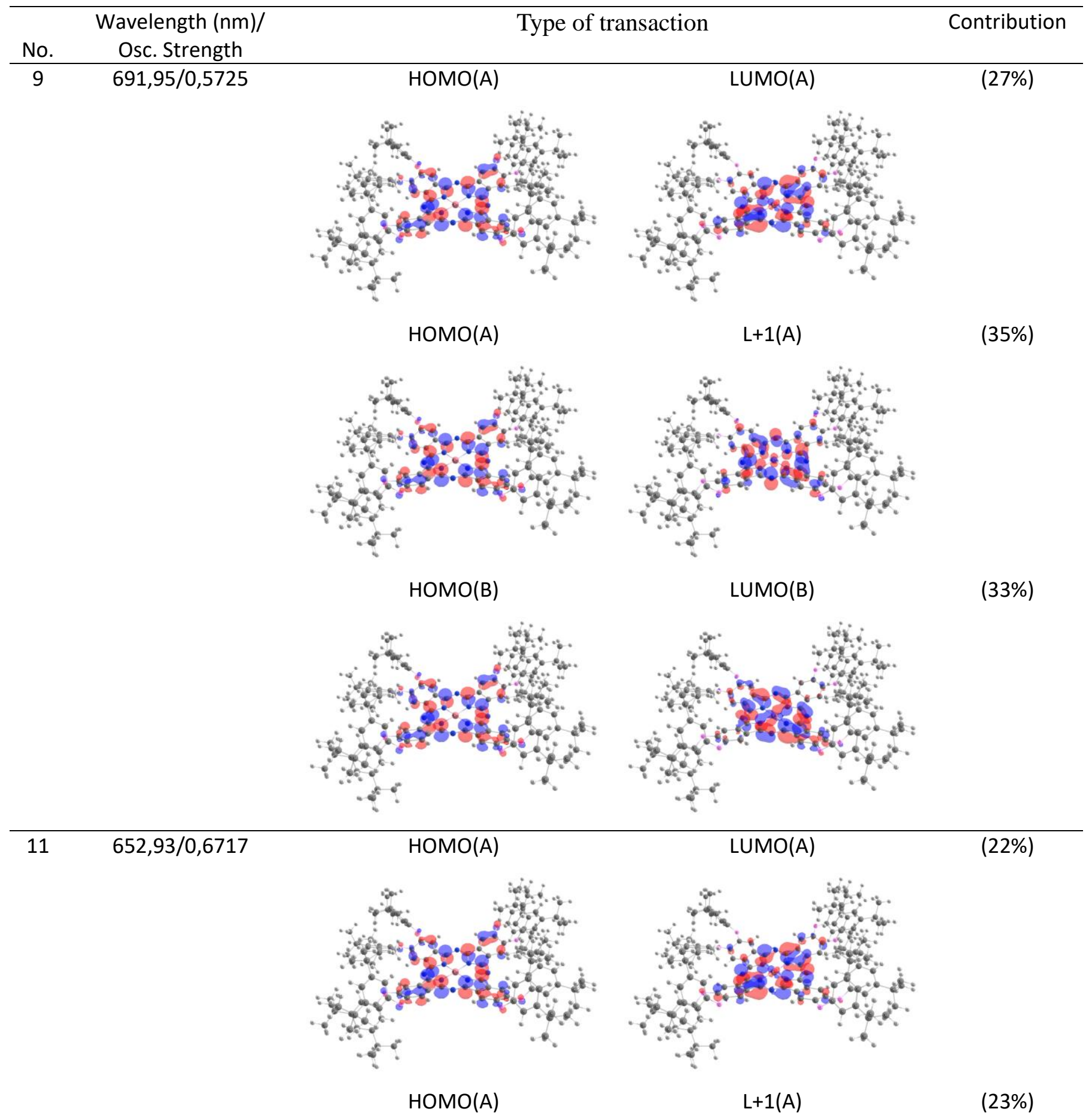




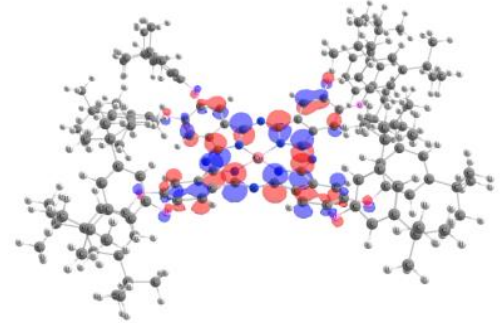

HOMO(B)

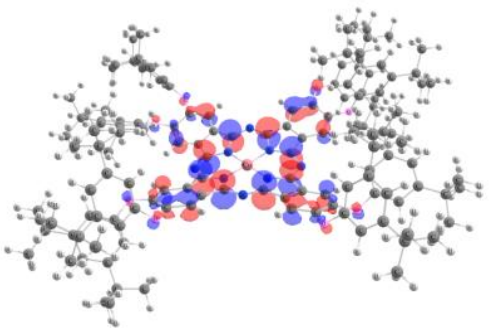

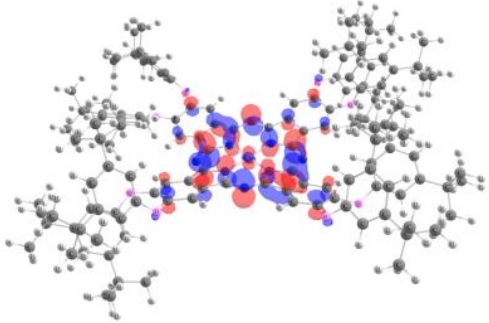

$L+1(B)$

(49\%)

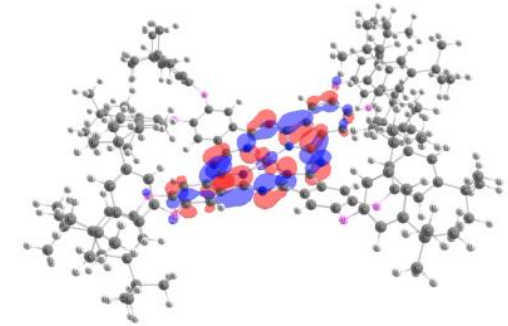

Table S5. Selected calculated optical transitions of 1:ImC60, with oscillator strengths (f > 0.1), wavelengths $(\mathrm{nm})$ and main orbital transition contributions (>10\%) H - HOMO, L - LUMO

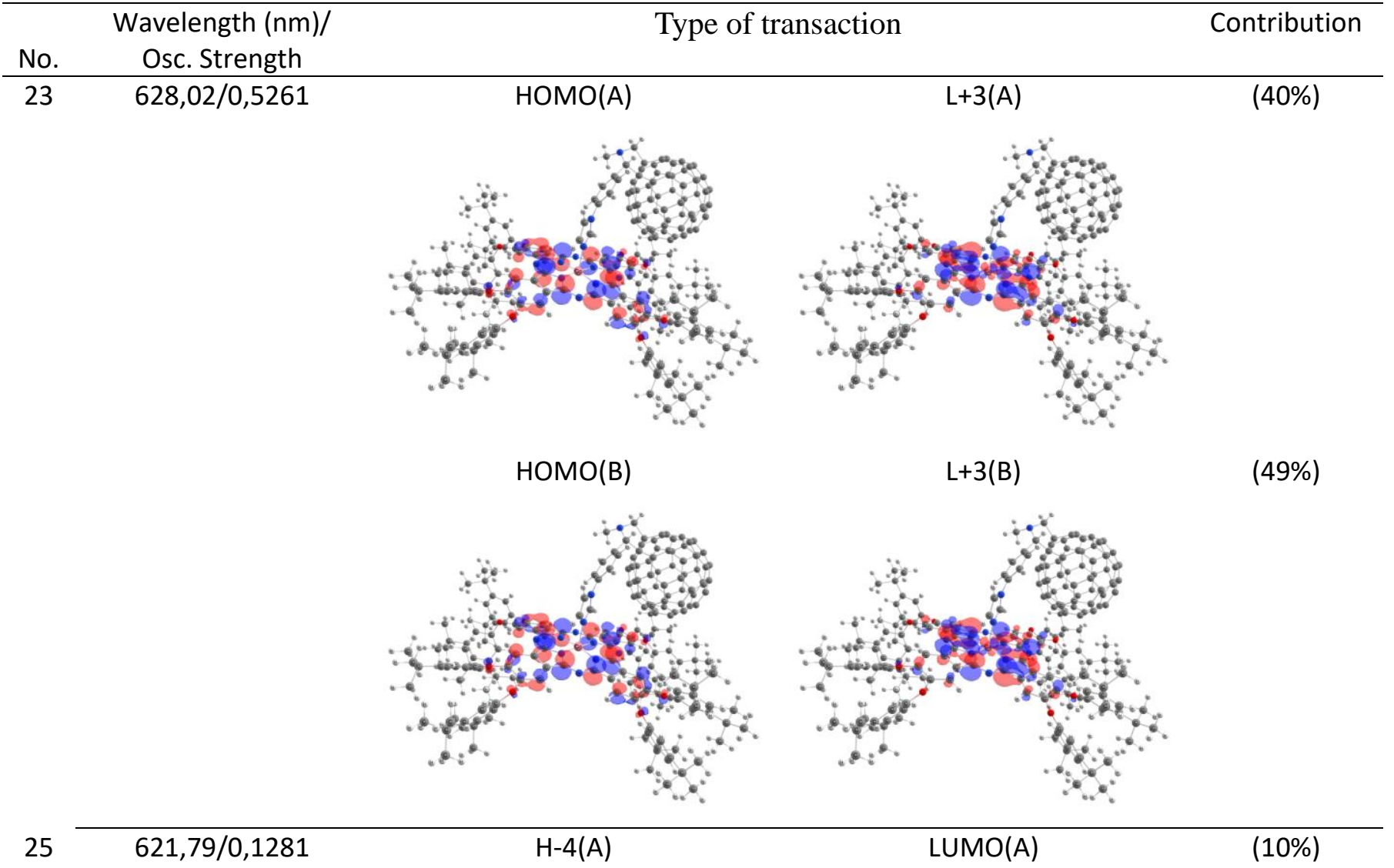




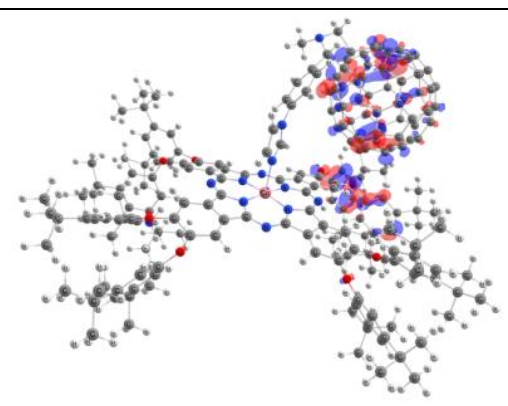

H-4(B)

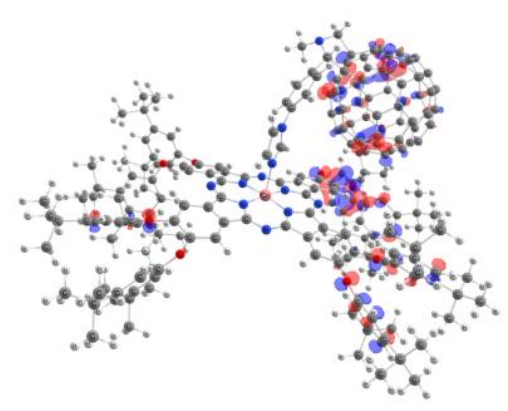

H-1(B)

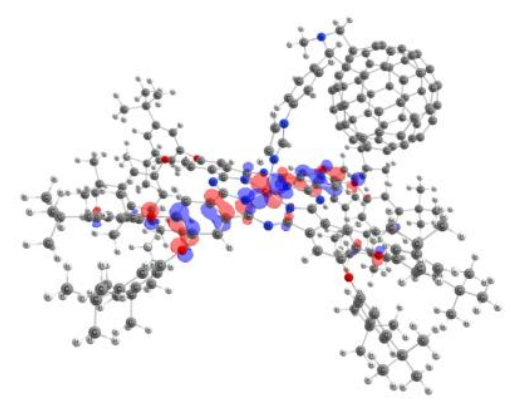

HOMO(A)

26

$620,04 / 0,6465$

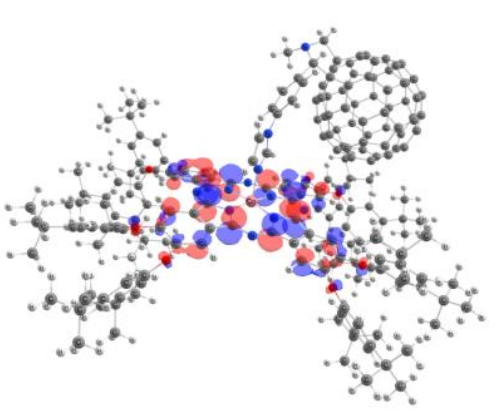

$$
\text { HOMO(B) }
$$

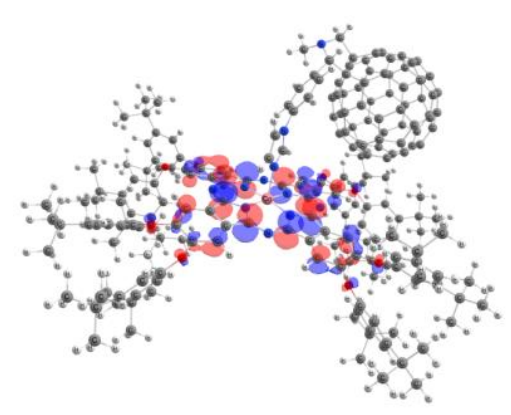

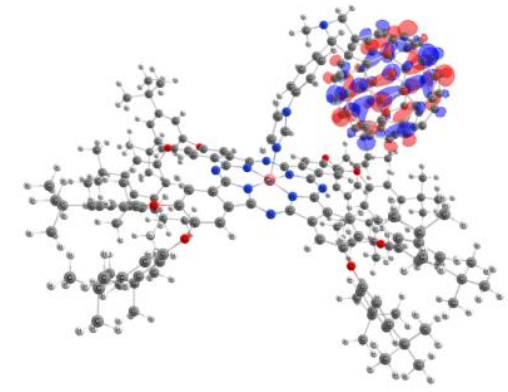

LUMO(B)

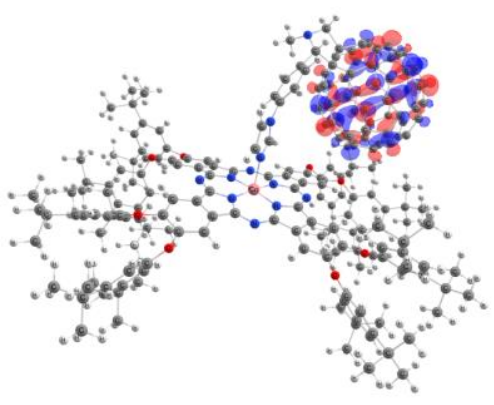

LUMO(B)

(38\%)

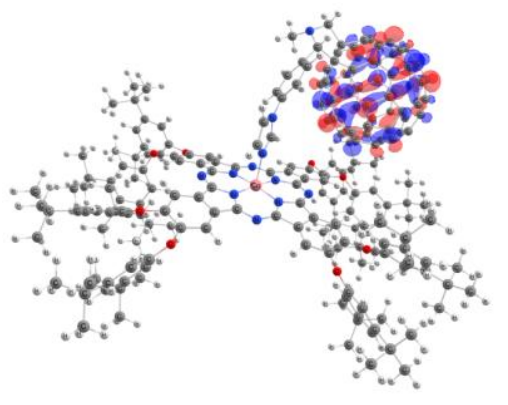

$\mathrm{L}+4(\mathrm{~A})$

(33\%)

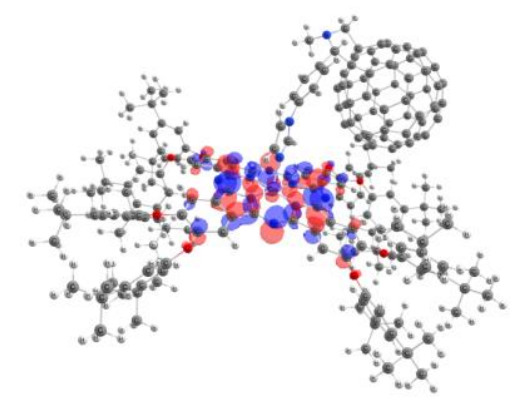

$L+4(B)$

(41\%)

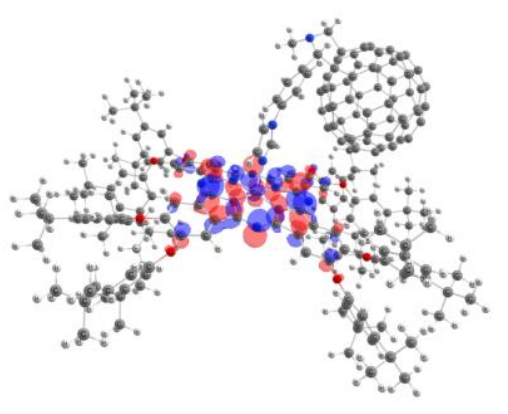


Table S6. Selected calculated optical transitions of 2, with oscillator strengths (f $>0.1$, wavelengths (nm) and main orbital transition contributions (>10\%) H - HOMO, L - LUMO

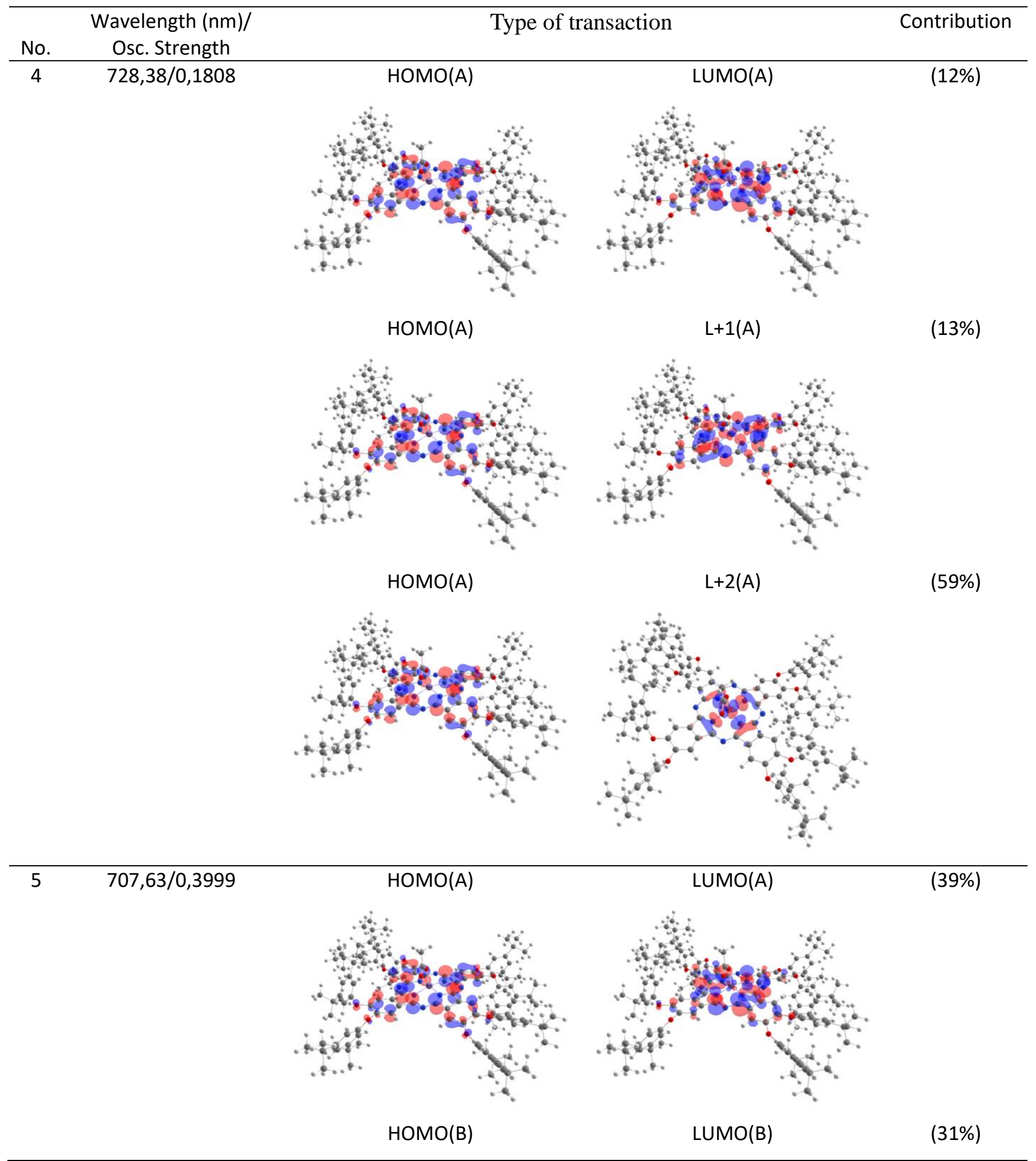



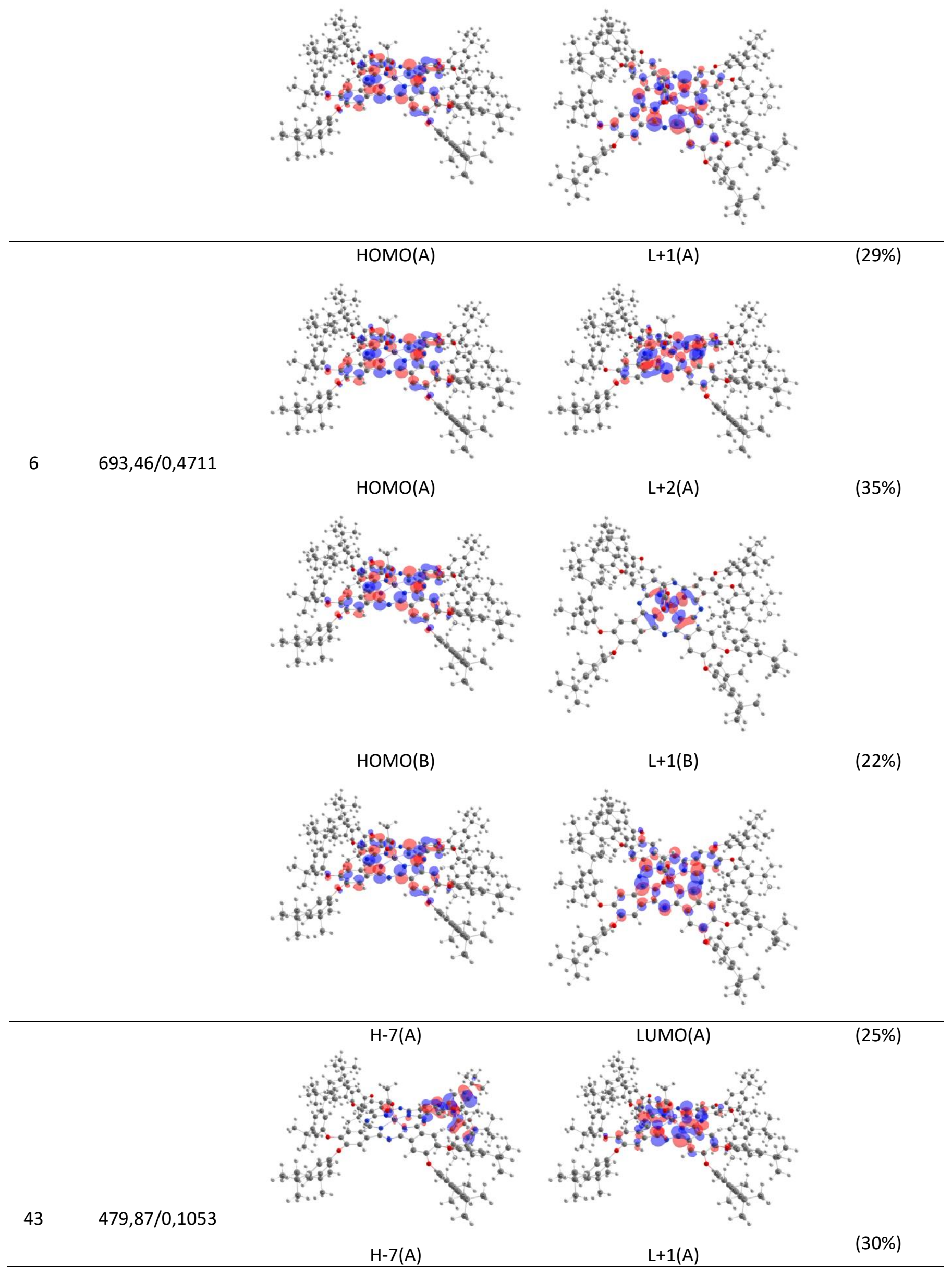

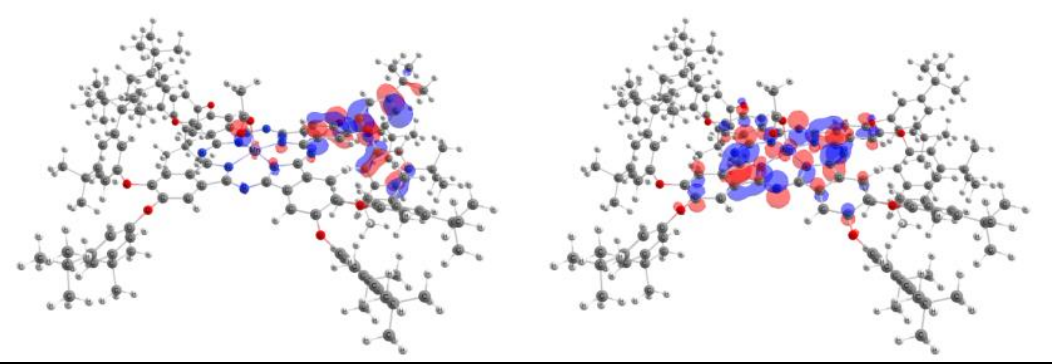

Table S7. Selected calculated optical transitions of 2:ImC60, with oscillator strengths (f > 0.1), wavelengths $(\mathrm{nm})$ and main orbital transition contributions $(>10 \%)$ H - HOMO, L - LUMO

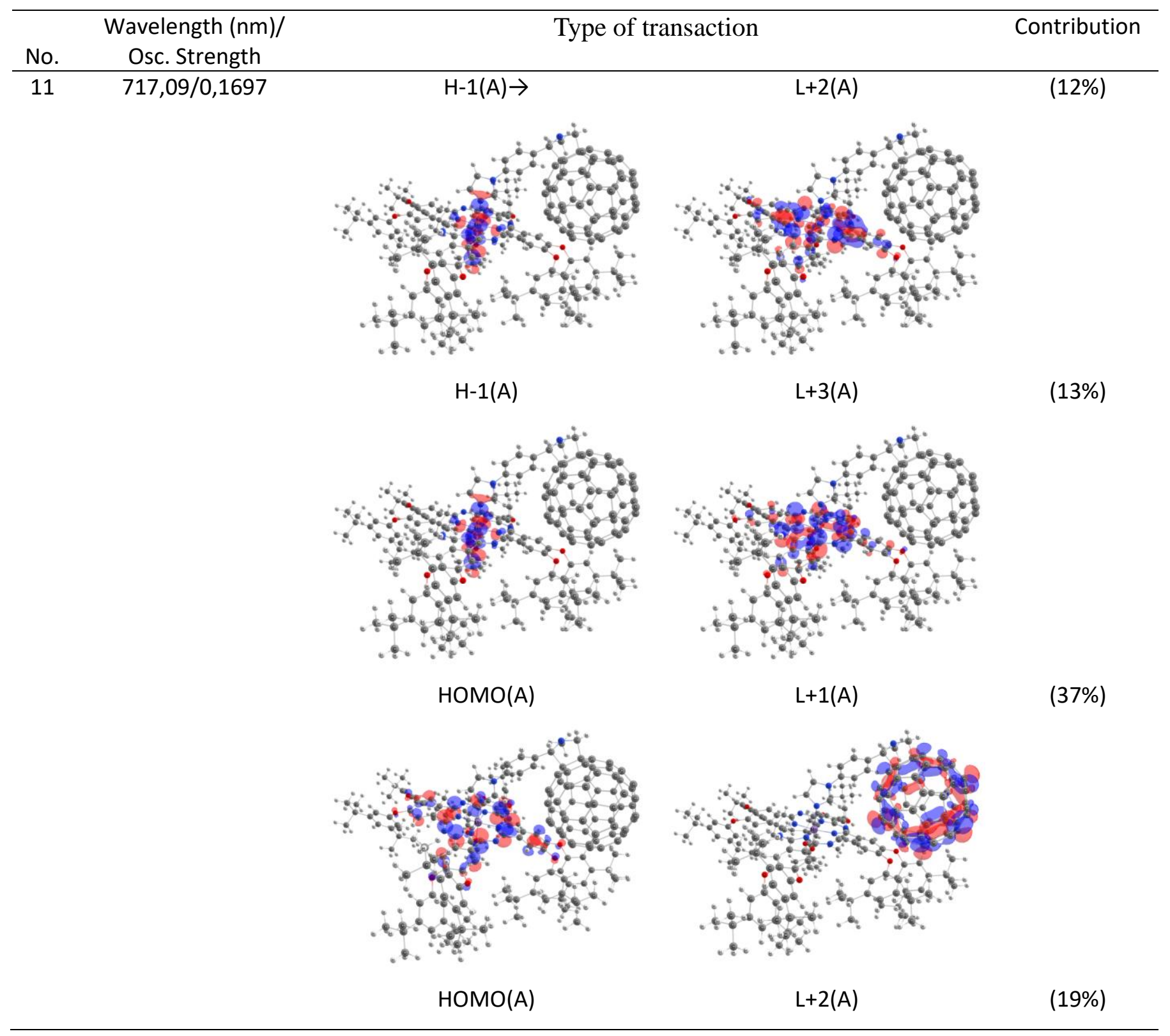



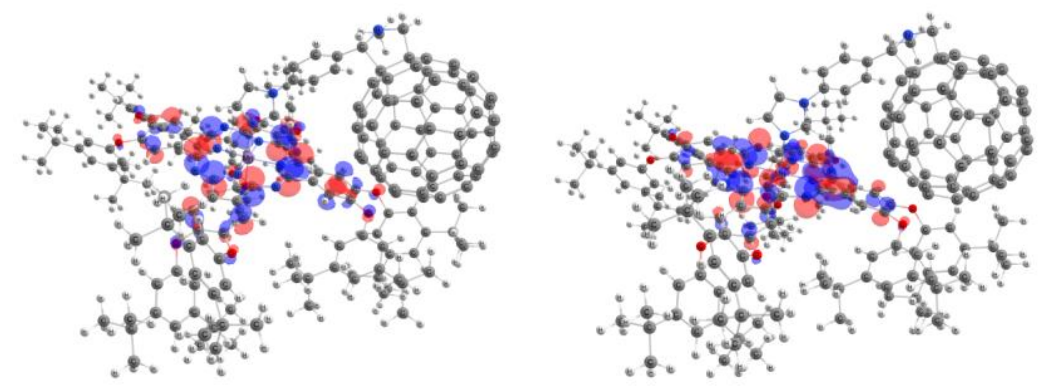

$11 \quad 715,43 / 0,0987$

$H-1(A)$

$\mathrm{L}+3(\mathrm{~A})$

(12\%)

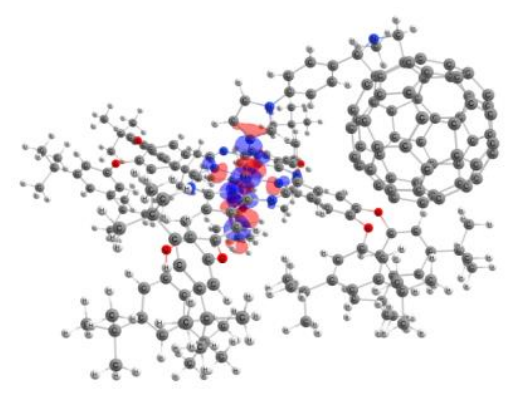

HOMO(A)

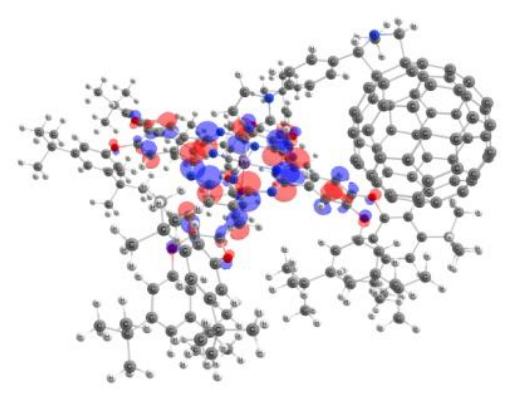

HOMO(A)

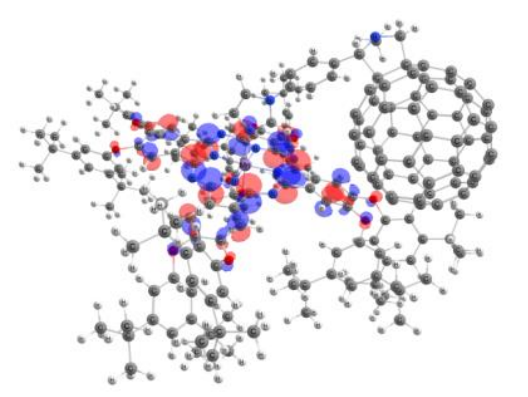

$\mathrm{H}-1(\mathrm{~A})$

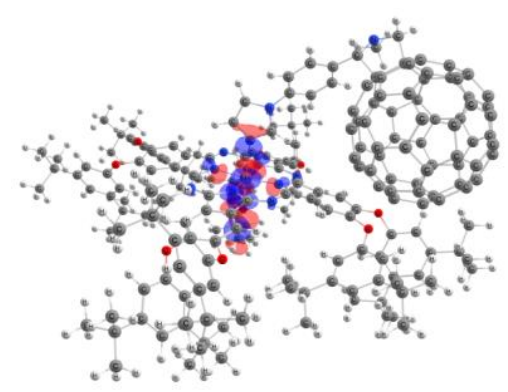

HOMO(A)

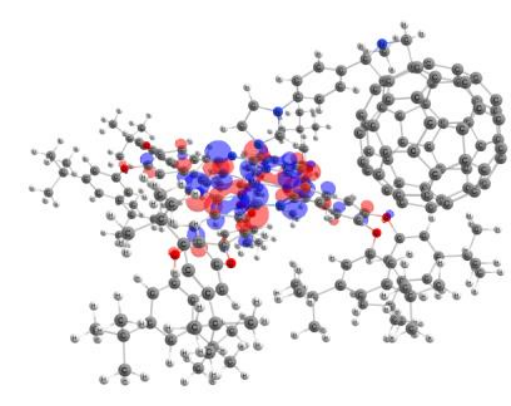

$L+1(A)$

(58\%)

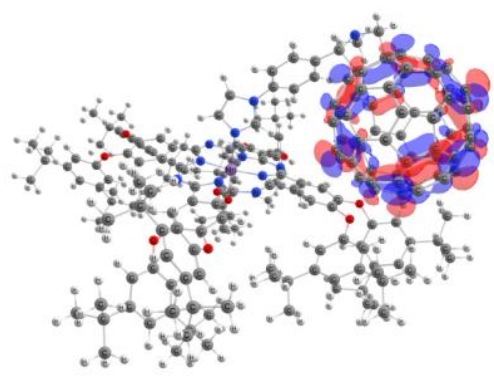

$L+2(A)$

(18\%)

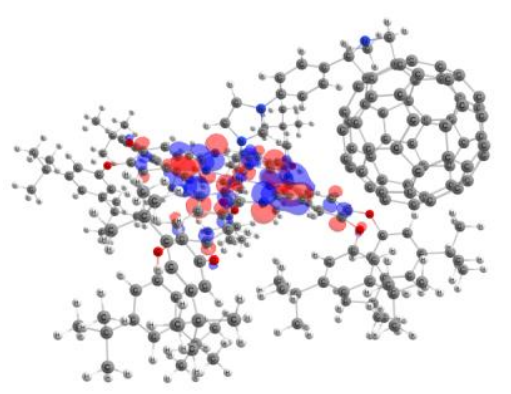

(62\%)

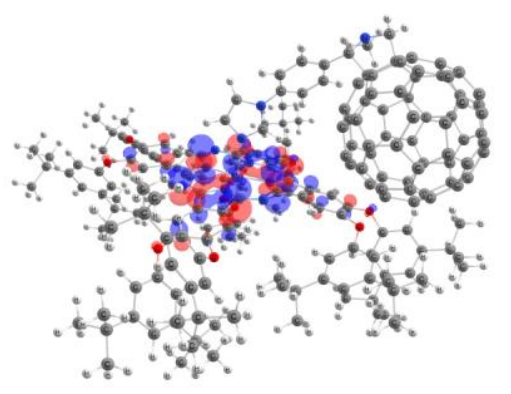

$L+2(A)$

(17\%) 

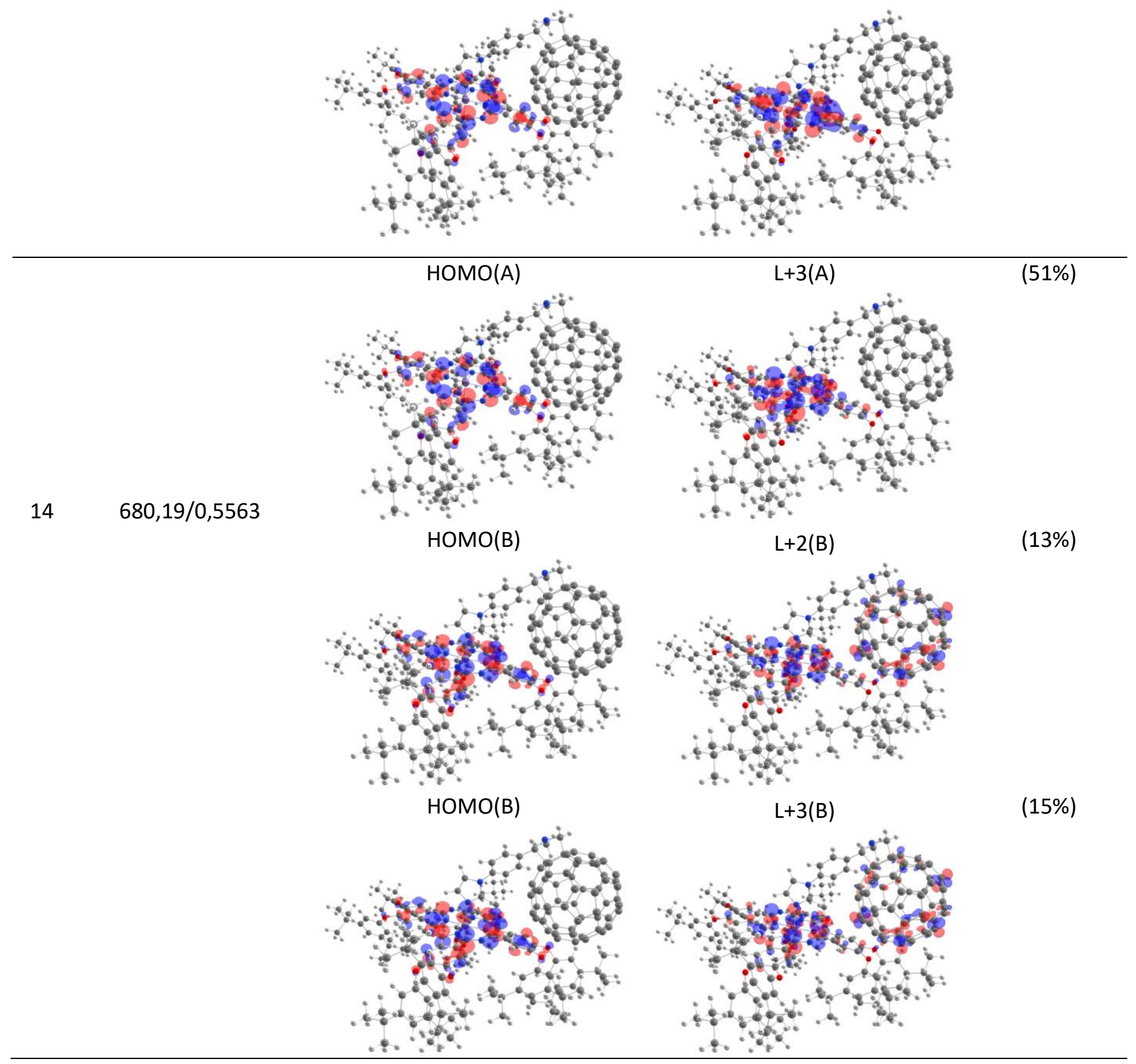
Table S8. Calculated 80 electron transitions, $\Lambda$ parameters values and $\Delta r$-index for 1:ImC60 and 2:ImC $\mathrm{C}_{60}$ by using B3LYP/6-31G level of theory.

\begin{tabular}{|c|c|c|c|c|c|c|}
\hline \multirow{2}{*}{ Excited state } & \multicolumn{3}{|c|}{$1: \operatorname{ImC}_{60}$} & \multicolumn{3}{|c|}{$2: \operatorname{ImC}_{60}$} \\
\hline & $\Lambda$ & $\Delta$ r-index, $\AA$ & Osc. Strength & $\Lambda$ & $\Delta$ r-index, $\AA$ & Osc. Strength \\
\hline 1 & 0.423601 & 3.440756 & 0.0001 & 0.611312 & 2.873016 & 0.0049 \\
\hline 2 & 0.432050 & 3.179476 & 0.0002 & 0.425440 & 1.378928 & 0.0001 \\
\hline 3 & 0.413994 & 3.421624 & 0.0001 & 0.608614 & 3.189928 & 0.0077 \\
\hline 4 & 0.429697 & 2.536098 & 0.0001 & 0.545417 & 4.836823 & 0.0 \\
\hline 5 & 0.695913 & 0.646280 & 0.0011 & 0.484301 & 6.327672 & 0.0005 \\
\hline 6 & 0.717415 & 0.332304 & 0.0017 & 0.125692 & 10.067238 & 0.0022 \\
\hline 7 & 0.067035 & 10.785326 & 0.0019 & 0.530125 & 3.530786 & 0.0004 \\
\hline 8 & 0.073503 & 10.702352 & 0.0026 & 0.534271 & 5.315573 & 0.0201 \\
\hline 9 & 0.558129 & 4.269625 & 0.0 & 0.329696 & 1.970767 & 0.0592 \\
\hline 10 & 0.414108 & 3.004227 & 0.0 & 0.503827 & 4.860840 & 0.0 \\
\hline 11 & 0.073080 & 10.750999 & 0.0026 & 0.334155 & 5.442661 & 0.1697 \\
\hline 12 & 0.064855 & 10.842127 & 0.0033 & 0.281086 & 7.275746 & 0.0987 \\
\hline 13 & 0.614385 & 2.138225 & 0.0002 & 0.396000 & 1.515540 & 0.1874 \\
\hline 14 & 0.568460 & 1.469992 & 0.0004 & 0.589937 & 2.208020 & 0.5563 \\
\hline 15 & 0.513107 & 4.279847 & 0.0 & 0.497782 & 4.744169 & 0.0 \\
\hline 16 & 0.487034 & 1.536491 & 0.0002 & 0.429522 & 5.757063 & 0.0001 \\
\hline 17 & 0.492079 & 0.959568 & 0.0014 & 0.168097 & 9.672934 & 0.0004 \\
\hline 18 & 0.506975 & 1.556535 & 0.0004 & 0.240447 & 6.130345 & 0.0096 \\
\hline 19 & 0.163401 & 10.484200 & 0.0065 & 0.444382 & 6.224701 & 0.0053 \\
\hline 20 & 0.118987 & 10.849462 & 0.0113 & 0.107527 & 10.377900 & 0.0002 \\
\hline 21 & 0.514582 & 4.469436 & 0.0 & 0.255192 & 1.758523 & 0.0077 \\
\hline 22 & 0.532801 & 4.107724 & 0.0 & 0.554770 & 4.137307 & 0.0 \\
\hline 23 & 0.681654 & 0.808965 & 0.5261 & 0.249622 & 6.834399 & 0.011 \\
\hline 24 & 0.291893 & 7.899008 & 0.0167 & 0.533492 & 3.393501 & 0.0 \\
\hline 25 & 0.336402 & 6.962517 & 0.1281 & 0.525386 & 6.390980 & 0.0001 \\
\hline 26 & 0.628669 & 1.751101 & 0.6465 & 0.033111 & 11.782887 & 0.0 \\
\hline 27 & 0.398826 & 3.691814 & 0.0291 & 0.472749 & 4.575408 & 0.0003 \\
\hline 28 & 0.594074 & 3.324673 & 0.0006 & 0.553023 & 3.592451 & 0.0 \\
\hline 29 & 0.072750 & 10.500084 & 0.0001 & 0.479562 & 4.416913 & 0.0 \\
\hline 30 & 0.352209 & 6.668923 & 0.0002 & 0.487986 & 4.091217 & 0.0 \\
\hline 31 & 0.512984 & 5.497755 & 0.0002 & 0.268581 & 8.818596 & 0.0146 \\
\hline 32 & 0.366424 & 6.713171 & 0.0004 & 0.572808 & 3.118067 & 0.0001 \\
\hline 33 & 0.462893 & 4.910850 & 0.0001 & 0.050800 & 11.562241 & 0.0 \\
\hline 34 & 0.093622 & 10.292259 & 0.0001 & 0.333584 & 5.153625 & 0.0037 \\
\hline 35 & 0.599511 & 2.712040 & 0.0 & 0.255888 & 3.864731 & 0.0049 \\
\hline 36 & 0.374733 & 7.323502 & 0.0002 & 0.495703 & 4.867000 & 0.0 \\
\hline 37 & 0.469709 & 4.856530 & 0.0 & 0.460970 & 4.446245 & 0.0005 \\
\hline 38 & 0.581256 & 3.723860 & 0.0 & 0.302010 & 5.822003 & 0.0081 \\
\hline 39 & 0.450560 & 5.530781 & 0.0004 & 0.474145 & 4.211148 & 0.0007 \\
\hline 40 & 0.370657 & 4.638674 & 0.0447 & 0.557539 & 3.523822 & 0.001 \\
\hline 41 & 0.383198 & 3.353840 & 0.0119 & 0.303754 & 6.499661 & 0.0012 \\
\hline 42 & 0.401137 & 6.082806 & 0.0009 & 0.246453 & 6.397072 & 0.0101 \\
\hline
\end{tabular}




\begin{tabular}{|c|c|c|c|c|c|c|}
\hline 43 & 0.374077 & 5.105417 & 0.0094 & 0.493099 & 4.514442 & 0.0097 \\
\hline 44 & 0.379138 & 5.835334 & 0.0061 & 0.305635 & 6.588663 & 0.0118 \\
\hline 45 & 0.537472 & 3.843212 & 0.0008 & 0.303363 & 6.515481 & 0.0411 \\
\hline 46 & 0.441159 & 4.815490 & 0.0008 & 0.416998 & 5.578786 & 0.0038 \\
\hline 47 & 0.418723 & 4.340141 & 0.0017 & 0.531753 & 3.433874 & 0.0019 \\
\hline 48 & 0.409391 & 5.244554 & 0.0037 & 0.407624 & 5.460618 & 0.0108 \\
\hline 49 & 0.414311 & 6.043075 & 0.0008 & 0.332797 & 5.721405 & 0.0079 \\
\hline 50 & 0.450839 & 5.818194 & 0.0009 & 0.377305 & 4.972296 & 0.019 \\
\hline 51 & 0.450661 & 4.951915 & 0.0004 & 0.385530 & 6.258843 & 0.0059 \\
\hline 52 & 0.574863 & 3.706597 & 0.0005 & 0.387707 & 5.515960 & 0.0109 \\
\hline 53 & 0.485133 & 5.707125 & 0.0009 & 0.327429 & 6.749325 & 0.0009 \\
\hline 54 & 0.345717 & 8.365611 & 0.0002 & 0.490247 & 4.928001 & 0.0005 \\
\hline 55 & 0.211399 & 9.453731 & 0.0004 & 0.498662 & 4.421642 & 0.001 \\
\hline 56 & 0.384332 & 6.724465 & 0.0006 & 0.302752 & 4.553277 & 0.0011 \\
\hline 57 & 0.328890 & 7.709677 & 0.0011 & 0.221427 & 1.458790 & 0.0009 \\
\hline 58 & 0.415761 & 6.276269 & 0.0002 & 0.294067 & 6.167393 & 0.0191 \\
\hline 59 & 0.393461 & 5.898106 & 0.0002 & 0.553352 & 2.768144 & 0.0001 \\
\hline 60 & 0.557398 & 2.839834 & 0.0 & 0.494229 & 4.131852 & 0.0061 \\
\hline 61 & 0.413649 & 5.942976 & 0.0011 & 0.346498 & 5.262126 & 0.0033 \\
\hline 62 & 0.182954 & 10.955276 & 0.0002 & 0.323135 & 5.429909 & 0.0018 \\
\hline 63 & 0.155817 & 9.869105 & 0.0003 & 0.391850 & 5.608823 & 0.0057 \\
\hline 64 & 0.428187 & 6.007248 & 0.0004 & 0.393099 & 5.430016 & 0.0066 \\
\hline 65 & 0.382043 & 4.040203 & 0.0011 & 0.343650 & 6.412431 & 0.0006 \\
\hline 66 & 0.340849 & 9.352395 & 0.0002 & 0.367700 & 6.286923 & 0.0103 \\
\hline 67 & 0.234146 & 8.801852 & 0.0001 & 0.352934 & 6.453399 & 0.0195 \\
\hline 68 & 0.273107 & 9.120952 & 0.0002 & 0.354954 & 5.999993 & 0.017 \\
\hline 69 & 0.328380 & 6.514008 & 0.0002 & 0.326975 & 6.136317 & 0.0662 \\
\hline 70 & 0.413398 & 5.289269 & 0.0026 & 0.475575 & 5.121728 & 0.0039 \\
\hline 71 & 0.515793 & 3.350796 & 0.0008 & 0.466506 & 4.665821 & 0.0047 \\
\hline 72 & 0.365599 & 4.116768 & 0.0008 & 0.357446 & 6.149265 & 0.008 \\
\hline 73 & 0.268861 & 6.749738 & 0.0001 & 0.128707 & 10.369206 & 0.0001 \\
\hline 74 & 0.248464 & 8.494994 & 0.0004 & 0.257945 & 7.874416 & 0.0003 \\
\hline 75 & 0.331513 & 4.346637 & 0.0003 & 0.226311 & 8.442439 & 0.001 \\
\hline 76 & 0.342839 & 7.398052 & 0.0017 & 0.506383 & 5.209111 & 0.0016 \\
\hline 77 & 0.547345 & 4.546783 & 0.0014 & 0.364661 & 6.508795 & 0.002 \\
\hline 78 & 0.199766 & 12.513465 & 0.0004 & 0.092262 & 11.960001 & 0.0006 \\
\hline 79 & 0.342906 & 7.301918 & 0.0001 & 0.344777 & 10.077390 & 0.0061 \\
\hline 80 & 0.174523 & 12.473580 & 0.0001 & 0.267914 & 5.201382 & 0.001 \\
\hline
\end{tabular}


Figure S13. Spin density distribution doublet states in the compounds 1 (a), 1:ImC60 (c) and quintet states of 2 (b) and 2:ImC60 (d) calculated by UB3LYP*+D3BJ/6-31G method (Cutoff at 0.01 a.u.).

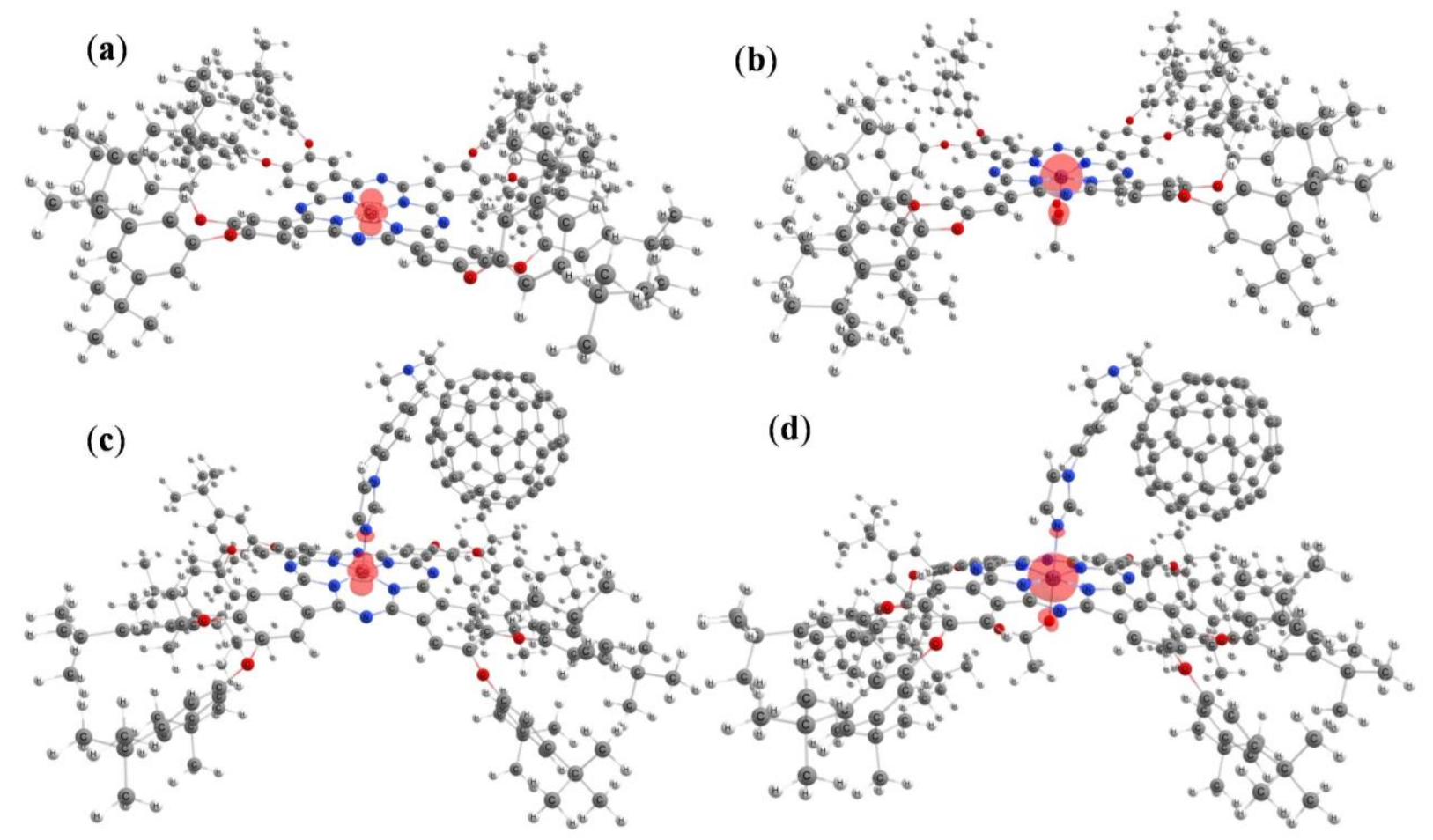

\section{Cartesian coordinates in Å for B3LYP*+D3BJ optimized geometries}

1 LS state, doublet, $\langle\mathrm{S} 2\rangle=0.756587$

Co

$\mathrm{C}$

$\mathrm{C}$

$\mathrm{C}$

$\mathrm{N}$

$\mathrm{N}$

C

C

$\mathrm{C}$

C

C

C

$\mathrm{N}$

C

C

C

C

$\mathrm{N}$

C

$\mathrm{N}$

C
$-0.08647$

$-3.97448$

$-3.21573$

$-1.80327$

$-0.7984$

0.649814

1.647517

3.06863

3.813117

2.842181

1.595154

0.46849

1.550947

$-2.92813$

$-1.75514$

$-0.62862$

2.748186

$-1.12248$

2.327276

3.209178

$-3.0152$
0.149933

$-1.46912$

$-2.64392$

$-2.26964$

$-3.14487$

3.474656

2.591228

2.971475

1.776524

0.691412

$-3.73096$

$-2.7822$

1.206866

3.272543

4.049508

3.100518

$-2.98106$

1.797545

$-1.58388$

$-0.58771$

$-0.39555$

\begin{tabular}{r|l}
2 \\
-1.8548 & $\mathrm{C}$ \\
-1.47803 & $\mathrm{C}$ \\
-1.2402 & $\mathrm{C}$ \\
-1.41858 & $\mathrm{~N}$ \\
-1.32843 & $\mathrm{~N}$ \\
-1.66986 & $\mathrm{C}$ \\
-1.74276 & $\mathrm{C}$ \\
-1.65145 & $\mathrm{C}$ \\
-1.83168 & $\mathrm{C}$ \\
-1.9835 & $\mathrm{C}$ \\
-1.52388 & $\mathrm{C}$ \\
-1.54125 & $\mathrm{~N}$ \\
-1.90919 & $\mathrm{C}$ \\
-1.91774 & $\mathrm{C}$ \\
-1.74209 & $\mathrm{C}$ \\
-1.7668 & $\mathrm{C}$ \\
-1.86651 & $\mathrm{~N}$ \\
-1.88547 & $\mathrm{C}$ \\
-1.98424 & $\mathrm{~N}$ \\
-2.0838 & $\mathrm{C}$ \\
-1.74933 & $\mathrm{C}$
\end{tabular}

2 quintet state $\langle$ S2 $\rangle=6.033521$

$\begin{array}{rrr}-3.68571 & -1.81068 & -0.21962 \\ -2.75909 & -2.89029 & -0.18986 \\ -1.43646 & -2.31181 & -0.47563 \\ -0.30512 & -3.02172 & -0.4929 \\ 0.0966 & 3.754559 & -1.35529 \\ 1.197574 & 3.021488 & -1.51569 \\ 2.539819 & 3.597844 & -1.70281 \\ 3.421401 & 2.504332 & -1.9037 \\ 2.634945 & 1.282479 & -1.74932 \\ 2.153427 & -3.21776 & -0.71231 \\ 0.882045 & -2.47885 & -0.77369 \\ 1.304611 & 1.625902 & -1.52266 \\ -3.39094 & 3.049104 & -0.84289 \\ -2.3482 & 3.982219 & -1.07065 \\ -1.10669 & 3.205008 & -1.17971 \\ 3.168504 & -2.30627 & -1.113 \\ -1.40991 & 1.846774 & -1.02882 \\ 2.516579 & -1.02793 & -1.37953 \\ 3.19748 & 0.07468 & -1.70643 \\ -2.92644 & -0.60185 & -0.5254 \\ -3.18528 & -4.2003 & 0.078783\end{array}$




\begin{tabular}{|c|c|c|c|c|c|c|c|}
\hline $\mathrm{C}$ & -3.84743 & -3.83664 & -0.84551 & $\mathrm{C}$ & 3.039804 & 4.912435 & -1.62633 \\
\hline $\mathrm{C}$ & 3.709863 & 4.188549 & -1.37089 & $\mathrm{C}$ & 2.479382 & -4.51925 & -0.29286 \\
\hline $\mathrm{C}$ & 1.702968 & -5.09236 & -1.17699 & $\mathrm{C}$ & 4.524221 & -2.67398 & -1.09593 \\
\hline $\mathrm{C}$ & 4.022781 & -3.5644 & -1.93408 & $\mathrm{C}$ & -5.05013 & -2.02145 & 0.033976 \\
\hline $\mathrm{C}$ & -5.37322 & -1.46487 & -1.36518 & $\mathrm{C}$ & 4.800219 & 2.682193 & -2.08819 \\
\hline $\mathrm{C}$ & 5.212762 & 1.773269 & -1.72382 & $\mathrm{C}$ & 5.311202 & 3.983018 & -1.97121 \\
\hline $\mathrm{C}$ & 5.860238 & 2.977037 & -1.4093 & $\mathrm{C}$ & 4.431428 & 5.09652 & -1.73166 \\
\hline $\mathrm{C}$ & 5.115756 & 4.192455 & -1.22715 & $\mathrm{C}$ & -2.63571 & 5.362316 & -1.14169 \\
\hline $\mathrm{C}$ & -1.84476 & 5.435735 & -1.50457 & $\mathrm{C}$ & -3.97282 & 5.760466 & -1.0021 \\
\hline $\mathrm{C}$ & -3.12773 & 6.007893 & -1.41363 & $\mathrm{C}$ & -5.02716 & 4.803674 & -0.79485 \\
\hline $\mathrm{C}$ & -4.30912 & 5.212029 & -1.60505 & $\mathrm{C}$ & -4.73174 & 3.434103 & -0.68852 \\
\hline $\mathrm{C}$ & -4.20922 & 3.843792 & -1.89646 & $\mathrm{C}$ & -5.48733 & -3.33013 & 0.286781 \\
\hline $\mathrm{C}$ & -6.01664 & -2.65419 & -0.98358 & $\mathrm{C}$ & -4.56219 & -4.43131 & 0.300052 \\
\hline $\mathrm{C}$ & -5.24714 & -3.83619 & -0.69171 & $\mathrm{C}$ & 4.859657 & -3.95924 & -0.65292 \\
\hline $\mathrm{C}$ & 4.144019 & -4.91047 & -1.55665 & $\mathrm{C}$ & 3.840389 & -4.89104 & -0.24568 \\
\hline $\mathrm{C}$ & 2.986365 & -5.66847 & -1.16605 & $\mathrm{~N}$ & 1.149166 & -1.1592 & -1.15398 \\
\hline $\mathrm{N}$ & 0.949445 & -1.49668 & -1.80884 & $\mathrm{~N}$ & -3.48637 & 0.609356 & -0.60115 \\
\hline $\mathrm{N}$ & -3.39244 & 0.87137 & -1.92256 & $\mathrm{~N}$ & -1.58404 & -0.93778 & -0.68498 \\
\hline $\mathrm{N}$ & -1.7198 & -0.90365 & -1.70055 & $\mathrm{C}$ & -2.78115 & 1.719302 & -0.81853 \\
\hline $\mathrm{C}$ & -2.50978 & 1.871636 & -1.96051 & $\mathrm{H}$ & -1.85639 & 6.1173 & -1.29993 \\
\hline $\mathrm{H}$ & -0.95944 & 6.063666 & -1.34829 & $\mathrm{H}$ & -5.50963 & 2.685717 & -0.5118 \\
\hline $\mathrm{H}$ & -5.10296 & 3.232079 & -2.05884 & $\mathrm{H}$ & -5.77494 & -1.19788 & 0.030761 \\
\hline $\mathrm{H}$ & -5.97142 & -0.56551 & -1.5536 & $\mathrm{H}$ & -2.49009 & -5.04805 & 0.099263 \\
\hline $\mathrm{H}$ & -3.28457 & -4.75089 & -0.6216 & $\mathrm{H}$ & 1.716676 & -5.23526 & 0.036008 \\
\hline $\mathrm{H}$ & 0.83556 & -5.69353 & -0.87919 & $\mathrm{H}$ & 5.3199 & -1.97665 & -1.38439 \\
\hline $\mathrm{H}$ & 4.894487 & -2.98001 & -2.24617 & $\mathrm{H}$ & 5.456928 & 1.828743 & -2.28559 \\
\hline $\mathrm{H}$ & 5.805406 & 0.857486 & -1.83705 & $\mathrm{H}$ & 2.390177 & 5.777883 & -1.44976 \\
\hline $\mathrm{H}$ & 3.152479 & 5.119421 & -1.2114 & $\mathrm{C}$ & 5.244617 & -6.88114 & -0.00334 \\
\hline $\mathrm{C}$ & 4.056533 & -7.39535 & 0.128554 & $\mathrm{C}$ & 5.669458 & -7.04819 & -1.33232 \\
\hline $\mathrm{C}$ & 4.584546 & -8.68793 & -0.01524 & $\mathrm{C}$ & 5.90578 & -7.48081 & 1.071093 \\
\hline $\mathrm{C}$ & 4.435573 & -6.55142 & 1.185489 & $\mathrm{C}$ & 6.833164 & -7.79663 & -1.59082 \\
\hline $\mathrm{C}$ & 5.536575 & -9.15985 & 0.913492 & $\mathrm{H}$ & 5.111978 & -6.55353 & -2.1343 \\
\hline $\mathrm{H}$ & 4.24458 & -9.28743 & -0.86607 & $\mathrm{C}$ & 7.063468 & -8.26049 & 0.836392 \\
\hline $\mathrm{C}$ & 5.404525 & -6.9925 & 2.113219 & $\mathrm{H}$ & 5.517379 & -7.30389 & 2.079964 \\
\hline $\mathrm{H}$ & 3.991977 & -5.55316 & 1.256809 & $\mathrm{C}$ & 7.510933 & -8.39368 & -0.49443 \\
\hline $\mathrm{C}$ & 5.934263 & -8.29422 & 1.960303 & $\mathrm{H}$ & 8.417924 & -8.97304 & -0.6961 \\
\hline $\mathrm{H}$ & 6.687167 & -8.64312 & 2.67791 & $\mathrm{C}$ & 6.943687 & -4.24182 & 0.532356 \\
\hline $\mathrm{C}$ & 6.517009 & -5.04629 & -1.01946 & $\mathrm{C}$ & 8.300516 & -4.58797 & 0.415664 \\
\hline $\mathrm{C}$ & 7.665678 & -5.83883 & -1.19135 & $\mathrm{C}$ & 6.380585 & -3.85062 & 1.757727 \\
\hline $\mathrm{C}$ & 6.569498 & -3.82494 & -0.32424 & $\mathrm{C}$ & 9.143524 & -4.50719 & 1.542049 \\
\hline $\mathrm{C}$ & 8.905993 & -5.39981 & -0.68553 & $\mathrm{H}$ & 8.661716 & -4.90232 & -0.5681 \\
\hline $\mathrm{H}$ & 7.550433 & -6.79183 & -1.71734 & $\mathrm{C}$ & 7.201477 & -3.7872 & 2.90759 \\
\hline $\mathrm{C}$ & 7.810209 & -3.34187 & 0.150985 & $\mathrm{H}$ & 5.317849 & -3.59746 & 1.804205 \\
\hline $\mathrm{H}$ & 5.660537 & -3.24396 & -0.1643 & $\mathrm{C}$ & 8.573502 & -4.10165 & 2.774935 \\
\hline $\mathrm{C}$ & 8.958906 & -4.14272 & -0.03349 & $\mathrm{H}$ & 9.217364 & -4.03755 & 3.660153 \\
\hline $\mathrm{H}$ & 9.923997 & -3.77856 & 0.338928 & $\mathrm{C}$ & -6.1741 & -6.15107 & 0.962261 \\
\hline $\mathrm{C}$ & -6.75463 & -5.10143 & 0.732667 & $\mathrm{C}$ & -6.523 & -5.8836 & 2.297572 \\
\hline $\mathrm{C}$ & -6.86071 & -4.0913 & 1.705658 & $\mathrm{C}$ & -7.01346 & -6.85277 & 0.097199 \\
\hline
\end{tabular}




\begin{tabular}{|c|c|c|c|c|c|c|c|}
\hline $\mathrm{C}$ & -7.56124 & -6.24426 & 0.765285 & $\mathrm{C}$ & -7.78609 & -6.28394 & 2.768897 \\
\hline $\mathrm{C}$ & -7.84447 & -4.19882 & 2.707331 & $\mathrm{H}$ & -5.81545 & -5.3316 & 2.925201 \\
\hline $\mathrm{H}$ & -6.1975 & -3.22383 & 1.650656 & $\mathrm{C}$ & -8.28672 & -7.28138 & 0.55022 \\
\hline $\mathrm{C}$ & -8.52628 & -6.39683 & 1.787655 & $\mathrm{H}$ & -6.67117 & -7.03323 & -0.92847 \\
\hline $\mathrm{H}$ & -7.42651 & -6.99243 & -0.02443 & $\mathrm{C}$ & -8.65003 & -6.97725 & 1.876956 \\
\hline $\mathrm{C}$ & -8.66305 & -5.35744 & 2.733313 & $\mathrm{H}$ & -9.63615 & -7.28325 & 2.242035 \\
\hline $\mathrm{H}$ & -9.42681 & -5.44142 & 3.513542 & $\mathrm{C}$ & -7.78385 & -3.53546 & -0.43636 \\
\hline $\mathrm{C}$ & -8.28991 & -3.48074 & -1.33263 & $\mathrm{C}$ & -9.12248 & -3.57877 & -0.00804 \\
\hline $\mathrm{C}$ & -9.58413 & -3.43612 & -0.80074 & $\mathrm{C}$ & -7.44278 & -3.51698 & -1.79889 \\
\hline $\mathrm{C}$ & -7.94392 & -4.36583 & -2.36895 & $\mathrm{C}$ & -10.1626 & -3.56909 & -0.95818 \\
\hline $\mathrm{C}$ & -10.5836 & -4.29967 & -1.30384 & $\mathrm{H}$ & -9.30957 & -3.62626 & 1.06892 \\
\hline $\mathrm{H}$ & -9.7878 & -2.71893 & 0.002718 & $\mathrm{C}$ & -8.4705 & -3.50896 & -2.77003 \\
\hline $\mathrm{C}$ & -8.91353 & -5.26207 & -2.86151 & $\mathrm{H}$ & -6.38856 & -3.50178 & -2.08942 \\
\hline $\mathrm{H}$ & -6.92548 & -4.35545 & -2.76653 & $\mathrm{C}$ & -9.81393 & -3.52029 & -2.33085 \\
\hline $\mathrm{C}$ & -10.2236 & -5.21217 & -2.31926 & $\mathrm{H}$ & -10.6141 & -3.50121 & -3.08081 \\
\hline $\mathrm{H}$ & -10.9777 & -5.90483 & -2.70676 & $\mathrm{C}$ & -7.32665 & 4.438536 & -0.29749 \\
\hline $\mathrm{C}$ & -6.56703 & 5.333516 & -0.72445 & $\mathrm{C}$ & -7.74961 & 4.497206 & 1.037025 \\
\hline $\mathrm{C}$ & -6.43029 & 4.201924 & 0.099671 & $\mathrm{C}$ & -7.86289 & 3.527935 & -1.22056 \\
\hline $\mathrm{C}$ & -7.77981 & 6.041041 & -0.79645 & $\mathrm{C}$ & -8.745 & 3.598169 & 1.485759 \\
\hline $\mathrm{C}$ & -7.54616 & 3.716355 & 0.817868 & $\mathrm{H}$ & -7.28503 & 5.242529 & 1.691791 \\
\hline $\mathrm{H}$ & -5.46987 & 3.690501 & 0.170659 & $\mathrm{C}$ & -8.83232 & 2.594657 & -0.78863 \\
\hline $\mathrm{C}$ & -8.89568 & 5.600761 & -0.05509 & $\mathrm{H}$ & -7.48451 & 3.539546 & -2.2483 \\
\hline $\mathrm{H}$ & -7.8122 & 6.930547 & -1.4336 & $\mathrm{C}$ & -9.25378 & 2.656129 & 0.561128 \\
\hline $\mathrm{C}$ & -8.76196 & 4.429854 & 0.732006 & $\mathrm{H}$ & -10.0029 & 1.933402 & 0.907372 \\
\hline $\mathrm{H}$ & -9.63079 & 4.066567 & 1.294258 & $\mathrm{C}$ & -4.80175 & 7.750131 & 0.040818 \\
\hline $\mathrm{C}$ & -4.07332 & 7.852626 & -0.18485 & $\mathrm{C}$ & -4.57217 & 7.261384 & 1.337554 \\
\hline $\mathrm{C}$ & -4.2821 & 7.139372 & 1.006618 & $\mathrm{C}$ & -5.51725 & 8.937846 & -0.1861 \\
\hline $\mathrm{C}$ & -4.67026 & 9.10123 & -0.41805 & $\mathrm{C}$ & -5.06983 & 7.97653 & 2.451721 \\
\hline $\mathrm{C}$ & -5.14488 & 7.669763 & 1.99086 & $\mathrm{H}$ & -4.0056 & 6.334084 & 1.46543 \\
\hline $\mathrm{H}$ & -3.78904 & 6.171315 & 1.142453 & $\mathrm{C}$ & -6.01952 & 9.669928 & 0.908884 \\
\hline $\mathrm{C}$ & -5.52296 & 9.659304 & 0.557952 & $\mathrm{H}$ & -5.66821 & 9.252945 & -1.22357 \\
\hline $\mathrm{H}$ & -4.46428 & 9.596835 & -1.37232 & $\mathrm{C}$ & -5.78277 & 9.17319 & 2.215061 \\
\hline $\mathrm{C}$ & -5.7489 & 8.924345 & 1.746184 & $\mathrm{H}$ & -6.16398 & 9.741687 & 3.071549 \\
\hline $\mathrm{H}$ & -6.4216 & 9.341915 & 2.505451 & $\mathrm{C}$ & 6.035673 & 6.6674 & -0.84992 \\
\hline $\mathrm{C}$ & 7.007901 & 5.738076 & -0.993 & $\mathrm{C}$ & 6.257589 & 5.990149 & 0.360133 \\
\hline $\mathrm{C}$ & 7.680799 & 6.243112 & 0.121903 & $\mathrm{C}$ & 6.889292 & 7.685832 & -1.30022 \\
\hline $\mathrm{C}$ & 7.622243 & 5.628761 & -2.2526 & $\mathrm{C}$ & 7.397146 & 6.305377 & 1.132015 \\
\hline $\mathrm{C}$ & 9.036697 & 6.634133 & 0.008357 & $\mathrm{H}$ & 5.556123 & 5.209153 & 0.672014 \\
\hline $\mathrm{H}$ & 7.135398 & 6.299095 & 1.070506 & $\mathrm{C}$ & 8.022635 & 8.03391 & -0.53482 \\
\hline $\mathrm{C}$ & 8.976667 & 5.986712 & -2.38729 & $\mathrm{H}$ & 6.651756 & 8.167395 & -2.25441 \\
\hline $\mathrm{H}$ & 7.045344 & 5.226047 & -3.09143 & $\mathrm{C}$ & 8.258915 & 7.325308 & 0.667104 \\
\hline $\mathrm{C}$ & 9.661273 & 6.487105 & -1.24697 & $\mathrm{H}$ & 9.14736 & 7.574543 & 1.260359 \\
\hline $\mathrm{H}$ & 10.71552 & 6.763329 & -1.35345 & $\mathrm{C}$ & 7.644205 & 3.498631 & -1.47875 \\
\hline $\mathrm{C}$ & 7.832809 & 2.806367 & -0.03497 & $\mathrm{C}$ & 8.962615 & 3.829371 & -1.83765 \\
\hline $\mathrm{C}$ & 9.238236 & 2.786945 & -0.02034 & $\mathrm{C}$ & 7.373266 & 2.491993 & -0.53407 \\
\hline $\mathrm{C}$ & 7.078749 & 2.675408 & 1.142628 & $\mathrm{C}$ & 10.04326 & 3.124676 & -1.26894 \\
\hline $\mathrm{C}$ & 9.924388 & 2.590963 & 1.195296 & $\mathrm{H}$ & 9.104443 & 4.644277 & -2.55437 \\
\hline $\mathrm{H}$ & 9.757113 & 2.91986 & -0.97419 & $\mathrm{C}$ & 8.43775 & 1.741248 & 0.014967 \\
\hline
\end{tabular}




\begin{tabular}{|c|c|c|c|c|c|c|c|}
\hline $\mathrm{C}$ & 7.745549 & 2.498673 & 2.377215 & $\mathrm{H}$ & 6.344703 & 2.282215 & -0.2375 \\
\hline $\mathrm{H}$ & 5.987176 & 2.704926 & 1.087463 & $\mathrm{C}$ & 9.757688 & 2.074689 & -0.36148 \\
\hline $\mathrm{C}$ & 9.158192 & 2.444372 & 2.378931 & $\mathrm{H}$ & 10.59056 & 1.504318 & 0.067213 \\
\hline $\mathrm{H}$ & 9.678919 & 2.291961 & 3.331453 & $\mathrm{O}$ & -4.29558 & 7.111021 & -1.10611 \\
\hline $\mathrm{O}$ & -3.21624 & 7.377288 & -1.19856 & $\mathrm{O}$ & -6.30358 & 5.329186 & -0.70621 \\
\hline $\mathrm{O}$ & -5.53145 & 5.864751 & -1.51771 & $\mathrm{O}$ & -6.83442 & -3.53534 & 0.593318 \\
\hline $\mathrm{O}$ & -7.38785 & -2.55667 & -0.77896 & $\mathrm{O}$ & -4.90867 & -5.75985 & 0.460994 \\
\hline $\mathrm{O}$ & -5.82652 & -5.0457 & -0.32426 & $\mathrm{O}$ & 4.097598 & -6.12534 & 0.323595 \\
\hline $\mathrm{O}$ & 3.095862 & -7.01169 & -0.82988 & $\mathrm{O}$ & 6.211896 & -4.30764 & -0.66112 \\
\hline $\mathrm{O}$ & 5.349141 & -5.595 & -1.58056 & $\mathrm{O}$ & 6.657709 & 4.273816 & -2.11889 \\
\hline $\mathrm{O}$ & 7.253954 & 2.951715 & -1.30291 & $\mathrm{O}$ & 4.918472 & 6.393565 & -1.66689 \\
\hline $\mathrm{O}$ & 5.654607 & 5.390046 & -0.791 & $\mathrm{C}$ & -8.26982 & -5.96971 & 4.211123 \\
\hline $\mathrm{C}$ & -8.06934 & -3.06934 & 3.750818 & $\mathrm{C}$ & -8.62515 & -7.30163 & 4.946432 \\
\hline $\mathrm{C}$ & -7.9312 & -3.64508 & 5.195792 & $\mathrm{C}$ & -7.18859 & -5.22426 & 5.044746 \\
\hline $\mathrm{C}$ & -7.04791 & -1.90646 & 3.587299 & $\mathrm{C}$ & -9.53901 & -5.06053 & 4.135284 \\
\hline $\mathrm{C}$ & -9.50497 & -2.48318 & 3.555127 & $\mathrm{H}$ & -9.43118 & -7.85459 & 4.426452 \\
\hline $\mathrm{H}$ & -8.67096 & -4.44521 & 5.391026 & $\mathrm{H}$ & -7.73908 & -7.96489 & 5.003375 \\
\hline $\mathrm{H}$ & -6.91999 & -4.0712 & 5.351546 & $\mathrm{H}$ & -8.96907 & -7.09018 & 5.979807 \\
\hline $\mathrm{H}$ & -8.09075 & -2.84347 & 5.9461 & $\mathrm{H}$ & -6.9228 & -4.24921 & 4.589828 \\
\hline $\mathrm{H}$ & -7.12153 & -1.43838 & 2.586084 & $\mathrm{H}$ & -7.57634 & -5.02632 & 6.063851 \\
\hline $\mathrm{H}$ & -7.2532 & -1.12175 & 4.342777 & $\mathrm{H}$ & -6.26347 & -5.82618 & 5.146818 \\
\hline $\mathrm{H}$ & -6.00523 & -2.25265 & 3.734571 & $\mathrm{H}$ & -9.89985 & -4.81739 & 5.15592 \\
\hline $\mathrm{H}$ & -9.69752 & -1.67613 & 4.291876 & $\mathrm{H}$ & -9.30674 & -4.11056 & 3.613684 \\
\hline $\mathrm{H}$ & -9.61322 & -2.05655 & 2.537827 & $\mathrm{H}$ & -10.3656 & -5.55566 & 3.59036 \\
\hline $\mathrm{H}$ & -10.2834 & -3.25958 & 3.683154 & $\mathrm{C}$ & -9.21243 & -8.06015 & -0.42196 \\
\hline $\mathrm{C}$ & -9.37072 & -7.69954 & 1.843392 & $\mathrm{C}$ & -10.6418 & -8.25811 & 0.159905 \\
\hline $\mathrm{C}$ & -10.4667 & -7.64956 & 2.946714 & $\mathrm{C}$ & -9.33577 & -7.28099 & -1.7707 \\
\hline $\mathrm{C}$ & -10.0667 & -7.94349 & 0.466722 & $\mathrm{C}$ & -8.58599 & -9.46666 & -0.68992 \\
\hline $\mathrm{C}$ & -8.41641 & -8.89778 & 2.156525 & $\mathrm{H}$ & -10.6312 & -8.86983 & 1.083875 \\
\hline $\mathrm{H}$ & -10.0262 & -7.54427 & 3.958094 & $\mathrm{H}$ & -11.1256 & -7.288 & 0.390153 \\
\hline $\mathrm{H}$ & -11.175 & -6.81251 & 2.787074 & $\mathrm{H}$ & -11.2746 & -8.78521 & -0.58155 \\
\hline $\mathrm{H}$ & -11.0492 & -8.59227 & 2.932202 & $\mathrm{H}$ & -8.36425 & -7.21329 & -2.29618 \\
\hline $\mathrm{H}$ & -9.3311 & -8.084 & -0.34673 & $\mathrm{H}$ & -10.0459 & -7.79915 & -2.44737 \\
\hline $\mathrm{H}$ & -10.6983 & -8.85436 & 0.514326 & $\mathrm{H}$ & -9.69569 & -6.24917 & -1.60435 \\
\hline $\mathrm{H}$ & -10.7056 & -7.08465 & 0.187935 & $\mathrm{H}$ & -9.21813 & -10.0428 & -1.39688 \\
\hline $\mathrm{H}$ & -8.98974 & -9.84689 & 2.201951 & $\mathrm{H}$ & -7.57261 & -9.3771 & -1.12805 \\
\hline $\mathrm{H}$ & -7.63505 & -9.00476 & 1.379135 & $\mathrm{H}$ & -8.49934 & -10.0446 & 0.252021 \\
\hline $\mathrm{H}$ & -7.90852 & -8.74959 & 3.130525 & $\mathrm{C}$ & 7.77836 & -8.94711 & 2.031104 \\
\hline $\mathrm{C}$ & 5.911943 & -6.08934 & 3.270683 & $\mathrm{C}$ & 9.219625 & -9.40662 & 1.662125 \\
\hline $\mathrm{C}$ & 7.453502 & -5.88554 & 3.114218 & $\mathrm{C}$ & 7.869532 & -7.96493 & 3.242737 \\
\hline $\mathrm{C}$ & 5.234121 & -4.68883 & 3.264096 & $\mathrm{C}$ & 6.946058 & -10.2021 & 2.45028 \\
\hline $\mathrm{C}$ & 5.607208 & -6.77558 & 4.64018 & $\mathrm{H}$ & 9.215461 & -10.1961 & 0.884868 \\
\hline $\mathrm{H}$ & 7.998582 & -6.84755 & 3.173879 & $\mathrm{H}$ & 9.834009 & -8.56122 & 1.294296 \\
\hline $\mathrm{H}$ & 7.691085 & -5.41917 & 2.140119 & $\mathrm{H}$ & 9.717642 & -9.82743 & 2.558223 \\
\hline $\mathrm{H}$ & 7.837527 & -5.22754 & 3.921247 & $\mathrm{H}$ & 6.872915 & -7.75212 & 3.67446 \\
\hline $\mathrm{H}$ & 4.136407 & -4.76267 & 3.400556 & $\mathrm{H}$ & 8.487827 & -8.41088 & 4.048063 \\
\hline $\mathrm{H}$ & 5.636407 & -4.07834 & 4.097259 & $\mathrm{H}$ & 8.318017 & -6.99912 & 2.944369 \\
\hline $\mathrm{H}$ & 5.436667 & -4.14745 & 2.320318 & $\mathrm{H}$ & 7.419366 & -10.7108 & 3.31585 \\
\hline
\end{tabular}




\begin{tabular}{|c|c|c|c|c|c|c|c|}
\hline $\mathrm{H}$ & 5.969753 & -6.14192 & 5.47584 & $\mathrm{H}$ & 5.915183 & -9.91543 & 2.737842 \\
\hline $\mathrm{H}$ & 4.516981 & -6.92994 & 4.767925 & $\mathrm{H}$ & 6.878418 & -10.926 & 1.613667 \\
\hline $\mathrm{H}$ & 6.102203 & -7.76267 & 4.721225 & $\mathrm{C}$ & 7.409817 & -7.95279 & -3.0244 \\
\hline $\mathrm{C}$ & 6.161809 & -10.5784 & 0.812938 & $\mathrm{C}$ & 7.514152 & -9.46831 & -3.38814 \\
\hline $\mathrm{C}$ & 5.829193 & -11.3797 & 2.112816 & $\mathrm{C}$ & 6.52425 & -7.25256 & -4.09483 \\
\hline $\mathrm{C}$ & 5.617668 & -11.3779 & -0.40552 & $\mathrm{C}$ & 8.830715 & -7.30221 & -3.06895 \\
\hline $\mathrm{C}$ & 7.711626 & -10.4512 & 0.660718 & $\mathrm{H}$ & 8.173711 & -10.0146 & -2.68645 \\
\hline $\mathrm{H}$ & 6.233384 & -10.8805 & 3.014583 & $\mathrm{H}$ & 6.5149 & -9.94702 & -3.35812 \\
\hline $\mathrm{H}$ & 4.732672 & -11.477 & 2.2414 & $\mathrm{H}$ & 7.927888 & -9.59103 & -4.41014 \\
\hline $\mathrm{H}$ & 6.266133 & -12.3982 & 2.059758 & $\mathrm{H}$ & 6.449637 & -6.16192 & -3.91277 \\
\hline $\mathrm{H}$ & 5.847699 & -10.8704 & -1.36358 & $\mathrm{H}$ & 6.97134 & -7.39705 & -5.09848 \\
\hline $\mathrm{H}$ & 6.089315 & -12.3805 & -0.43244 & $\mathrm{H}$ & 5.499649 & -7.67476 & -4.11843 \\
\hline $\mathrm{H}$ & 4.520778 & -11.5225 & -0.34234 & $\mathrm{H}$ & 9.265039 & -7.3925 & -4.08592 \\
\hline $\mathrm{H}$ & 8.176857 & -11.4562 & 0.592364 & $\mathrm{H}$ & 8.771123 & -6.22594 & -2.81107 \\
\hline $\mathrm{H}$ & 7.966816 & -9.88771 & -0.25844 & $\mathrm{H}$ & 9.525701 & -7.78575 & -2.35561 \\
\hline $\mathrm{H}$ & 8.167052 & -9.92168 & 1.519763 & $\mathrm{C}$ & -9.27124 & 3.602931 & 2.946943 \\
\hline $\mathrm{C}$ & -7.46231 & 2.406654 & 1.653814 & $\mathrm{C}$ & -8.98991 & 2.213068 & 3.603987 \\
\hline $\mathrm{C}$ & -8.45918 & 1.361873 & 1.052224 & $\mathrm{C}$ & -8.58528 & 4.699598 & 3.80958 \\
\hline $\mathrm{C}$ & -6.03709 & 1.778902 & 1.633597 & $\mathrm{C}$ & -10.8097 & 3.876112 & 2.938212 \\
\hline $\mathrm{C}$ & -7.84869 & 2.69721 & 3.139207 & $\mathrm{H}$ & -9.4999 & 1.392543 & 3.062885 \\
\hline $\mathrm{H}$ & -9.50184 & 1.734488 & 1.073101 & $\mathrm{H}$ & -7.90341 & 1.994457 & 3.607632 \\
\hline $\mathrm{H}$ & -8.20009 & 1.132294 & -0.00052 & $\mathrm{H}$ & -9.35077 & 2.205334 & 4.652964 \\
\hline $\mathrm{H}$ & -8.42031 & 0.416764 & 1.630874 & $\mathrm{H}$ & -8.76089 & 5.712253 & 3.395415 \\
\hline $\mathrm{H}$ & -5.2813 & 2.46091 & 2.072038 & $\mathrm{H}$ & -8.99781 & 4.67922 & 4.837875 \\
\hline $\mathrm{H}$ & -6.03577 & 0.848306 & 2.234369 & $\mathrm{H}$ & -7.49158 & 4.538519 & 3.88339 \\
\hline $\mathrm{H}$ & -5.71336 & 1.514129 & 0.607223 & $\mathrm{H}$ & -11.2042 & 3.884621 & 3.975026 \\
\hline $\mathrm{H}$ & -7.80513 & 1.761732 & 3.733814 & $\mathrm{H}$ & -11.0298 & 4.858196 & 2.474118 \\
\hline $\mathrm{H}$ & -7.15225 & 3.43018 & 3.590846 & $\mathrm{H}$ & -11.3589 & 3.10085 & 2.36965 \\
\hline $\mathrm{H}$ & -8.87409 & 3.106523 & 3.222928 & $\mathrm{C}$ & -9.37832 & 1.47688 & -1.71868 \\
\hline $\mathrm{C}$ & -10.2454 & 6.369911 & -0.06327 & $\mathrm{C}$ & -10.9397 & 1.483288 & -1.70899 \\
\hline $\mathrm{C}$ & -10.5042 & 6.939802 & 1.369005 & $\mathrm{C}$ & -8.90497 & 1.653855 & -3.19034 \\
\hline $\mathrm{C}$ & -10.2413 & 7.557196 & -1.06805 & $\mathrm{C}$ & -8.85335 & 0.099714 & -1.19412 \\
\hline $\mathrm{C}$ & -11.4051 & 5.401219 & -0.46026 & $\mathrm{H}$ & -11.35 & 1.289235 & -0.699 \\
\hline $\mathrm{H}$ & -10.5402 & 6.132634 & 2.126252 & $\mathrm{H}$ & -11.3312 & 2.46093 & -2.05473 \\
\hline $\mathrm{H}$ & -9.69698 & 7.64101 & 1.659057 & $\mathrm{H}$ & -11.3266 & 0.693239 & -2.3834 \\
\hline $\mathrm{H}$ & -11.4699 & 7.485462 & 1.398659 & $\mathrm{H}$ & -7.80296 & 1.579252 & -3.28167 \\
\hline $\mathrm{H}$ & -10.0611 & 7.213166 & -2.10617 & $\mathrm{H}$ & -9.34516 & 0.854246 & -3.8175 \\
\hline $\mathrm{H}$ & -11.2254 & 8.066314 & -1.04475 & $\mathrm{H}$ & -9.22552 & 2.629253 & -3.60862 \\
\hline $\mathrm{H}$ & -9.46938 & 8.307418 & -0.8086 & $\mathrm{H}$ & -9.22414 & -0.73237 & -1.8236 \\
\hline $\mathrm{H}$ & -12.3692 & 5.949374 & -0.4824 & $\mathrm{H}$ & -7.74515 & 0.076514 & -1.21066 \\
\hline $\mathrm{H}$ & -11.2276 & 4.968723 & -1.4652 & $\mathrm{H}$ & -9.18059 & -0.0913 & -0.15391 \\
\hline $\mathrm{H}$ & -11.51 & 4.564078 & 0.256444 & $\mathrm{C}$ & -6.82954 & 10.98322 & 0.72504 \\
\hline $\mathrm{C}$ & -6.22777 & 11.02915 & 0.358376 & $\mathrm{C}$ & -6.10268 & 12.14886 & 1.470165 \\
\hline $\mathrm{C}$ & -5.82077 & 11.99553 & 1.517034 & $\mathrm{C}$ & -6.97776 & 11.38043 & -0.7717 \\
\hline $\mathrm{C}$ & -5.84324 & 11.69952 & -0.99199 & $\mathrm{C}$ & -8.25976 & 10.79131 & 1.32557 \\
\hline $\mathrm{C}$ & -7.77579 & 10.81733 & 0.375585 & $\mathrm{H}$ & -6.0143 & 11.94918 & 2.555652 \\
\hline $\mathrm{H}$ & -6.11913 & 11.59753 & 2.506069 & $\mathrm{H}$ & -5.0808 & 12.29351 & 1.06627 \\
\hline $\mathrm{H}$ & -4.7234 & 12.14988 & 1.529778 & $\mathrm{H}$ & -6.66548 & 13.09647 & 1.3423 \\
\hline
\end{tabular}




\begin{tabular}{|c|c|c|c|c|c|c|c|}
\hline $\mathrm{H}$ & -6.31046 & 12.98217 & 1.384065 & $\mathrm{H}$ & -7.51267 & 10.60175 & -1.35092 \\
\hline $\mathrm{H}$ & -6.13706 & 11.07387 & -1.85829 & $\mathrm{H}$ & -7.56088 & 12.31969 & -0.85 \\
\hline $\mathrm{H}$ & -6.36602 & 12.67222 & -1.08607 & $\mathrm{H}$ & -5.99195 & 11.55343 & -1.2475 \\
\hline $\mathrm{H}$ & -4.75394 & 11.89331 & -1.05594 & $\mathrm{H}$ & -8.85508 & 11.71992 & 1.205612 \\
\hline 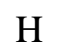 & -8.3004 & 11.7872 & 0.252346 & $\mathrm{H}$ & -8.79225 & 9.966778 & 0.8106 \\
\hline $\mathrm{H}$ & -8.08503 & 10.14774 & -0.45129 & $\mathrm{H}$ & -8.21927 & 10.54749 & 2.40485 \\
\hline $\mathrm{H}$ & -8.11611 & 10.36205 & 1.325679 & $\mathrm{C}$ & -4.78631 & 7.507226 & 3.90658 \\
\hline $\mathrm{C}$ & -5.45482 & 6.915128 & 3.312573 & $\mathrm{C}$ & -6.07517 & 7.612873 & 4.78193 \\
\hline $\mathrm{C}$ & -6.99222 & 6.647941 & 3.3959 & $\mathrm{C}$ & -4.29044 & 6.03146 & 3.95765 \\
\hline $\mathrm{C}$ & -4.71742 & 5.54772 & 3.395461 & $\mathrm{C}$ & -3.67771 & 8.428248 & 4.51226 \\
\hline$C$ & -5.00599 & 7.789379 & 4.526836 & $\mathrm{H}$ & -6.42798 & 8.657103 & 4.8794 \\
\hline $\mathrm{H}$ & -7.56756 & 7.593798 & 3.406829 & $\mathrm{H}$ & -6.89877 & 7.012838 & 4.34995 \\
\hline $\mathrm{H}$ & -7.33545 & 6.050342 & 2.531097 & $\mathrm{H}$ & -5.8695 & 7.234732 & 5.804174 \\
\hline $\mathrm{H}$ & -7.23586 & 6.092482 & 4.32518 & $\mathrm{H}$ & -3.3104 & 5.906054 & 3.45689 \\
\hline $\mathrm{H}$ & -3.61616 & 5.672495 & 3.370482 & $\mathrm{H}$ & -4.16558 & 5.716737 & 5.012854 \\
\hline $\mathrm{H}$ & -4.97669 & 5.043224 & 4.347817 & $\mathrm{H}$ & -5.01385 & 5.341924 & 3.47775 \\
\hline $\mathrm{H}$ & -5.01545 & 4.878586 & 2.565697 & $\mathrm{H}$ & -3.44235 & 8.121724 & 5.55277 \\
\hline $\mathrm{H}$ & -5.22456 & 7.26479 & 5.479903 & $\mathrm{H}$ & -2.74844 & 8.368333 & 3.911439 \\
\hline $\mathrm{H}$ & -3.91724 & 7.992826 & 4.482591 & $\mathrm{H}$ & -4.00528 & 9.486423 & 4.527422 \\
\hline $\mathrm{H}$ & -5.53296 & 8.76298 & 4.545215 & $\mathrm{C}$ & 7.73551 & 5.558824 & 2.45114 \\
\hline $\mathrm{C}$ & 9.768417 & 7.220136 & 1.245518 & $\mathrm{C}$ & 7.790029 & 6.581669 & 3.63035 \\
\hline $\mathrm{C}$ & 9.266231 & 8.683333 & 1.470257 & $\mathrm{C}$ & 6.680025 & 4.469463 & 2.798162 \\
\hline $\mathrm{C}$ & 9.448493 & 6.366559 & 2.514647 & $\mathrm{C}$ & 9.124517 & 4.859881 & 2.29425 \\
\hline $\mathrm{C}$ & 11.31292 & 7.249972 & 1.051836 & $\mathrm{H}$ & 8.558576 & 7.360793 & 3.461448 \\
\hline $\mathrm{H}$ & 9.49653 & 9.317205 & 0.590628 & $\mathrm{H}$ & 6.812884 & 7.090087 & 3.75479 \\
\hline $\mathrm{H}$ & 8.170132 & 8.707039 & 1.629446 & $\mathrm{H}$ & 8.035567 & 6.060644 & 4.578769 \\
\hline $\mathrm{H}$ & 9.756153 & 9.129404 & 2.360717 & $\mathrm{H}$ & 6.620127 & 3.699512 & 2.005335 \\
\hline $\mathrm{H}$ & 9.661742 & 5.295379 & 2.341797 & $\mathrm{H}$ & 6.966739 & 3.962388 & 3.74122 \\
\hline $\mathrm{H}$ & 10.05772 & 6.717408 & 3.372456 & $\mathrm{H}$ & 5.671678 & 4.906357 & 2.94443 \\
\hline $\mathrm{H}$ & 8.385385 & 6.451945 & 2.809665 & $\mathrm{H}$ & 9.384044 & 4.308309 & 3.22166 \\
\hline $\mathrm{H}$ & 11.79663 & 7.619898 & 1.977773 & $\mathrm{H}$ & 9.109933 & 4.141553 & 1.45357 \\
\hline $\mathrm{H}$ & 11.71552 & 6.240585 & 0.836156 & $\mathrm{H}$ & 9.929642 & 5.595017 & 2.100848 \\
\hline $\mathrm{H}$ & 11.61407 & 7.927006 & 0.228217 & $\mathrm{C}$ & 9.017577 & 9.140503 & -0.9806 \\
\hline $\mathrm{C}$ & 9.741158 & 5.830979 & -3.7308 & $\mathrm{C}$ & 10.4274 & 8.504076 & -1.20126 \\
\hline $\mathrm{C}$ & 10.94699 & 4.857938 & -3.52435 & $\mathrm{C}$ & 8.58016 & 9.827186 & -2.3064 \\
\hline $\mathrm{C}$ & 8.839314 & 5.24814 & -4.8561 & $\mathrm{C}$ & 9.107086 & 10.23492 & 0.13123 \\
\hline $\mathrm{C}$ & 10.27011 & 7.225979 & -4.19453 & $\mathrm{H}$ & 10.80884 & 8.023214 & -0.2796 \\
\hline $\mathrm{H}$ & 11.65101 & 5.232365 & -2.75666 & $\mathrm{H}$ & 10.38409 & 7.731742 & -1.9946 \\
\hline $\mathrm{H}$ & 10.59157 & 3.858761 & -3.20248 & $\mathrm{H}$ & 11.15787 & 9.279488 & -1.51155 \\
\hline $\mathrm{H}$ & 11.50933 & 4.736049 & -4.47294 & $\mathrm{H}$ & 7.587949 & 10.31085 & -2.2076 \\
\hline $\mathrm{H}$ & 7.977081 & 5.909609 & -5.07402 & $\mathrm{H}$ & 9.313963 & 10.61224 & -2.5777 \\
\hline $\mathrm{H}$ & 9.428939 & 5.145098 & -5.7887 & $\mathrm{H}$ & 8.533771 & 9.105418 & -3.14616 \\
\hline $\mathrm{H}$ & 8.452169 & 4.245032 & -4.58758 & $\mathrm{H}$ & 9.806628 & 11.03912 & -0.1767 \\
\hline $\mathrm{H}$ & 10.82331 & 7.129103 & -5.15138 & $\mathrm{H}$ & 8.112698 & 10.68891 & 0.313583 \\
\hline $\mathrm{H}$ & 9.428783 & 7.930907 & -4.34796 & $\mathrm{H}$ & 9.470065 & 9.815733 & 1.08959 \\
\hline $\mathrm{H}$ & 10.95552 & 7.672147 & -3.44809 & $\mathrm{C}$ & 8.180066 & 0.544232 & 0.97393 \\
\hline $\mathrm{C}$ & 6.963248 & 2.40686 & 3.718377 & $\mathrm{C}$ & 8.973663 & 0.745733 & 2.3035 \\
\hline & 7.318539 & 3.658234 & 4.585228 & $\mathrm{C}$ & 6.672955 & 0.371049 & 1.3259 \\
\hline
\end{tabular}




\begin{tabular}{|c|c|c|c|c|c|c|c|}
\hline $\mathrm{C}$ & 5.422103 & 2.383282 & 3.501753 & $\mathrm{C}$ & 8.662651 & -0.77012 & 0.278271 \\
\hline $\mathrm{C}$ & 7.362909 & 1.110094 & 4.491512 & $\mathrm{H}$ & 10.06137 & 0.841604 & 2.121197 \\
\hline $\mathrm{H}$ & 8.403973 & 3.708133 & 4.798124 & $\mathrm{H}$ & 8.634826 & 1.659851 & 2.829601 \\
\hline $\mathrm{H}$ & 7.033641 & 4.590713 & 4.058825 & $\mathrm{H}$ & 8.819363 & -0.12347 & 2.973176 \\
\hline $\mathrm{H}$ & 6.776706 & 3.624021 & 5.552909 & $\mathrm{H}$ & 6.05191 & 0.197394 & 0.424335 \\
\hline $\mathrm{H}$ & 5.107048 & 1.516161 & 2.887164 & $\mathrm{H}$ & 6.55131 & -0.5122 & 1.983186 \\
\hline $\mathrm{H}$ & 4.912234 & 2.305218 & 4.482511 & $\mathrm{H}$ & 6.270298 & 1.253479 & 1.862948 \\
\hline $\mathrm{H}$ & 5.0616 & 3.308724 & 3.010346 & $\mathrm{H}$ & 8.495409 & -1.64212 & 0.939939 \\
\hline $\mathrm{H}$ & 6.81727 & 1.060568 & 5.455929 & $\mathrm{H}$ & 8.106753 & -0.9414 & -0.66557 \\
\hline $\mathrm{H}$ & 7.108661 & 0.206364 & 3.904687 & $\mathrm{H}$ & 9.741376 & -0.72442 & 0.031627 \\
\hline $\mathrm{H}$ & 8.446557 & 1.078236 & 4.715727 & $\mathrm{C}$ & 11.52099 & 3.479059 & -1.59087 \\
\hline $\mathrm{C}$ & 11.47328 & 2.462711 & 1.243919 & $\mathrm{C}$ & 12.26604 & 2.211276 & -2.11837 \\
\hline $\mathrm{C}$ & 11.83226 & 0.957384 & 1.468349 & $\mathrm{C}$ & 11.63899 & 4.594606 & -2.66862 \\
\hline $\mathrm{C}$ & 12.13538 & 2.942455 & -0.08095 & $\mathrm{C}$ & 12.20954 & 3.985928 & -0.28221 \\
\hline $\mathrm{C}$ & 12.06891 & 3.30794 & 2.413602 & $\mathrm{H}$ & 12.27416 & 1.395431 & -1.37018 \\
\hline $\mathrm{H}$ & 11.40441 & 0.580746 & 2.418418 & $\mathrm{H}$ & 11.78072 & 1.826058 & -3.03732 \\
\hline $\mathrm{H}$ & 11.43229 & 0.33252 & 0.646459 & $\mathrm{H}$ & 13.3198 & 2.460389 & -2.35952 \\
\hline $\mathrm{H}$ & 12.93341 & 0.82457 & 1.506492 & $\mathrm{H}$ & 11.16655 & 5.53808 & -2.33258 \\
\hline $\mathrm{H}$ & 11.84869 & 3.98533 & -0.32139 & $\mathrm{H}$ & 12.70865 & 4.806704 & -2.86683 \\
\hline $\mathrm{H}$ & 13.23847 & 2.900791 & 0.016811 & $\mathrm{H}$ & 11.17054 & 4.289464 & -3.62573 \\
\hline $\mathrm{H}$ & 11.85373 & 2.302 & -0.93954 & $\mathrm{H}$ & 13.2706 & 4.243273 & -0.4797 \\
\hline $\mathrm{H}$ & 13.17314 & 3.206045 & 2.425313 & $\mathrm{H}$ & 11.69582 & 4.889084 & 0.102572 \\
\hline $\mathrm{H}$ & 11.82329 & 4.380412 & 2.2973 & $\mathrm{H}$ & 12.1832 & 3.216369 & 0.513605 \\
\hline $\mathrm{H}$ & 11.69447 & 2.976933 & 3.401115 & $\mathrm{C}$ & 10.66894 & -4.78875 & 1.440555 \\
\hline $\mathrm{C}$ & 10.19239 & -6.26246 & -0.80324 & $\mathrm{C}$ & 11.14227 & -5.72151 & 2.599661 \\
\hline $\mathrm{C}$ & 10.61586 & -6.72031 & 0.630405 & $\mathrm{C}$ & 11.04569 & -5.46992 & 0.092281 \\
\hline $\mathrm{C}$ & 9.972708 & -7.52921 & -1.67903 & $\mathrm{C}$ & 11.42649 & -3.42457 & 1.53956 \\
\hline $\mathrm{C}$ & 11.34154 & -5.42279 & -1.44644 & $\mathrm{H}$ & 10.95471 & -5.27791 & 3.59606 \\
\hline $\mathrm{H}$ & 10.81455 & -5.85182 & 1.288156 & $\mathrm{H}$ & 10.62665 & -6.69965 & 2.559805 \\
\hline $\mathrm{H}$ & 9.813237 & -7.32405 & 1.098313 & $\mathrm{H}$ & 12.23313 & -5.90237 & 2.514629 \\
\hline $\mathrm{H}$ & 11.53685 & -7.33748 & 0.582877 & $\mathrm{H}$ & 10.84363 & -4.81017 & -0.77429 \\
\hline $\mathrm{H}$ & 9.659417 & -7.26291 & -2.70833 & $\mathrm{H}$ & 12.12829 & -5.70663 & 0.084784 \\
\hline $\mathrm{H}$ & 10.9187 & -8.10247 & -1.74846 & $\mathrm{H}$ & 10.48523 & -6.41452 & -0.05483 \\
\hline $\mathrm{H}$ & 9.206927 & -8.19975 & -1.24312 & $\mathrm{H}$ & 12.52236 & -3.58469 & 1.468459 \\
\hline $\mathrm{H}$ & 12.25425 & -6.04431 & -1.55183 & $\mathrm{H}$ & 11.11807 & -2.74646 & 0.720094 \\
\hline $\mathrm{H}$ & 11.04768 & -5.06275 & -2.45268 & $\mathrm{H}$ & 11.21265 & -2.91519 & 2.49992 \\
\hline $\mathrm{H}$ & 11.60492 & -4.54011 & -0.8325 & $\mathrm{C}$ & 6.623004 & -3.42029 & 4.303208 \\
\hline $\mathrm{C}$ & 7.93508 & -1.9323 & 0.795062 & $\mathrm{C}$ & 7.398247 & -2.20403 & 4.901631 \\
\hline $\mathrm{C}$ & 8.755429 & -1.02158 & -0.1757 & $\mathrm{C}$ & 5.113733 & -3.04585 & 4.237079 \\
\hline $\mathrm{C}$ & 6.551987 & -1.25616 & 1.031651 & $\mathrm{C}$ & 6.78068 & -4.65409 & 5.249915 \\
\hline $\mathrm{C}$ & 8.674837 & -2.02086 & 2.167002 & $\mathrm{H}$ & 8.48034 & -2.41335 & 5.005446 \\
\hline $\mathrm{H}$ & 9.767571 & -1.4348 & -0.3554 & $\mathrm{H}$ & 7.283551 & -1.31089 & 4.257053 \\
\hline $\mathrm{H}$ & 8.245707 & -0.93805 & -1.15682 & $\mathrm{H}$ & 7.002374 & -1.95611 & 5.907755 \\
\hline $\mathrm{H}$ & 8.862838 & -0.0045 & 0.247686 & $\mathrm{H}$ & 4.497106 & -3.88837 & 3.865834 \\
\hline $\mathrm{H}$ & 5.922499 & -1.8423 & 1.731166 & $\mathrm{H}$ & 4.751709 & -2.78685 & 5.251911 \\
\hline $\mathrm{H}$ & 6.707352 & -0.2509 & 1.467413 & $\mathrm{H}$ & 4.936649 & -2.16948 & 3.581779 \\
\hline $\mathrm{H}$ & 5.986764 & -1.11873 & 0.08812 & $\mathrm{H}$ & 6.378001 & -4.42049 & 6.257045 \\
\hline $\mathrm{H}$ & 8.787699 & -1.0074 & 2.600174 & $\mathrm{H}$ & 6.229421 & -5.52726 & 4.848411 \\
\hline
\end{tabular}




\begin{tabular}{|c|c|c|c|c|c|c|c|}
\hline $\mathrm{H}$ & 8.106826 & -2.64945 & 2.881252 & $\mathrm{H}$ & 7.842227 & -4.94773 & 5.361775 \\
\hline $\mathrm{H}$ & 9.687628 & -2.45569 & 2.062827 & $\mathrm{C}$ & -11.6581 & -3.56936 & -0.53485 \\
\hline $\mathrm{C}$ & -12.0332 & -4.17745 & -0.75756 & $\mathrm{C}$ & -12.45 & -4.65382 & -1.33282 \\
\hline $\mathrm{C}$ & -12.9692 & -5.29527 & -1.30141 & $\mathrm{C}$ & -11.8331 & -3.86726 & 0.982613 \\
\hline $\mathrm{C}$ & -12.0315 & -4.25499 & 0.802417 & $\mathrm{C}$ & -12.261 & -2.16091 & -0.84139 \\
\hline $\mathrm{C}$ & -12.6114 & -2.79371 & -1.20049 & $\mathrm{H}$ & -12.4268 & -4.46692 & -2.42335 \\
\hline $\mathrm{H}$ & -13.0714 & -5.24511 & -2.40366 & $\mathrm{H}$ & -12.0325 & -5.66308 & -1.15258 \\
\hline $\mathrm{H}$ & -12.6036 & -6.30592 & -1.03115 & $\mathrm{H}$ & -13.5126 & -4.65623 & -1.01534 \\
\hline $\mathrm{H}$ & -13.9812 & -5.17421 & -0.86647 & $\mathrm{H}$ & -11.3729 & -3.08225 & 1.614481 \\
\hline $\mathrm{H}$ & -11.4489 & -3.43204 & 1.256218 & $\mathrm{H}$ & -12.912 & -3.9072 & 1.232708 \\
\hline $\mathrm{H}$ & -13.0698 & -4.18809 & 1.187314 & $\mathrm{H}$ & -11.3792 & -4.83967 & 1.258464 \\
\hline $\mathrm{H}$ & -11.5889 & -5.2069 & 1.151185 & $\mathrm{H}$ & -13.3315 & -2.12514 & -0.55106 \\
\hline $\mathrm{H}$ & -13.6492 & -2.67026 & -0.82729 & $\mathrm{H}$ & -11.7205 & -1.37301 & -0.28139 \\
\hline $\mathrm{H}$ & -12.0009 & -1.95863 & -0.80497 & $\mathrm{H}$ & -12.1875 & -1.91883 & -1.91966 \\
\hline $\mathrm{H}$ & -12.6251 & -2.71102 & -2.30563 & $\mathrm{C}$ & -8.15956 & -3.49029 & -4.29228 \\
\hline $\mathrm{C}$ & -8.58023 & -6.29404 & -3.97449 & $\mathrm{C}$ & -8.7577 & -2.19181 & -4.92301 \\
\hline $\mathrm{C}$ & -9.49863 & -6.03911 & -5.21242 & $\mathrm{C}$ & -6.63141 & -3.51289 & -4.58487 \\
\hline $\mathrm{C}$ & -7.09695 & -6.20362 & -4.43574 & $\mathrm{C}$ & -8.80511 & -4.74497 & -4.96408 \\
\hline $\mathrm{C}$ & -8.82967 & -7.7367 & -3.42835 & $\mathrm{H}$ & -9.85269 & -2.12971 & -4.7694 \\
\hline $\mathrm{H}$ & -10.5704 & -6.1426 & -4.95451 & $\mathrm{H}$ & -8.29743 & -1.29171 & -4.47033 \\
\hline $\mathrm{H}$ & -9.34211 & -5.01678 & -5.61086 & $\mathrm{H}$ & -8.5632 & -2.16901 & -6.01479 \\
\hline $\mathrm{H}$ & -9.26925 & -6.76652 & -6.01818 & $\mathrm{H}$ & -6.14989 & -4.4258 & -4.18064 \\
\hline $\mathrm{H}$ & -6.39786 & -6.39357 & -3.59692 & $\mathrm{H}$ & -6.46331 & -3.50132 & -5.67997 \\
\hline $\mathrm{H}$ & -6.90372 & -6.96499 & -5.21739 & $\mathrm{H}$ & -6.11694 & -2.62905 & -4.15782 \\
\hline $\mathrm{H}$ & -6.86175 & -5.21045 & -4.86792 & $\mathrm{H}$ & -8.59357 & -4.74886 & -6.05294 \\
\hline $\mathrm{H}$ & -8.6091 & -8.48854 & -4.21385 & $\mathrm{H}$ & -8.39625 & -5.67697 & -4.52586 \\
\hline $\mathrm{H}$ & -8.17279 & -7.94195 & -2.55953 & $\mathrm{H}$ & -9.90379 & -4.76239 & -4.82917 \\
\hline $\mathrm{H}$ & -9.87847 & -7.87762 & -3.10358 & $\mathrm{Mn}$ & -0.19059 & 0.314839 & -1.39657 \\
\hline & & & & $\mathrm{O}$ & -0.60351 & -0.00254 & -3.33353 \\
\hline & & & & $\mathrm{O}$ & -0.54052 & 2.160631 & -4.03347 \\
\hline & & & & $\mathrm{C}$ & -0.70473 & 0.95273 & -4.24952 \\
\hline & & & & $\mathrm{C}$ & -1.05784 & 0.416614 & -5.65034 \\
\hline & & & & $\mathrm{H}$ & -1.11986 & 1.248262 & -6.37406 \\
\hline & & & & $\mathrm{H}$ & -2.02485 & -0.12316 & -5.60772 \\
\hline & & & & $\mathrm{H}$ & -0.28988 & -0.31376 & -5.97366 \\
\hline
\end{tabular}

1:ImC 60 LS state, doublet, $\langle\mathrm{S} 2\rangle=0.758038 \quad 2: \mathrm{ImC}_{60}$ quintet state $\langle\mathrm{S} 2\rangle=6.034694$

\begin{tabular}{lrrr|lrrr} 
Co & -1.89113 & 0.679028 & -0.57236 & Mn & -1.92754 & 0.451624 & -0.70286 \\
C & 0.527404 & 4.14724 & -0.29594 & $\mathrm{C}$ & 0.323833 & 4.0504 & -0.38724 \\
$\mathrm{C}$ & -0.70155 & 4.763296 & -0.646 & $\mathrm{C}$ & -0.92823 & 4.594097 & -0.78898 \\
$\mathrm{C}$ & -1.67367 & 3.669258 & -0.83105 & $\mathrm{C}$ & -1.81615 & 3.451361 & -1.05511 \\
$\mathrm{~N}$ & -2.93726 & 3.875238 & -1.21805 & $\mathrm{~N}$ & -3.0697 & 3.570951 & -1.51356 \\
$\mathrm{~N}$ & -1.10636 & -2.34219 & 0.84287 & $\mathrm{~N}$ & -0.89613 & -2.60818 & 0.417743 \\
$\mathrm{C}$ & -2.32435 & -2.1633 & 0.322441 & $\mathrm{C}$ & -2.10979 & -2.4977 & -0.13426 \\
$\mathrm{C}$ & -3.38149 & -3.19139 & 0.380852 & $\mathrm{C}$ & -3.12808 & -3.56006 & -0.07365 \\
$\mathrm{C}$ & -4.49418 & -2.65493 & -0.31868 & $\mathrm{C}$ & -4.27653 & -3.06107 & -0.74842 \\
$\mathrm{C}$ & -4.10884 & -1.30816 & -0.7532 & $\mathrm{C}$ & -3.94934 & -1.7052 & -1.20291 \\
$\mathrm{C}$ & -5.17396 & 3.046832 & -1.88375 & $\mathrm{C}$ & -5.21044 & 2.623013 & -2.32662
\end{tabular}




\begin{tabular}{|c|c|c|c|c|c|c|c|}
\hline $\mathrm{C}$ & -3.78857 & 2.862636 & -1.40792 & $\mathrm{C}$ & -3.84786 & 2.516776 & -1.77907 \\
\hline $\mathrm{N}$ & -2.81411 & -1.03536 & -0.33313 & $\mathrm{~N}$ & -2.65254 & -1.40489 & -0.80178 \\
\hline $\mathrm{C}$ & 1.861478 & -0.33139 & 1.113216 & $\mathrm{C}$ & 1.923784 & -0.40772 & 0.884304 \\
\hline $\mathrm{C}$ & 1.17954 & -1.55061 & 1.363531 & $\mathrm{C}$ & 1.323486 & -1.68732 & 1.051057 \\
\hline $\mathrm{C}$ & -0.17494 & -1.38358 & 0.805413 & $\mathrm{C}$ & -0.01905 & -1.59611 & 0.454906 \\
\hline $\mathrm{C}$ & -5.71199 & 1.743826 & -2.03751 & $\mathrm{C}$ & -5.67621 & 1.292586 & -2.51266 \\
\hline $\mathrm{N}$ & -0.30189 & -0.09919 & 0.273164 & $\mathrm{~N}$ & -0.20791 & -0.2962 & -0.01498 \\
\hline $\mathrm{C}$ & -4.69218 & 0.804476 & -1.56222 & $\mathrm{C}$ & -4.6332 & 0.394839 & -2.00027 \\
\hline $\mathrm{N}$ & -4.95886 & -0.49102 & -1.38107 & $\mathrm{~N}$ & -4.81791 & -0.91764 & -1.84537 \\
\hline $\mathrm{C}$ & 0.268871 & 2.704579 & -0.22507 & $\mathrm{C}$ & 0.168492 & 2.593157 & -0.36884 \\
\hline $\mathrm{C}$ & -0.81327 & 6.164109 & -0.68339 & $\mathrm{C}$ & -1.13175 & 5.985548 & -0.79727 \\
\hline $\mathrm{C}$ & -3.46479 & -4.4343 & 1.027834 & $\mathrm{C}$ & -3.15432 & -4.7845 & 0.605641 \\
\hline $\mathrm{C}$ & -5.9794 & 4.18266 & -2.09936 & $\mathrm{C}$ & -6.05395 & 3.724023 & -2.56664 \\
\hline $\mathrm{C}$ & -7.03145 & 1.528534 & -2.46321 & $\mathrm{C}$ & -6.97316 & 1.026485 & -2.97763 \\
\hline $\mathrm{C}$ & 1.674798 & 4.904133 & -0.01902 & $\mathrm{C}$ & 1.401364 & 4.877308 & -0.03568 \\
\hline $\mathrm{C}$ & -5.71463 & -3.3457 & -0.37281 & $\mathrm{C}$ & -5.49005 & -3.76355 & -0.70138 \\
\hline $\mathrm{C}$ & -5.81922 & -4.57206 & 0.301538 & $\mathrm{C}$ & -5.54662 & -4.95748 & 0.036477 \\
\hline $\mathrm{C}$ & -4.69775 & -5.1246 & 1.007826 & $\mathrm{C}$ & -4.37761 & -5.48941 & 0.678277 \\
\hline $\mathrm{C}$ & 1.805306 & -2.58646 & 2.082139 & $\mathrm{C}$ & 1.993811 & -2.70263 & 1.761594 \\
\hline $\mathrm{C}$ & 3.10883 & -2.36666 & 2.569043 & $\mathrm{C}$ & 3.247207 & -2.40468 & 2.328468 \\
\hline $\mathrm{C}$ & 3.78342 & -1.1218 & 2.323773 & $\mathrm{C}$ & 3.838899 & -1.10238 & 2.162096 \\
\hline $\mathrm{C}$ & 3.172044 & -0.11309 & 1.562795 & $\mathrm{C}$ & 3.194413 & -0.11536 & 1.403147 \\
\hline $\mathrm{C}$ & 1.579219 & 6.30559 & -0.07018 & $\mathrm{C}$ & 1.211862 & 6.269821 & -0.05417 \\
\hline $\mathrm{C}$ & 0.321753 & 6.937097 & -0.36892 & $\mathrm{C}$ & -0.07277 & 6.822736 & -0.40097 \\
\hline $\mathrm{C}$ & -7.84939 & 2.653824 & -2.64659 & $\mathrm{C}$ & -7.83808 & 2.11739 & -3.16329 \\
\hline $\mathrm{C}$ & -7.32626 & 3.977512 & -2.45266 & $\mathrm{C}$ & -7.3785 & 3.462373 & -2.95927 \\
\hline $\mathrm{N}$ & -3.54464 & 1.503433 & -1.21254 & $\mathrm{~N}$ & -3.55026 & 1.167773 & -1.59852 \\
\hline $\mathrm{N}$ & 1.195135 & 1.839753 & 0.192816 & $\mathrm{~N}$ & 1.12587 & 1.765051 & 0.058337 \\
\hline $\mathrm{N}$ & -1.05704 & 2.451595 & -0.55173 & $\mathrm{~N}$ & -1.12306 & 2.278718 & -0.772 \\
\hline $\mathrm{C}$ & 0.906948 & 0.561721 & 0.45168 & $\mathrm{C}$ & 0.935351 & 0.45414 & 0.238485 \\
\hline $\mathrm{H}$ & 1.30734 & -3.54139 & 2.288754 & $\mathrm{H}$ & 1.558476 & -3.69768 & 1.912158 \\
\hline $\mathrm{H}$ & 3.695737 & 0.823741 & 1.345065 & $\mathrm{H}$ & 3.653395 & 0.866364 & 1.247247 \\
\hline $\mathrm{H}$ & 2.630936 & 4.437437 & 0.246691 & $\mathrm{H}$ & 2.373657 & 4.469727 & 0.265941 \\
\hline $\mathrm{H}$ & -1.75941 & 6.670047 & -0.91117 & $\mathrm{H}$ & -2.09788 & 6.433202 & -1.05996 \\
\hline $\mathrm{H}$ & -5.6039 & 5.203664 & -1.96034 & $\mathrm{H}$ & -5.72719 & 4.758144 & -2.40473 \\
\hline $\mathrm{H}$ & -7.41449 & 0.514653 & -2.61904 & $\mathrm{H}$ & -7.31495 & -0.00108 & -3.14104 \\
\hline $\mathrm{H}$ & -6.59412 & -2.93581 & -0.88415 & $\mathrm{H}$ & -6.41008 & -3.36546 & -1.14567 \\
\hline $\mathrm{H}$ & -2.6245 & -4.85671 & 1.592094 & $\mathrm{H}$ & -2.28176 & -5.16982 & 1.146642 \\
\hline $\mathrm{C}$ & -9.3822 & 5.158706 & -2.038 & $\mathrm{C}$ & -9.46342 & 4.572083 & -2.51688 \\
\hline $\mathrm{C}$ & -10.3827 & 5.86402 & -2.72499 & $\mathrm{C}$ & -10.5079 & 5.2326 & -3.18233 \\
\hline $\mathrm{C}$ & -9.61228 & 4.584658 & -0.77666 & $\mathrm{C}$ & -9.62948 & 4.017488 & -1.23743 \\
\hline $\mathrm{C}$ & -11.6654 & 5.99377 & -2.15082 & $\mathrm{C}$ & -11.7707 & 5.340873 & -2.56194 \\
\hline $\mathrm{H}$ & -10.1326 & 6.281608 & -3.70581 & $\mathrm{H}$ & -10.3053 & 5.636822 & -4.17962 \\
\hline $\mathrm{C}$ & -10.8944 & 4.681072 & -0.1926 & $\mathrm{C}$ & -10.8899 & 4.096462 & -0.60489 \\
\hline $\mathrm{H}$ & -8.7978 & 4.050078 & -0.2774 & $\mathrm{H}$ & -8.78242 & 3.518077 & -0.7564 \\
\hline $\mathrm{C}$ & -11.8982 & 5.389284 & -0.89235 & $\mathrm{C}$ & -11.938 & 4.761302 & -1.28134 \\
\hline $\mathrm{H}$ & -12.8957 & 5.469529 & -0.44286 & $\mathrm{H}$ & -12.9184 & 4.829073 & -0.79377 \\
\hline $\mathrm{C}$ & -10.073 & 1.679224 & -2.44564 & $\mathrm{C}$ & -10.0133 & 1.091193 & -2.86384 \\
\hline
\end{tabular}




\begin{tabular}{|c|c|c|c|c|c|c|c|}
\hline $\mathrm{C}$ & -11.3565 & 1.671746 & -3.01976 & $\mathrm{C}$ & -11.3227 & 1.023631 & -3.36903 \\
\hline $\mathrm{C}$ & -9.76316 & 0.866985 & -1.33987 & $\mathrm{C}$ & -9.62328 & 0.344083 & -1.74013 \\
\hline $\mathrm{C}$ & -12.3544 & 0.820874 & -2.50262 & $\mathrm{C}$ & -12.268 & 0.174231 & -2.75654 \\
\hline $\mathrm{H}$ & -11.5404 & 2.348943 & -3.85979 & $\mathrm{H}$ & -11.569 & 1.653072 & -4.22989 \\
\hline $\mathrm{C}$ & -10.7319 & -0.03075 & -0.8359 & $\mathrm{C}$ & -10.5353 & -0.55246 & -1.14229 \\
\hline $\mathrm{H}$ & -8.77448 & 0.916978 & -0.88243 & $\mathrm{H}$ & -8.61559 & 0.440345 & -1.33609 \\
\hline $\mathrm{C}$ & -12.0169 & -0.03214 & -1.42279 & $\mathrm{C}$ & -11.8504 & -0.61438 & -1.65536 \\
\hline $\mathrm{H}$ & -12.7773 & -0.71805 & -1.03111 & $\mathrm{H}$ & -12.5686 & -1.29816 & -1.18649 \\
\hline $\mathrm{C}$ & 0.58149 & 9.124289 & 0.646416 & $\mathrm{C}$ & -0.06585 & 8.975446 & 0.713638 \\
\hline $\mathrm{C}$ & 0.808009 & 8.608108 & 1.935294 & $\mathrm{C}$ & 0.131228 & 8.419109 & 1.990403 \\
\hline $\mathrm{C}$ & 0.742575 & 10.48461 & 0.359412 & $\mathrm{C}$ & -0.03405 & 10.35806 & 0.499603 \\
\hline $\mathrm{C}$ & 1.264712 & 9.466377 & 2.953544 & $\mathrm{C}$ & 0.420654 & 9.267098 & 3.076581 \\
\hline $\mathrm{H}$ & 0.652483 & 7.541742 & 2.118562 & $\mathrm{H}$ & 0.079089 & 7.334599 & 2.11662 \\
\hline $\mathrm{C}$ & 1.157642 & 11.37497 & 1.376887 & $\mathrm{C}$ & 0.21526 & 11.23307 & 1.582436 \\
\hline $\mathrm{H}$ & 0.549896 & 10.82228 & -0.6655 & $\mathrm{H}$ & -0.19682 & 10.7269 & -0.51953 \\
\hline $\mathrm{C}$ & 1.431254 & 10.84357 & 2.655987 & $\mathrm{C}$ & 0.456392 & 10.66781 & 2.853706 \\
\hline $\mathrm{H}$ & 1.784737 & 11.51163 & 3.447766 & $\mathrm{H}$ & 0.679285 & 11.32786 & 3.698254 \\
\hline $\mathrm{C}$ & 3.234653 & 8.077709 & -0.40168 & $\mathrm{C}$ & 2.719899 & 8.190672 & -0.22366 \\
\hline $\mathrm{C}$ & 4.17594 & 8.855451 & 0.283485 & $\mathrm{C}$ & 3.523914 & 9.028076 & 0.558218 \\
\hline $\mathrm{C}$ & 2.873074 & 8.353354 & -1.73247 & $\mathrm{C}$ & 2.415898 & 8.491443 & -1.56291 \\
\hline $\mathrm{C}$ & 4.787914 & 9.954614 & -0.36027 & $\mathrm{C}$ & 4.041964 & 10.2228 & 0.00848 \\
\hline $\mathrm{H}$ & 4.413703 & 8.580861 & 1.317672 & $\mathrm{H}$ & 3.724133 & 8.728301 & 1.593178 \\
\hline $\mathrm{C}$ & 3.442828 & 9.46567 & -2.38356 & $\mathrm{C}$ & 2.892271 & 9.693479 & -2.12285 \\
\hline $\mathrm{H}$ & 2.143464 & 7.711925 & -2.23386 & $\mathrm{H}$ & 1.79982 & 7.79765 & -2.14135 \\
\hline $\mathrm{C}$ & 4.396695 & 10.24973 & -1.6844 & $\mathrm{C}$ & 3.699889 & 10.54304 & -1.32327 \\
\hline $\mathrm{H}$ & 4.840227 & 11.1113 & -2.1941 & $\mathrm{H}$ & 4.069537 & 11.47654 & -1.76034 \\
\hline $\mathrm{C}$ & 5.54371 & 0.166204 & 3.405779 & $\mathrm{C}$ & 5.430161 & 0.290547 & 3.36772 \\
\hline $\mathrm{C}$ & 4.701371 & 1.2092 & 3.830405 & $\mathrm{C}$ & 4.487628 & 1.254325 & 3.766837 \\
\hline $\mathrm{C}$ & 6.934803 & 0.221552 & 3.600954 & $\mathrm{C}$ & 6.801397 & 0.450421 & 3.630677 \\
\hline $\mathrm{C}$ & 5.264437 & 2.373167 & 4.401988 & $\mathrm{C}$ & 4.92707 & 2.44656 & 4.385791 \\
\hline $\mathrm{H}$ & 3.620775 & 1.122342 & 3.702491 & $\mathrm{H}$ & 3.424656 & 1.084816 & 3.5862 \\
\hline $\mathrm{C}$ & 7.517486 & 1.364947 & 4.183858 & $\mathrm{C}$ & 7.261199 & 1.622226 & 4.265565 \\
\hline $\mathrm{H}$ & 7.528166 & -0.64241 & 3.290749 & $\mathrm{H}$ & 7.476285 & -0.35683 & 3.334176 \\
\hline $\mathrm{C}$ & 6.667102 & 2.433108 & 4.561369 & $\mathrm{C}$ & 6.310821 & 2.610939 & 4.620707 \\
\hline $\mathrm{H}$ & 7.112677 & 3.335399 & 4.997479 & $\mathrm{H}$ & 6.661095 & 3.533872 & 5.099501 \\
\hline $\mathrm{C}$ & 4.559835 & -3.21624 & 4.316554 & $\mathrm{C}$ & 4.643069 & -3.18846 & 4.145668 \\
\hline $\mathrm{C}$ & 4.224035 & -2.28664 & 5.313237 & $\mathrm{C}$ & 4.165712 & -2.31423 & 5.134892 \\
\hline $\mathrm{C}$ & 5.717198 & -4.00322 & 4.39086 & $\mathrm{C}$ & 5.855441 & -3.87798 & 4.28002 \\
\hline $\mathrm{C}$ & 5.106905 & -2.08467 & 6.395526 & $\mathrm{C}$ & 4.959413 & -2.06186 & 6.274248 \\
\hline $\mathrm{H}$ & 3.301926 & -1.70475 & 5.210385 & $\mathrm{H}$ & 3.203977 & -1.81251 & 4.983265 \\
\hline $\mathrm{C}$ & 6.607086 & -3.83404 & 5.473626 & $\mathrm{C}$ & 6.659399 & -3.65645 & 5.418903 \\
\hline $\mathrm{H}$ & 5.907359 & -4.71208 & 3.579491 & $\mathrm{H}$ & 6.156777 & -4.55062 & 3.471897 \\
\hline $\mathrm{C}$ & 6.288493 & -2.86204 & 6.451282 & $\mathrm{C}$ & 6.1982 & -2.73753 & 6.39036 \\
\hline $\mathrm{H}$ & 6.985779 & -2.69831 & 7.281927 & $\mathrm{H}$ & 6.827179 & -2.53379 & 7.26514 \\
\hline $\mathrm{C}$ & -5.67674 & -7.29426 & 1.608473 & $\mathrm{C}$ & -5.37236 & -7.56501 & 1.557461 \\
\hline $\mathrm{C}$ & -6.32733 & -7.7586 & 2.754205 & $\mathrm{C}$ & -5.87611 & -7.83739 & 2.830238 \\
\hline $\mathrm{C}$ & -5.90118 & -7.85901 & 0.340971 & $\mathrm{C}$ & -5.81403 & -8.25133 & 0.413523 \\
\hline $\mathrm{C}$ & -7.27141 & -8.8083 & 2.651928 & $\mathrm{C}$ & -6.88949 & -8.81301 & 2.99275 \\
\hline
\end{tabular}




\begin{tabular}{|c|c|c|c|c|c|c|c|}
\hline $\mathrm{H}$ & -6.10196 & -7.26799 & 3.707483 & $\mathrm{H}$ & -5.4821 & -7.25962 & 3.673704 \\
\hline $\mathrm{C}$ & -6.85214 & -8.88788 & 0.206906 & $\mathrm{C}$ & -6.83618 & -9.2099 & 0.542301 \\
\hline $\mathrm{H}$ & -5.36564 & -7.4517 & -0.52246 & $\mathrm{H}$ & -5.38209 & -7.99262 & -0.55836 \\
\hline $\mathrm{C}$ & -7.52111 & -9.3478 & 1.373194 & $\mathrm{C}$ & -7.35657 & -9.47459 & 1.838641 \\
\hline $\mathrm{H}$ & -8.26337 & -10.1457 & 1.267167 & $\mathrm{H}$ & -8.15328 & -10.2196 & 1.936481 \\
\hline $\mathrm{C}$ & -7.95042 & -5.07674 & 1.305311 & $\mathrm{C}$ & -7.55697 & -5.23009 & 1.301541 \\
\hline $\mathrm{C}$ & -9.15565 & -5.78889 & 1.177915 & $\mathrm{C}$ & -8.78767 & -5.89654 & 1.430029 \\
\hline $\mathrm{C}$ & -7.69099 & -4.25466 & 2.41429 & $\mathrm{C}$ & -7.13765 & -4.2646 & 2.231469 \\
\hline $\mathrm{C}$ & -10.1594 & -5.64991 & 2.158049 & $\mathrm{C}$ & -9.65557 & -5.56292 & 2.491376 \\
\hline $\mathrm{H}$ & -9.27479 & -6.4307 & 0.299921 & $\mathrm{H}$ & -9.03913 & -6.65609 & 0.68389 \\
\hline $\mathrm{C}$ & -8.67345 & -4.11809 & 3.422213 & $\mathrm{C}$ & -7.98145 & -3.92818 & 3.313838 \\
\hline $\mathrm{H}$ & -6.73529 & -3.72724 & 2.478369 & $\mathrm{H}$ & -6.17534 & -3.76411 & 2.098849 \\
\hline $\mathrm{C}$ & -9.89831 & -4.80716 & 3.267372 & $\mathrm{C}$ & -9.23473 & -4.57478 & 3.41638 \\
\hline $\mathrm{H}$ & -10.6694 & -4.69161 & 4.038007 & $\mathrm{H}$ & -9.90218 & -4.30449 & 4.243207 \\
\hline $\mathrm{O}$ & 3.734465 & -3.4365 & 3.194317 & $\mathrm{O}$ & 3.914273 & -3.44176 & 2.962842 \\
\hline $\mathrm{O}$ & 5.088351 & -1.00992 & 2.788057 & $\mathrm{O}$ & 5.096612 & -0.90818 & 2.713349 \\
\hline $\mathrm{O}$ & 2.717192 & 6.997789 & 0.330067 & $\mathrm{O}$ & 2.278405 & 7.022007 & 0.419811 \\
\hline $\mathrm{O}$ & 0.168656 & 8.32004 & -0.43188 & $\mathrm{O}$ & -0.3222 & 8.191432 & -0.42684 \\
\hline $\mathrm{O}$ & -8.12462 & 5.094865 & -2.6715 & $\mathrm{O}$ & -8.22275 & 4.540285 & -3.1886 \\
\hline $\mathrm{O}$ & -9.17078 & 2.56476 & -3.0623 & $\mathrm{O}$ & -9.15667 & 1.965348 & -3.56506 \\
\hline $\mathrm{O}$ & -7.04926 & -5.23789 & 0.245184 & $\mathrm{O}$ & -6.79184 & -5.57963 & 0.173883 \\
\hline $\mathrm{O}$ & -4.74589 & -6.25445 & 1.809008 & $\mathrm{O}$ & -4.33986 & -6.60375 & 1.49409 \\
\hline $\mathrm{C}$ & 1.610787 & 8.933814 & 4.372121 & $\mathrm{C}$ & 0.722986 & 8.700567 & 4.49216 \\
\hline $\mathrm{C}$ & 0.759338 & 9.688389 & 5.442062 & $\mathrm{C}$ & -0.27358 & 9.310791 & 5.528173 \\
\hline $\mathrm{C}$ & 1.334908 & 7.408455 & 4.51166 & $\mathrm{C}$ & 0.596746 & 7.150129 & 4.545391 \\
\hline $\mathrm{C}$ & 3.130192 & 9.177346 & 4.646159 & $\mathrm{C}$ & 2.187006 & 9.080043 & 4.886053 \\
\hline $\mathrm{H}$ & 0.956749 & 10.77777 & 5.428892 & $\mathrm{H}$ & -0.19743 & 10.41446 & 5.569483 \\
\hline $\mathrm{H}$ & -0.32291 & 9.536386 & 5.257967 & $\mathrm{H}$ & -1.31817 & 9.051301 & 5.263945 \\
\hline $\mathrm{H}$ & 0.996238 & 9.31155 & 6.458456 & $\mathrm{H}$ & -0.0626 & 8.9163 & 6.543436 \\
\hline $\mathrm{H}$ & 1.936317 & 6.816971 & 3.793343 & $\mathrm{H}$ & 1.304675 & 6.65887 & 3.848942 \\
\hline $\mathrm{H}$ & 1.604336 & 7.075334 & 5.534101 & $\mathrm{H}$ & 0.828207 & 6.794603 & 5.569565 \\
\hline $\mathrm{H}$ & 0.264884 & 7.168551 & 4.35038 & $\mathrm{H}$ & -0.42886 & 6.812451 & 4.295708 \\
\hline $\mathrm{H}$ & 3.404771 & 8.809695 & 5.656517 & $\mathrm{H}$ & 2.426554 & 8.693389 & 5.898103 \\
\hline $\mathrm{H}$ & 3.74925 & 8.638192 & 3.901424 & $\mathrm{H}$ & 2.907296 & 8.640247 & 4.167451 \\
\hline $\mathrm{H}$ & 3.388179 & 10.25241 & 4.589544 & $\mathrm{H}$ & 2.338348 & 10.17661 & 4.891411 \\
\hline $\mathrm{C}$ & 1.266476 & 12.89205 & 1.057571 & $\mathrm{C}$ & 0.184137 & 12.7672 & 1.33661 \\
\hline $\mathrm{C}$ & 1.860774 & 13.70766 & 2.242073 & $\mathrm{C}$ & 0.632471 & 13.57811 & 2.58704 \\
\hline $\mathrm{C}$ & 2.17171 & 13.1129 & -0.19561 & $\mathrm{C}$ & 1.126972 & 13.13759 & 0.148278 \\
\hline $\mathrm{C}$ & -0.16736 & 13.43889 & 0.757952 & $\mathrm{C}$ & -1.2802 & 13.18272 & 0.979597 \\
\hline $\mathrm{H}$ & 1.219304 & 13.64892 & 3.143792 & $\mathrm{H}$ & -0.04197 & 13.40939 & 3.449961 \\
\hline $\mathrm{H}$ & 2.87653 & 13.35854 & 2.514421 & $\mathrm{H}$ & 1.664837 & 13.32019 & 2.896636 \\
\hline $\mathrm{H}$ & 1.936346 & 14.7752 & 1.954169 & $\mathrm{H}$ & 0.610424 & 14.66111 & 2.352711 \\
\hline $\mathrm{H}$ & 1.748452 & 12.635 & -1.0981 & $\mathrm{H}$ & 0.807349 & 12.66129 & -0.79696 \\
\hline $\mathrm{H}$ & 2.277093 & 14.19762 & -0.4037 & $\mathrm{H}$ & 1.129624 & 14.23579 & -0.009 \\
\hline $\mathrm{H}$ & 3.17935 & 12.6846 & -0.03854 & $\mathrm{H}$ & 2.162947 & 12.80746 & 0.34989 \\
\hline $\mathrm{H}$ & -0.12697 & 14.52334 & 0.525553 & $\mathrm{H}$ & -1.33683 & 14.27484 & 0.7911 \\
\hline $\mathrm{H}$ & -0.62398 & 12.91788 & -0.10597 & $\mathrm{H}$ & -1.63623 & 12.65671 & 0.072342 \\
\hline $\mathrm{H}$ & -0.83229 & 13.29313 & 1.632672 & $\mathrm{H}$ & -1.97193 & 12.93509 & 1.809491 \\
\hline
\end{tabular}




\begin{tabular}{|c|c|c|c|c|c|c|c|}
\hline $\mathrm{C}$ & -11.2299 & 4.030011 & 1.17725 & $\mathrm{C}$ & -11.1504 & 3.478019 & 0.796097 \\
\hline $\mathrm{C}$ & -12.4094 & 3.022457 & 0.988082 & $\mathrm{C}$ & -12.311 & 2.437807 & 0.684475 \\
\hline $\mathrm{C}$ & -10.02 & 3.253824 & 1.772115 & $\mathrm{C}$ & -9.89656 & 2.750668 & 1.360732 \\
\hline $\mathrm{C}$ & -11.6461 & 5.143618 & 2.190497 & $\mathrm{C}$ & -11.553 & 4.611 & 1.793258 \\
\hline $\mathrm{H}$ & -13.3266 & 3.530689 & 0.632573 & $\mathrm{H}$ & -13.2544 & 2.913882 & 0.353577 \\
\hline $\mathrm{H}$ & -12.1451 & 2.241212 & 0.251285 & $\mathrm{H}$ & -12.0568 & 1.643574 & -0.04166 \\
\hline $\mathrm{H}$ & -12.649 & 2.528821 & 1.952745 & $\mathrm{H}$ & -12.4979 & 1.964693 & 1.670607 \\
\hline $\mathrm{H}$ & -9.15712 & 3.922391 & 1.965666 & $\mathrm{H}$ & -9.04326 & 3.44522 & 1.495672 \\
\hline $\mathrm{H}$ & -10.3124 & 2.795066 & 2.737924 & $\mathrm{H}$ & -10.1339 & 2.316468 & 2.352454 \\
\hline $\mathrm{H}$ & -9.69105 & 2.441024 & 1.096726 & $\mathrm{H}$ & -9.57505 & 1.924049 & 0.699205 \\
\hline $\mathrm{H}$ & -11.8911 & 4.695447 & 3.175657 & $\mathrm{H}$ & -11.7505 & 4.184797 & 2.7985 \\
\hline $\mathrm{H}$ & -10.822 & 5.871057 & 2.333919 & $\mathrm{H}$ & -10.7403 & 5.359156 & 1.885628 \\
\hline $\mathrm{H}$ & -12.5353 & 5.702127 & 1.838748 & $\mathrm{H}$ & -12.467 & 5.140678 & 1.460749 \\
\hline $\mathrm{C}$ & -12.814 & 6.767189 & -2.85554 & $\mathrm{C}$ & -12.9677 & 6.064885 & -3.2378 \\
\hline $\mathrm{C}$ & -13.2529 & 7.965091 & -1.95287 & $\mathrm{C}$ & -13.4203 & 7.258422 & -2.33616 \\
\hline $\mathrm{C}$ & -12.3828 & 7.331347 & -4.23973 & $\mathrm{C}$ & -12.6019 & 6.625631 & -4.64199 \\
\hline $\mathrm{C}$ & -14.0289 & 5.810388 & -3.07656 & $\mathrm{C}$ & -14.1531 & 5.061493 & -3.40663 \\
\hline $\mathrm{H}$ & -13.6141 & 7.619141 & -0.96519 & $\mathrm{H}$ & -13.739 & 6.913371 & -1.33368 \\
\hline $\mathrm{H}$ & -12.4037 & 8.65617 & -1.78097 & $\mathrm{H}$ & -12.5912 & 7.980835 & -2.19896 \\
\hline $\mathrm{H}$ & -14.073 & 8.532932 & -2.43877 & $\mathrm{H}$ & -14.2744 & 7.790995 & -2.80322 \\
\hline $\mathrm{H}$ & -12.0801 & 6.52262 & -4.93458 & $\mathrm{H}$ & -12.2948 & 5.819031 & -5.33737 \\
\hline $\mathrm{H}$ & -13.2324 & 7.873426 & -4.70108 & $\mathrm{H}$ & -13.4841 & 7.132937 & -5.08125 \\
\hline $\mathrm{H}$ & -11.539 & 8.043566 & -4.14605 & $\mathrm{H}$ & -11.7803 & 7.367155 & -4.58425 \\
\hline $\mathrm{H}$ & -14.8622 & 6.35307 & -3.56863 & $\mathrm{H}$ & -15.0239 & 5.567451 & -3.87203 \\
\hline $\mathrm{H}$ & -13.7408 & 4.957749 & -3.72255 & $\mathrm{H}$ & -13.8561 & 4.214636 & -4.05609 \\
\hline $\mathrm{H}$ & -14.4073 & 5.397977 & -2.12151 & $\mathrm{H}$ & -14.4797 & 4.643486 & -2.43499 \\
\hline $\mathrm{C}$ & 4.37944 & 3.57506 & 4.838248 & $\mathrm{C}$ & 3.928519 & 3.567559 & 4.790968 \\
\hline $\mathrm{C}$ & 4.79487 & 4.842184 & 4.021453 & $\mathrm{C}$ & 4.295308 & 4.88195 & 4.0272 \\
\hline $\mathrm{C}$ & 2.863978 & 3.317843 & 4.590365 & $\mathrm{C}$ & 2.454515 & 3.205086 & 4.444722 \\
\hline $\mathrm{C}$ & 4.587681 & 3.845443 & 6.363104 & $\mathrm{C}$ & 4.024804 & 3.814746 & 6.330701 \\
\hline $\mathrm{H}$ & 5.862691 & 5.093445 & 4.171302 & $\mathrm{H}$ & 5.327406 & 5.212957 & 4.253382 \\
\hline $\mathrm{H}$ & 4.630372 & 4.682703 & 2.936966 & $\mathrm{H}$ & 4.215632 & 4.736097 & 2.931294 \\
\hline $\mathrm{H}$ & 4.189524 & 5.716139 & 4.33694 & $\mathrm{H}$ & 3.602562 & 5.697982 & 4.317229 \\
\hline $\mathrm{H}$ & 2.495743 & 2.446723 & 5.16884 & $\mathrm{H}$ & 2.114329 & 2.300922 & 4.988317 \\
\hline $\mathrm{H}$ & 2.282175 & 4.204131 & 4.912204 & $\mathrm{H}$ & 1.790002 & 4.040975 & 4.739551 \\
\hline $\mathrm{H}$ & 2.642973 & 3.144537 & 3.518002 & $\mathrm{H}$ & 2.312589 & 3.033644 & 3.358732 \\
\hline $\mathrm{H}$ & 3.963251 & 4.702776 & 6.688566 & $\mathrm{H}$ & 3.318827 & 4.61467 & 6.634699 \\
\hline $\mathrm{H}$ & 4.300784 & 2.958218 & 6.961245 & $\mathrm{H}$ & 3.770961 & 2.893851 & 6.89161 \\
\hline $\mathrm{H}$ & 5.643429 & 4.084735 & 6.595892 & $\mathrm{H}$ & 5.044018 & 4.125935 & 6.631455 \\
\hline $\mathrm{C}$ & 9.049647 & 1.483273 & 4.408249 & $\mathrm{C}$ & 8.765697 & 1.85523 & 4.572072 \\
\hline $\mathrm{C}$ & 9.333525 & 1.699454 & 5.929594 & $\mathrm{C}$ & 8.955965 & 2.020858 & 6.11421 \\
\hline $\mathrm{C}$ & 9.812423 & 0.208871 & 3.946125 & $\mathrm{C}$ & 9.654378 & 0.670952 & 4.095585 \\
\hline $\mathrm{C}$ & 9.593332 & 2.700805 & 3.593512 & $\mathrm{C}$ & 9.244653 & 3.149988 & 3.840121 \\
\hline $\mathrm{H}$ & 8.847897 & 2.618368 & 6.311494 & $\mathrm{H}$ & 8.377195 & 2.878614 & 6.508196 \\
\hline $\mathrm{H}$ & 8.952789 & 0.844345 & 6.522153 & $\mathrm{H}$ & 8.619103 & 1.110327 & 6.648279 \\
\hline $\mathrm{H}$ & 10.42493 & 1.790022 & 6.106728 & $\mathrm{H}$ & 10.02603 & 2.190712 & 6.352759 \\
\hline $\mathrm{H}$ & 9.679389 & 0.02151 & 2.862905 & $\mathrm{H}$ & 9.585718 & 0.52048 & 3.000703 \\
\hline $\mathrm{H}$ & 10.89721 & 0.337525 & 4.133897 & $\mathrm{H}$ & 10.71472 & 0.88182 & 4.339623 \\
\hline
\end{tabular}




\begin{tabular}{|c|c|c|c|c|c|c|c|}
\hline $\mathrm{H}$ & 9.478519 & -0.69134 & 4.497772 & $\mathrm{H}$ & 9.37595 & -0.27758 & 4.594418 \\
\hline $\mathrm{H}$ & 10.68838 & 2.802495 & 3.739892 & $\mathrm{H}$ & 10.31986 & 3.330988 & 4.045148 \\
\hline $\mathrm{H}$ & 9.398311 & 2.563655 & 2.511049 & $\mathrm{H}$ & 9.1115 & 3.049902 & 2.744326 \\
\hline $\mathrm{H}$ & 9.117133 & 3.649728 & 3.907871 & $\mathrm{H}$ & 8.679516 & 4.042602 & 4.171532 \\
\hline $\mathrm{C}$ & 7.938929 & -4.62852 & 5.569092 & C & 8.051714 & -4.32767 & 5.577787 \\
\hline $\mathrm{C}$ & 8.107547 & -5.23433 & 6.998129 & $\mathrm{C}$ & 8.212462 & -4.91572 & 7.015077 \\
\hline $\mathrm{C}$ & 7.999326 & -5.79655 & 4.542673 & $\mathrm{C}$ & 8.264269 & -5.48481 & 4.558615 \\
\hline $\mathrm{C}$ & 9.119195 & -3.64724 & 5.273108 & $\mathrm{C}$ & 9.14851 & -3.24102 & 5.331516 \\
\hline $\mathrm{H}$ & 8.146151 & -4.45245 & 7.780441 & $\mathrm{H}$ & 8.159886 & -4.13175 & 7.794724 \\
\hline $\mathrm{H}$ & 7.266203 & -5.91511 & 7.237022 & $\mathrm{H}$ & 7.419669 & -5.66099 & 7.225749 \\
\hline $\mathrm{H}$ & 9.051398 & -5.8142 & 7.055889 & $\mathrm{H}$ & 9.19664 & -5.41803 & 7.111489 \\
\hline $\mathrm{H}$ & 7.97422 & -5.43101 & 3.499276 & $\mathrm{H}$ & 8.25509 & -5.12262 & 3.513712 \\
\hline $\mathrm{H}$ & 8.946104 & -6.35769 & 4.673225 & $\mathrm{H}$ & 9.25084 & -5.95859 & 4.733737 \\
\hline $\mathrm{H}$ & 7.158763 & -6.50518 & 4.68414 & $\mathrm{H}$ & 7.485144 & -6.26629 & 4.663689 \\
\hline $\mathrm{H}$ & 10.09159 & -4.1795 & 5.324579 & $\mathrm{H}$ & 10.16283 & -3.67823 & 5.438937 \\
\hline $\mathrm{H}$ & 9.015892 & -3.21013 & 4.260217 & $\mathrm{H}$ & 9.057178 & -2.82187 & 4.309899 \\
\hline $\mathrm{H}$ & 9.141275 & -2.81504 & 6.004089 & $\mathrm{H}$ & 9.051926 & -2.4069 & 6.054066 \\
\hline $\mathrm{C}$ & 4.825053 & -1.03185 & 7.502883 & $\mathrm{C}$ & 4.528332 & -1.05904 & 7.380104 \\
\hline $\mathrm{C}$ & 5.99121 & 0.008093 & 7.529287 & $\mathrm{C}$ & 5.60595 & 0.067305 & 7.493927 \\
\hline $\mathrm{C}$ & 3.497333 & -0.25921 & 7.2562 & $\mathrm{C}$ & 3.159686 & -0.38899 & 7.067211 \\
\hline $\mathrm{C}$ & 4.727569 & -1.75004 & 8.886451 & $\mathrm{C}$ & 4.407109 & -1.81143 & 8.743488 \\
\hline $\mathrm{H}$ & 6.961459 & -0.47505 & 7.755934 & $\mathrm{H}$ & 6.593532 & -0.34279 & 7.78216 \\
\hline $\mathrm{H}$ & 6.08442 & 0.520692 & 6.554102 & $\mathrm{H}$ & 5.723964 & 0.598181 & 6.531338 \\
\hline $\mathrm{H}$ & 5.803955 & 0.774682 & 8.309662 & $\mathrm{H}$ & 5.307479 & 0.805585 & 8.266722 \\
\hline $\mathrm{H}$ & 2.620905 & -0.93797 & 7.249573 & $\mathrm{H}$ & 2.343297 & -1.13512 & 6.993686 \\
\hline $\mathrm{H}$ & 3.34324 & 0.480248 & 8.067472 & $\mathrm{H}$ & 2.898203 & 0.315373 & 7.882112 \\
\hline $\mathrm{H}$ & 3.525927 & 0.292288 & 6.296616 & $\mathrm{H}$ & 3.199053 & 0.187369 & 6.122927 \\
\hline $\mathrm{H}$ & 4.525009 & -1.01191 & 9.689837 & $\mathrm{H}$ & 4.106787 & -1.1059 & 9.54556 \\
\hline $\mathrm{H}$ & 3.907685 & -2.49607 & 8.884074 & $\mathrm{H}$ & 3.645166 & -2.61419 & 8.682268 \\
\hline $\mathrm{H}$ & 5.667059 & -2.27941 & 9.137836 & $\mathrm{H}$ & 5.367452 & -2.27626 & 9.039738 \\
\hline $\mathrm{C}$ & -7.97457 & -9.32971 & 3.933937 & $\mathrm{C}$ & -7.42376 & -9.12113 & 4.416999 \\
\hline $\mathrm{C}$ & -6.95918 & -10.211 & 4.731709 & $\mathrm{C}$ & -6.33949 & -9.94511 & 5.184563 \\
\hline $\mathrm{C}$ & -8.42712 & -8.13058 & 4.827989 & $\mathrm{C}$ & -7.69685 & -7.78811 & 5.186656 \\
\hline $\mathrm{C}$ & -9.22766 & -10.1924 & 3.603487 & $\mathrm{C}$ & -8.74121 & -9.94897 & 4.381541 \\
\hline $\mathrm{H}$ & -6.63587 & -11.0826 & 4.127778 & $\mathrm{H}$ & -6.13793 & -10.9056 & 4.669151 \\
\hline $\mathrm{H}$ & -6.05525 & -9.63012 & 5.001904 & $\mathrm{H}$ & -5.3856 & -9.38531 & 5.24722 \\
\hline $\mathrm{H}$ & -7.42262 & -10.5866 & 5.667757 & $\mathrm{H}$ & -6.68008 & -10.1666 & 6.217402 \\
\hline $\mathrm{H}$ & -9.03269 & -7.406 & 4.252406 & $\mathrm{H}$ & -8.34854 & -7.11278 & 4.601915 \\
\hline $\mathrm{H}$ & -9.03093 & -8.50062 & 5.681609 & $\mathrm{H}$ & -8.18725 & -8.00335 & 6.158012 \\
\hline $\mathrm{H}$ & -7.56284 & -7.58338 & 5.250018 & $\mathrm{H}$ & -6.76046 & -7.24038 & 5.405236 \\
\hline $\mathrm{H}$ & -9.72766 & -10.4968 & 4.544375 & $\mathrm{H}$ & -9.1105 & -10.1036 & 5.414801 \\
\hline $\mathrm{H}$ & -9.96048 & -9.62735 & 2.994407 & $\mathrm{H}$ & -9.53485 & -9.42897 & 3.80988 \\
\hline $\mathrm{H}$ & -8.96136 & -11.1182 & 3.056161 & $\mathrm{H}$ & -8.58723 & -10.9497 & 3.931456 \\
\hline $\mathrm{C}$ & -7.21049 & -9.50355 & -1.17374 & $\mathrm{C}$ & -7.4202 & -9.96163 & -0.68548 \\
\hline $\mathrm{C}$ & -8.72385 & -9.24499 & -1.46839 & $\mathrm{C}$ & -8.94303 & -9.63175 & -0.8108 \\
\hline $\mathrm{C}$ & -6.3809 & -8.87599 & -2.33078 & $\mathrm{C}$ & -6.7262 & -9.54481 & -2.01397 \\
\hline $\mathrm{C}$ & -6.93772 & -11.0414 & -1.14991 & $\mathrm{C}$ & -7.23304 & -11.5007 & -0.4937 \\
\hline $\mathrm{H}$ & -9.37562 & -9.70172 & -0.69901 & $\mathrm{H}$ & -9.50431 & -9.93066 & 0.09511 \\
\hline
\end{tabular}




\begin{tabular}{|c|c|c|c|c|c|c|c|}
\hline $\mathrm{H}$ & -8.93484 & -8.15718 & -1.49225 & $\mathrm{H}$ & -9.09478 & -8.54439 & -0.96247 \\
\hline $\mathrm{H}$ & -9.00322 & -9.67436 & -2.45254 & $\mathrm{H}$ & -9.38237 & -10.1665 & -1.67799 \\
\hline $\mathrm{H}$ & -5.29369 & -9.04224 & -2.19244 & $\mathrm{H}$ & -5.64233 & -9.77701 & -2.0001 \\
\hline $\mathrm{H}$ & -6.6739 & -9.34227 & -3.29256 & $\mathrm{H}$ & -7.1793 & -10.0997 & -2.85949 \\
\hline $\mathrm{H}$ & -6.5604 & -7.78559 & -2.41515 & $\mathrm{H}$ & -6.84981 & -8.46197 & -2.21482 \\
\hline $\mathrm{H}$ & -7.19163 & -11.4925 & -2.1313 & $\mathrm{H}$ & -7.64101 & -12.0496 & -1.36745 \\
\hline $\mathrm{H}$ & -5.86892 & -11.245 & -0.93848 & $\mathrm{H}$ & -6.15866 & -11.754 & -0.3936 \\
\hline $\mathrm{H}$ & -7.54062 & -11.5528 & -0.37469 & $\mathrm{H}$ & -7.75436 & -11.8667 & 0.411957 \\
\hline $\mathrm{C}$ & -8.41773 & -3.26861 & 4.699556 & $\mathrm{C}$ & -7.54458 & -2.88891 & 4.384713 \\
\hline $\mathrm{C}$ & -8.40479 & -4.22547 & 5.935892 & $\mathrm{C}$ & -7.4421 & -3.61549 & 5.765122 \\
\hline$C$ & -7.05475 & -2.51934 & 4.64483 & $\mathrm{C}$ & -6.162 & -2.24972 & 4.060072 \\
\hline $\mathrm{C}$ & -9.54779 & -2.20574 & 4.877508 & $\mathrm{C}$ & -8.60068 & -1.74177 & 4.480289 \\
\hline $\mathrm{H}$ & -9.37105 & -4.75428 & 6.049328 & $\mathrm{H}$ & -8.4131 & -4.05281 & 6.069705 \\
\hline $\mathrm{H}$ & -7.61112 & -4.99125 & 5.828404 & $\mathrm{H}$ & -6.69952 & -4.43713 & 5.718923 \\
\hline $\mathrm{H}$ & -8.21327 & -3.65157 & 6.865996 & $\mathrm{H}$ & -7.12307 & -2.90128 & 6.552006 \\
\hline $\mathrm{H}$ & -7.00364 & -1.82521 & 3.782155 & $\mathrm{H}$ & -6.1493 & -1.7152 & 3.0895 \\
\hline $\mathrm{H}$ & -6.92398 & -1.92041 & 5.56798 & $\mathrm{H}$ & -5.90665 & -1.51355 & 4.848185 \\
\hline $\mathrm{H}$ & -6.20081 & -3.22229 & 4.577692 & $\mathrm{H}$ & -5.35526 & -3.01043 & 4.04552 \\
\hline $\mathrm{H}$ & -9.37495 & -1.6189 & 5.802796 & $\mathrm{H}$ & -8.30545 & -1.02237 & 5.271299 \\
\hline $\mathrm{H}$ & -9.56557 & -1.50317 & 4.02214 & $\mathrm{H}$ & -8.67149 & -1.19092 & 3.52323 \\
\hline $\mathrm{H}$ & -10.5479 & -2.6738 & 4.956506 & $\mathrm{H}$ & -9.60866 & -2.1264 & 4.73089 \\
\hline $\mathrm{C}$ & -11.5466 & -6.33512 & 2.003902 & $\mathrm{C}$ & -11.0702 & -6.1983 & 2.616788 \\
\hline $\mathrm{C}$ & -12.5878 & -5.24311 & 1.593302 & $\mathrm{C}$ & -12.1305 & -5.12814 & 2.198651 \\
\hline $\mathrm{C}$ & -11.5262 & -7.44193 & 0.908959 & $\mathrm{C}$ & -11.2299 & -7.44206 & 1.694074 \\
\hline $\mathrm{C}$ & -12.0012 & -6.99228 & 3.34513 & $\mathrm{C}$ & -11.3558 & -6.64709 & 4.084854 \\
\hline $\mathrm{H}$ & -12.6555 & -4.44619 & 2.359978 & $\mathrm{H}$ & -12.0784 & -4.23449 & 2.850966 \\
\hline $\mathrm{H}$ & -12.2997 & -4.76739 & 0.635761 & $\mathrm{H}$ & -11.9626 & -4.79524 & 1.156253 \\
\hline $\mathrm{H}$ & -13.5941 & -5.69513 & 1.471908 & $\mathrm{H}$ & -13.154 & -5.55128 & 2.271115 \\
\hline $\mathrm{H}$ & -10.7474 & -8.20191 & 1.117302 & $\mathrm{H}$ & -10.455 & -8.20514 & 1.9052 \\
\hline $\mathrm{H}$ & -12.5091 & -7.95304 & 0.876029 & $\mathrm{H}$ & -12.224 & -7.90388 & 1.8583 \\
\hline $\mathrm{H}$ & -11.3364 & -7.02277 & -0.09847 & $\mathrm{H}$ & -11.1621 & -7.17109 & 0.622258 \\
\hline $\mathrm{H}$ & -12.9898 & -7.47599 & 3.208953 & $\mathrm{H}$ & -12.3695 & -7.09226 & 4.150088 \\
\hline $\mathrm{H}$ & -11.2821 & -7.76521 & 3.676101 & $\mathrm{H}$ & -10.6242 & -7.40562 & 4.421198 \\
\hline $\mathrm{H}$ & -12.1044 & -6.25079 & 4.160195 & $\mathrm{H}$ & -11.3185 & -5.79922 & 4.795261 \\
\hline $\mathrm{C}$ & -13.806 & 0.830787 & -3.05562 & $\mathrm{C}$ & -13.7444 & 0.112128 & -3.23595 \\
\hline $\mathrm{C}$ & -14.734 & 1.482398 & -1.97903 & $\mathrm{C}$ & -14.6474 & 0.752195 & -2.13181 \\
\hline $\mathrm{C}$ & -13.9281 & 1.649071 & -4.37351 & $\mathrm{C}$ & -13.9651 & 0.891096 & -4.5647 \\
\hline $\mathrm{C}$ & -14.2856 & -0.62622 & -3.34839 & $\mathrm{C}$ & -14.1739 & -1.37115 & -3.4699 \\
\hline $\mathrm{H}$ & -14.7031 & 0.916034 & -1.02769 & $\mathrm{H}$ & -14.5412 & 0.218865 & -1.16706 \\
\hline $\mathrm{H}$ & -14.4112 & 2.520525 & -1.76592 & $\mathrm{H}$ & -14.3654 & 1.810676 & -1.96647 \\
\hline $\mathrm{H}$ & -15.7846 & 1.507452 & -2.3361 & $\mathrm{H}$ & -15.7146 & 0.716048 & -2.43433 \\
\hline $\mathrm{H}$ & -13.271 & 1.240503 & -5.16729 & $\mathrm{H}$ & -13.3361 & 0.486488 & -5.3829 \\
\hline $\mathrm{H}$ & -14.9733 & 1.609454 & -4.74048 & $\mathrm{H}$ & -15.0257 & 0.804293 & -4.87425 \\
\hline $\mathrm{H}$ & -13.669 & 2.714498 & -4.22101 & $\mathrm{H}$ & -13.7378 & 1.968446 & -4.45025 \\
\hline $\mathrm{H}$ & -15.3128 & -0.61063 & -3.76673 & $\mathrm{H}$ & -15.2203 & -1.41086 & -3.83608 \\
\hline $\mathrm{H}$ & -13.6181 & -1.1189 & -4.08338 & $\mathrm{H}$ & -13.522 & -1.85364 & -4.22532 \\
\hline 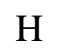 & -14.3064 & -1.25009 & -2.43441 & $\mathrm{H}$ & -14.1202 & -1.96987 & -2.5408 \\
\hline & -10.3817 & -1.05317 & 0.2822 & $\mathrm{C}$ & -10.0841 & -1.50463 & -0.000 \\
\hline
\end{tabular}




\begin{tabular}{|c|c|c|c|c|c|c|c|}
\hline $\mathrm{C}$ & -10.3959 & -2.48571 & -0.34487 & $\mathrm{C}$ & -10.1619 & -2.97159 & -0.53652 \\
\hline $\mathrm{C}$ & -8.9701 & -0.81294 & 0.894168 & $\mathrm{C}$ & -8.61923 & -1.24035 & 0.45675 \\
\hline $\mathrm{C}$ & -11.4302 & -0.98691 & 1.436988 & $\mathrm{C}$ & -11.0201 & -1.35604 & 1.23963 \\
\hline $\mathrm{H}$ & -11.3912 & -2.73027 & -0.76533 & $\mathrm{H}$ & -11.193 & -3.23697 & -0.84319 \\
\hline $\mathrm{H}$ & -9.65463 & -2.55963 & -1.16619 & $\mathrm{H}$ & -9.50401 & -3.09822 & -1.42023 \\
\hline $\mathrm{H}$ & -10.1431 & -3.24507 & 0.419967 & $\mathrm{H}$ & -9.83422 & -3.68336 & 0.244348 \\
\hline $\mathrm{H}$ & -8.89556 & 0.182791 & 1.375686 & $\mathrm{H}$ & -8.48427 & -0.22348 & 0.875847 \\
\hline $\mathrm{H}$ & -8.77099 & -1.58374 & 1.662907 & $\mathrm{H}$ & -8.34528 & -1.96748 & 1.242491 \\
\hline $\mathrm{H}$ & -8.16616 & -0.89303 & 0.135661 & $\mathrm{H}$ & -7.88989 & -1.36461 & -0.36756 \\
\hline $\mathrm{H}$ & -11.1853 & -1.74083 & 2.211191 & $\mathrm{H}$ & -10.7048 & -2.06092 & 2.033774 \\
\hline $\mathrm{H}$ & -11.4338 & 0.015374 & 1.909559 & $\mathrm{H}$ & -10.9735 & -0.327 & 1.646372 \\
\hline $\mathrm{H}$ & -12.4557 & -1.19709 & 1.076676 & $\mathrm{H}$ & -12.076 & -1.57639 & 0.988425 \\
\hline $\mathrm{C}$ & 5.881271 & 10.7598 & 0.394988 & $\mathrm{C}$ & 4.988789 & 11.10395 & 0.869366 \\
\hline $\mathrm{C}$ & 6.349611 & 12.01318 & -0.39926 & $\mathrm{C}$ & 5.313759 & 12.46553 & 0.189531 \\
\hline $\mathrm{C}$ & 5.339874 & 11.23145 & 1.781901 & $\mathrm{C}$ & 4.339527 & 11.39005 & 2.260775 \\
\hline $\mathrm{C}$ & 7.117688 & 9.828716 & 0.616893 & $\mathrm{C}$ & 6.329611 & 10.32727 & 1.078899 \\
\hline $\mathrm{H}$ & 6.809066 & 11.73628 & -1.3689 & $\mathrm{H}$ & 5.84754 & 12.32717 & -0.77153 \\
\hline $\mathrm{H}$ & 5.512341 & 12.71151 & -0.5979 & $\mathrm{H}$ & 4.397732 & 13.05825 & -0.00293 \\
\hline $\mathrm{H}$ & 7.113677 & 12.56001 & 0.188613 & $\mathrm{H}$ & 5.970505 & 13.06273 & 0.853001 \\
\hline $\mathrm{H}$ & 5.055489 & 10.37892 & 2.425853 & $\mathrm{H}$ & 4.196434 & 10.46336 & 2.847282 \\
\hline $\mathrm{H}$ & 6.116932 & 11.8167 & 2.315276 & $\mathrm{H}$ & 4.98936 & 12.06694 & 2.852421 \\
\hline $\mathrm{H}$ & 4.443375 & 11.86798 & 1.660449 & $\mathrm{H}$ & 3.347071 & 11.86466 & 2.145738 \\
\hline $\mathrm{H}$ & 7.923146 & 10.37637 & 1.148899 & $\mathrm{H}$ & 7.029256 & 10.92297 & 1.701285 \\
\hline $\mathrm{H}$ & 6.848608 & 8.939601 & 1.219992 & $\mathrm{H}$ & 6.155859 & 9.357868 & 1.585385 \\
\hline $\mathrm{H}$ & 7.518922 & 9.472433 & -0.3529 & $\mathrm{H}$ & 6.817775 & 10.12011 & 0.105522 \\
\hline $\mathrm{C}$ & 3.045134 & 9.853624 & -3.83482 & $\mathrm{C}$ & 2.548491 & 10.10824 & -3.58042 \\
\hline $\mathrm{C}$ & 4.315596 & 9.850215 & -4.74399 & $\mathrm{C}$ & 3.868026 & 10.27907 & -4.3992 \\
\hline $\mathrm{C}$ & 2.00402 & 8.870422 & -4.44389 & $\mathrm{C}$ & 1.658692 & 9.052805 & -4.29894 \\
\hline $\mathrm{C}$ & 2.413948 & 11.28324 & -3.82684 & $\mathrm{C}$ & 1.769247 & 11.46245 & -3.55789 \\
\hline $\mathrm{H}$ & 5.073897 & 10.57527 & -4.39041 & $\mathrm{H}$ & 4.521522 & 11.05968 & -3.96391 \\
\hline $\mathrm{H}$ & 4.784457 & 8.846024 & -4.75533 & $\mathrm{H}$ & 4.440127 & 9.329994 & -4.42062 \\
\hline $\mathrm{H}$ & 4.044322 & 10.12065 & -5.78531 & $\mathrm{H}$ & 3.637132 & 10.57047 & -5.44455 \\
\hline $\mathrm{H}$ & 1.068497 & 8.847468 & -3.85026 & $\mathrm{H}$ & 0.69587 & 8.904069 & -3.77075 \\
\hline $\mathrm{H}$ & 1.745246 & 9.194507 & -5.47165 & $\mathrm{H}$ & 1.431421 & 9.398098 & -5.32722 \\
\hline $\mathrm{H}$ & 2.403029 & 7.838151 & -4.50624 & $\mathrm{H}$ & 2.16907 & 8.072052 & -4.37829 \\
\hline $\mathrm{H}$ & 2.136534 & 11.58807 & -4.85701 & $\mathrm{H}$ & 1.528682 & 11.78537 & -4.59171 \\
\hline $\mathrm{H}$ & 1.497508 & 11.3001 & -3.20355 & $\mathrm{H}$ & 0.817758 & 11.35337 & -3.00009 \\
\hline $\mathrm{H}$ & 3.114867 & 12.03824 & -3.42186 & $\mathrm{H}$ & 2.356828 & 12.26647 & -3.07481 \\
\hline $\mathrm{H}$ & 5.794796 & -7.11502 & -6.41134 & $\mathrm{H}$ & 6.61561 & -6.72085 & -6.51942 \\
\hline $\mathrm{C}$ & 6.053932 & -0.22889 & -2.27518 & $\mathrm{C}$ & 6.357427 & 0.058172 & -2.21006 \\
\hline $\mathrm{C}$ & 5.947433 & -1.33845 & -0.0509 & $\mathrm{C}$ & 6.238293 & -1.11624 & -0.02023 \\
\hline $\mathrm{C}$ & 5.340592 & -1.00656 & -1.34388 & $\mathrm{C}$ & 5.657838 & -0.78257 & -1.32449 \\
\hline $\mathrm{C}$ & 6.463221 & -3.55974 & 0.95313 & $\mathrm{C}$ & 6.841519 & -3.33206 & 0.944211 \\
\hline $\mathrm{C}$ & 6.575429 & -4.9669 & 0.544312 & $\mathrm{C}$ & 7.044095 & -4.71987 & 0.504747 \\
\hline $\mathrm{C}$ & 7.99197 & -5.35536 & 0.627799 & $\mathrm{C}$ & 8.476149 & -5.03373 & 0.628629 \\
\hline $\mathrm{C}$ & 7.235432 & -0.87337 & 0.265377 & $\mathrm{C}$ & 7.486865 & -0.5897 & 0.353277 \\
\hline $\mathrm{C}$ & 7.981538 & -0.06244 & -0.70541 & $\mathrm{C}$ & 8.219208 & 0.287445 & -0.56985 \\
\hline $\mathrm{C}$ & 7.808734 & -3.0769 & 1.281418 & $\mathrm{C}$ & 8.146415 & -2.78609 & 1.331029 \\
\hline
\end{tabular}




\begin{tabular}{|c|c|c|c|c|c|c|c|}
\hline $\mathrm{C}$ & 8.187972 & -1.76384 & 0.942195 & $\mathrm{C}$ & 8.464006 & -1.44528 & 1.039776 \\
\hline $\mathrm{C}$ & 9.523419 & -1.50311 & 0.389659 & $\mathrm{C}$ & 9.800917 & -1.09719 & 0.54169 \\
\hline $\mathrm{C}$ & 8.754343 & -4.18658 & 1.079002 & $\mathrm{C}$ & 9.157526 & -3.83776 & 1.134913 \\
\hline $\mathrm{C}$ & 10.03861 & -3.93565 & 0.554844 & $\mathrm{C}$ & 10.44267 & -3.50295 & 0.662718 \\
\hline $\mathrm{C}$ & 10.42936 & -2.56498 & 0.199903 & $\mathrm{C}$ & 10.76916 & -2.10361 & 0.356561 \\
\hline $\mathrm{C}$ & 9.395491 & -0.45123 & -0.62945 & $\mathrm{C}$ & 9.649453 & -0.02651 & -0.45467 \\
\hline $\mathrm{C}$ & 10.17282 & -0.51131 & -1.80332 & $\mathrm{C}$ & 10.46719 & -0.01351 & -1.60235 \\
\hline $\mathrm{C}$ & 11.24386 & -2.626 & -1.02162 & $\mathrm{C}$ & 11.62585 & -2.0878 & -0.83732 \\
\hline $\mathrm{C}$ & 11.11724 & -1.62288 & -2.00373 & $\mathrm{C}$ & 11.47743 & -1.06681 & -1.79767 \\
\hline $\mathrm{C}$ & 10.61197 & -4.8451 & -0.44616 & $\mathrm{C}$ & 11.09772 & -4.35243 & -0.34098 \\
\hline $\mathrm{C}$ & 11.35762 & -4.03441 & -1.42092 & $\mathrm{C}$ & 11.82946 & -3.47655 & -1.26829 \\
\hline $\mathrm{C}$ & 9.569529 & -0.18811 & -3.09986 & $\mathrm{C}$ & 9.890889 & 0.311838 & -2.91074 \\
\hline $\mathrm{C}$ & 10.1345 & -1.10364 & -4.1021 & $\mathrm{C}$ & 10.53745 & -0.54548 & -3.91522 \\
\hline $\mathrm{C}$ & 11.09638 & -1.98534 & -3.42411 & $\mathrm{C}$ & 11.52303 & -1.39176 & -3.2265 \\
\hline $\mathrm{C}$ & 7.401954 & 0.255089 & -1.95019 & $\mathrm{C}$ & 7.665178 & 0.605717 & -1.82614 \\
\hline $\mathrm{C}$ & 8.213508 & 0.188571 & -3.17479 & $\mathrm{C}$ & 8.519321 & 0.616395 & -3.02309 \\
\hline $\mathrm{C}$ & 5.552406 & -2.71066 & 0.299039 & $\mathrm{C}$ & 5.907727 & -2.51623 & 0.280641 \\
\hline $\mathrm{C}$ & 11.3408 & -4.38246 & -2.78589 & $\mathrm{C}$ & 11.87819 & -3.78814 & -2.64121 \\
\hline $\mathrm{C}$ & 11.20681 & -3.33599 & -3.81001 & $\mathrm{C}$ & 11.72113 & -2.72304 & -3.64276 \\
\hline $\mathrm{C}$ & 8.541862 & -6.23165 & -0.32896 & $\mathrm{C}$ & 9.104652 & -5.85256 & -0.33072 \\
\hline $\mathrm{C}$ & 9.88009 & -5.96849 & -0.87902 & $\mathrm{C}$ & 10.44358 & -5.5017 & -0.82716 \\
\hline $\mathrm{C}$ & 9.860364 & -6.32669 & -2.30329 & $\mathrm{C}$ & 10.49138 & -5.82215 & -2.25967 \\
\hline $\mathrm{C}$ & 10.57657 & -5.55349 & -3.23494 & $\mathrm{C}$ & 11.1938 & -4.98561 & -3.14561 \\
\hline $\mathrm{C}$ & 9.428268 & -3.02075 & -5.53097 & $\mathrm{C}$ & 9.986368 & -2.45815 & -5.41648 \\
\hline $\mathrm{C}$ & 7.903198 & -1.22373 & -5.20843 & $\mathrm{C}$ & 8.354754 & -0.7551 & -5.10179 \\
\hline $\mathrm{C}$ & 5.32383 & -1.73166 & -4.12744 & $\mathrm{C}$ & 5.772963 & -1.43131 & -4.12399 \\
\hline $\mathrm{C}$ & 5.867032 & -2.62499 & -5.13594 & $\mathrm{C}$ & 6.397324 & -2.26586 & -5.13573 \\
\hline $\mathrm{C}$ & 7.132037 & -2.38332 & -5.65984 & $\mathrm{C}$ & 7.66403 & -1.94218 & -5.6086 \\
\hline $\mathrm{C}$ & 8.090615 & -3.51302 & -5.86364 & $\mathrm{C}$ & 8.689358 & -3.01274 & -5.80655 \\
\hline $\mathrm{C}$ & 7.723357 & -4.81372 & -5.53484 & $\mathrm{C}$ & 8.383504 & -4.33973 & -5.5235 \\
\hline $\mathrm{C}$ & 5.251521 & -4.02876 & -4.99199 & $\mathrm{C}$ & 5.854908 & -3.7045 & -5.0496 \\
\hline $\mathrm{C}$ & 6.275323 & -5.24796 & -5.22431 & $\mathrm{C}$ & 6.952376 & -4.86043 & -5.27514 \\
\hline $\mathrm{C}$ & 4.672878 & -3.93498 & -3.56546 & $\mathrm{C}$ & 5.224293 & -3.68124 & -3.64229 \\
\hline $\mathrm{C}$ & 4.798682 & -4.92524 & -2.60129 & $\mathrm{C}$ & 5.372888 & -4.68881 & -2.69905 \\
\hline $\mathrm{C}$ & 5.753698 & -6.05529 & -2.80715 & $\mathrm{C}$ & 6.394999 & -5.76011 & -2.89947 \\
\hline $\mathrm{C}$ & 6.512443 & -6.12899 & -3.9706 & $\mathrm{C}$ & 7.195517 & -5.76072 & -4.0368 \\
\hline $\mathrm{C}$ & 4.584057 & -2.54275 & -3.15511 & $\mathrm{C}$ & 5.046798 & -2.30742 & -3.19913 \\
\hline $\mathrm{C}$ & 8.667167 & -5.67008 & -4.83831 & $\mathrm{C}$ & 9.349483 & -5.16275 & -4.8175 \\
\hline $\mathrm{C}$ & 7.920809 & -6.47965 & -3.86957 & $\mathrm{C}$ & 8.616943 & -6.03827 & -3.89618 \\
\hline $\mathrm{C}$ & 4.577257 & -2.18231 & -1.79204 & $\mathrm{C}$ & 4.974347 & -1.98472 & -1.82841 \\
\hline $\mathrm{C}$ & 6.039374 & -0.59158 & -3.70009 & $\mathrm{C}$ & 6.410444 & -0.26638 & -3.64321 \\
\hline $\mathrm{C}$ & 9.972073 & -5.22351 & -4.53405 & $\mathrm{C}$ & 10.61623 & -4.65377 & -4.45593 \\
\hline $\mathrm{C}$ & 8.509461 & -6.80902 & -2.62774 & $\mathrm{C}$ & 9.180685 & -6.36836 & -2.6431 \\
\hline $\mathrm{C}$ & 4.820638 & -4.57395 & -1.18084 & $\mathrm{C}$ & 5.329203 & -4.37512 & -1.27047 \\
\hline $\mathrm{C}$ & 6.347217 & -6.36802 & -1.50644 & $\mathrm{C}$ & 6.960919 & -6.07582 & -1.58708 \\
\hline $\mathrm{C}$ & 7.365128 & -0.33246 & -4.25337 & $\mathrm{C}$ & 7.737243 & 0.079724 & -4.14353 \\
\hline $\mathrm{C}$ & 10.3619 & -3.86258 & -4.88814 & $\mathrm{C}$ & 10.9431 & -3.26522 & -4.76269 \\
\hline $\mathrm{C}$ & 7.70192 & -6.75347 & -1.41383 & $\mathrm{C}$ & 8.33124 & -6.38943 & -1.45714 \\
\hline
\end{tabular}




\begin{tabular}{|c|c|c|c|c|c|c|c|}
\hline $\mathrm{C}$ & 4.704267 & -3.22589 & -0.77728 & $\mathrm{C}$ & 5.125093 & -3.04715 & -0.83686 \\
\hline $\mathrm{C}$ & 9.316244 & -1.61347 & -5.12819 & $\mathrm{C}$ & 9.783655 & -1.07032 & -4.98204 \\
\hline $\mathrm{C}$ & 5.774394 & -5.45995 & -0.50415 & $\mathrm{C}$ & 6.307031 & -5.22628 & -0.58325 \\
\hline $\mathrm{C}$ & 4.122499 & -4.29052 & -6.14686 & $\mathrm{C}$ & 4.787532 & -3.99461 & -6.25387 \\
\hline $\mathrm{N}$ & 4.164716 & -5.73102 & -6.46238 & $\mathrm{~N}$ & 4.91752 & -5.4228 & -6.60166 \\
\hline $\mathrm{C}$ & 5.603559 & -6.02859 & -6.45739 & $\mathrm{C}$ & 6.368447 & -5.64516 & -6.54941 \\
\hline $\mathrm{C}$ & 3.343996 & -6.6769 & -5.67094 & $\mathrm{C}$ & 4.118202 & -6.42751 & -5.86269 \\
\hline $\mathrm{H}$ & 4.536609 & -3.78518 & -7.04328 & $\mathrm{H}$ & 5.211079 & -3.44625 & -7.12004 \\
\hline $\mathrm{H}$ & 6.060107 & -5.62971 & -7.38628 & $\mathrm{H}$ & 6.836394 & -5.20123 & -7.45179 \\
\hline $\mathrm{H}$ & 3.53629 & -6.66343 & -4.57422 & $\mathrm{H}$ & 4.272864 & -6.4338 & -4.75997 \\
\hline $\mathrm{H}$ & 3.556504 & -7.69729 & -6.04169 & $\mathrm{H}$ & 4.391994 & -7.42651 & -6.2511 \\
\hline $\mathrm{C}$ & 2.817858 & -3.58508 & -5.79354 & $\mathrm{C}$ & 3.428919 & -3.37377 & -5.94911 \\
\hline $\mathrm{C}$ & 2.571685 & -2.32714 & -6.39204 & $\mathrm{C}$ & 3.110293 & -2.14502 & -6.57344 \\
\hline $\mathrm{C}$ & 1.569701 & -1.46438 & -5.91122 & $\mathrm{C}$ & 2.021873 & -1.36149 & -6.14751 \\
\hline $\mathrm{C}$ & 0.774211 & -1.87145 & -4.82316 & $\mathrm{C}$ & 1.215432 & -1.82091 & -5.08886 \\
\hline $\mathrm{C}$ & 0.947165 & -3.15312 & -4.26159 & $\mathrm{C}$ & 1.467451 & -3.07756 & -4.5011 \\
\hline $\mathrm{C}$ & 1.971743 & -3.98913 & -4.73053 & $\mathrm{C}$ & 2.573804 & -3.83399 & -4.91657 \\
\hline $\mathrm{H}$ & 3.219885 & -1.9865 & -7.21111 & $\mathrm{H}$ & 3.762189 & -1.76286 & -7.37103 \\
\hline $\mathrm{H}$ & 1.445595 & -0.46146 & -6.33957 & $\mathrm{H}$ & 1.833681 & -0.37712 & -6.59519 \\
\hline $\mathrm{H}$ & 0.299301 & -3.48098 & -3.43862 & $\mathrm{H}$ & 0.813489 & -3.44671 & -3.70069 \\
\hline $\mathrm{H}$ & 2.27337 & -6.47521 & -5.85312 & $\mathrm{H}$ & 3.045746 & -6.2715 & -6.07604 \\
\hline $\mathrm{H}$ & 2.129325 & -4.94382 & -4.22284 & $\mathrm{H}$ & 2.784605 & -4.76972 & -4.39273 \\
\hline $\mathrm{N}$ & -0.16784 & -0.96717 & -4.22258 & $\mathrm{~N}$ & 0.176726 & -0.99189 & -4.5406 \\
\hline $\mathrm{C}$ & -1.04002 & -0.06958 & -4.85955 & $\mathrm{C}$ & -0.76738 & -0.20731 & -5.2231 \\
\hline $\mathrm{C}$ & -1.69279 & 0.607436 & -3.84468 & $\mathrm{C}$ & -1.51117 & 0.424037 & -4.24055 \\
\hline $\mathrm{N}$ & -1.23229 & 0.153081 & -2.60855 & $\mathrm{~N}$ & -1.03935 & 0.055142 & -2.97987 \\
\hline $\mathrm{C}$ & -0.32391 & -0.78492 & -2.86056 & $\mathrm{C}$ & -0.03311 & -0.78861 & -3.18815 \\
\hline $\mathrm{H}$ & -2.46501 & 1.375756 & -3.91386 & $\mathrm{H}$ & -2.3578 & 1.104871 & -4.35112 \\
\hline $\mathrm{H}$ & -1.12864 & -0.03313 & -5.94573 & $\mathrm{H}$ & -0.83172 & -0.20962 & -6.31172 \\
\hline $\mathrm{H}$ & 0.262553 & -1.32621 & -2.11656 & $\mathrm{H}$ & 0.588049 & -1.25084 & -2.41911 \\
\hline & & & & $\mathrm{O}$ & -2.67757 & 0.760074 & 1.224677 \\
\hline & & & & $\mathrm{C}$ & -3.82569 & 0.400391 & 1.749878 \\
\hline & & & & $\mathrm{O}$ & -4.72909 & -0.22925 & 1.163533 \\
\hline & & & & $\mathrm{C}$ & -3.98694 & 0.803198 & 3.23361 \\
\hline & & & & $\mathrm{H}$ & -3.54061 & 1.798853 & 3.416062 \\
\hline & & & & $\mathrm{H}$ & -5.05347 & 0.79518 & 3.52171 \\
\hline & & & & $\mathrm{H}$ & -3.44352 & 0.069713 & 3.864578 \\
\hline
\end{tabular}


Table S9. Electrochemical redox potentials (versus the saturated calomel electrode) of investigated compounds in dichloromethane

\begin{tabular}{|l|c|c|}
\hline Compound & \multicolumn{2}{|c|}{ Potential (V vs SCE) } \\
\hline & Oxidation & Reduction \\
\hline ImC60 & $-1.53,-1.02,-0.60$ & $-0.66,-1.03,-1.60$ \\
\hline $\mathbf{1}$ & $1.61,0.69,-0.23,-0.73$ & $0.88,0.13,-0.48$ \\
\hline $\mathbf{1 :} \mathbf{I m C}_{60}$ & $-1.46,-0.79,-0.65,-0.29,1.10$ & $-0.52,-0.69,-1.07,-1.55$ \\
\hline $\mathbf{2}$ & 1.03 & 0.84 \\
\hline $\mathbf{2 :} \mathbf{I m C}_{60}$ & $-1.50,-1.02,-0.60,1.33$ & $-0.69,-1.09,-1.64$ \\
\hline
\end{tabular}

Fig. S14. Fluorescence spectra $\left(\lambda_{\text {exc }}=395 \mathrm{~nm}\right)$ of $2\left(\mathrm{C}_{2}=4.4 \times 10^{-6} \mathrm{~mol} \mathrm{~L}^{-1}\right), \operatorname{ImC}_{60}\left(\mathrm{C}_{\operatorname{ImC60}}=\right.$ $\left.7.8 \times 10^{-5} \mathrm{~mol} \mathrm{~L}^{-1}\right)$ and dyad, 2:ImC $\operatorname{Imo}_{6}\left(\mathrm{C}_{2}=4.4 \times 10^{-6} \mathrm{~mol} \mathrm{~L}^{-1}, \mathrm{C}_{\mathrm{ImC60}}=1.41 \times 10^{-4} \mathrm{~mol} \mathrm{~L}^{-1}\right)$.

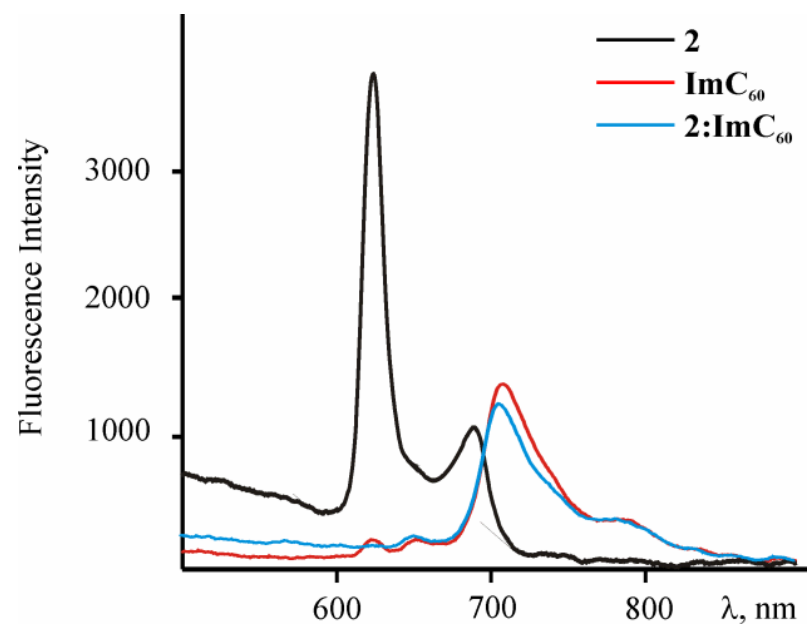

Fig. S15. Femtosecond transient spectra of 1 at the excitation wavelength of $\lambda=690 \mathrm{~nm}$ in nitrogen saturated toluene.

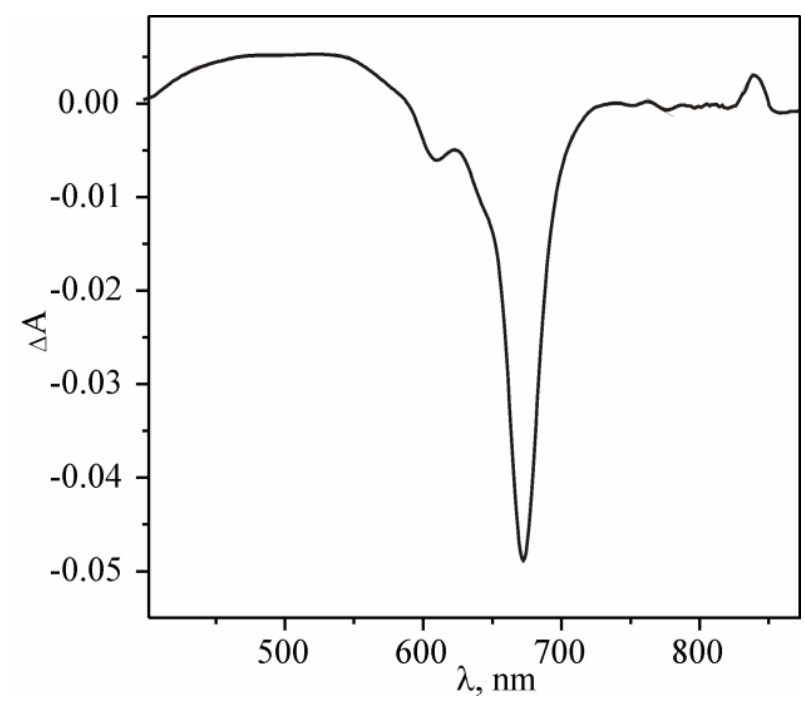


Fig. S16. Femtosecond transient spectra of 2 at the excitation wavelength of $\lambda=735 \mathrm{~nm}$ in nitrogen saturated toluene.

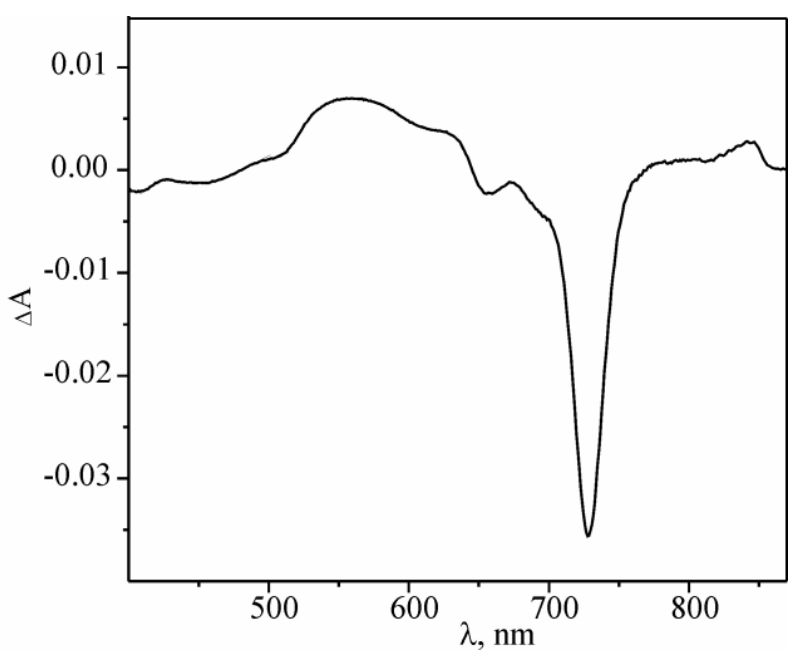

